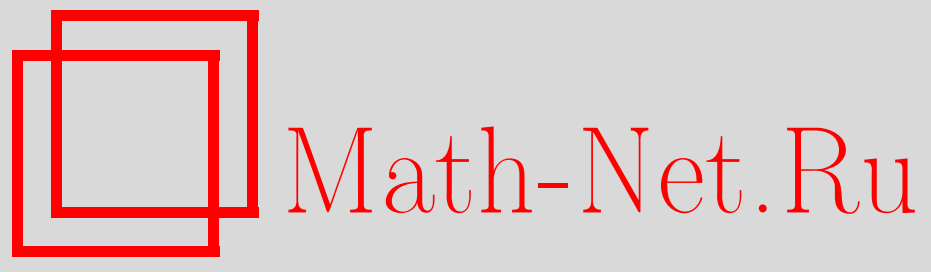

В. Г. Звягин, С. К. Кондратьев, Аттракторы уравнений неньютоновской гидродинамики, УМH, 2014, том 69, выпуск $5,81-156$

DOI: https://doi.org/10.4213/rm9615

Использование Общероссийского математического портала Math-Net.Ru подразумевает, что вы прочитали и согласны с пользовательским соглашением http://www . mathnet.ru/rus/agreement

Параметры загрузки:

IP : 54.172 .240 .79

26 апреля 2023 г., 09:58:01

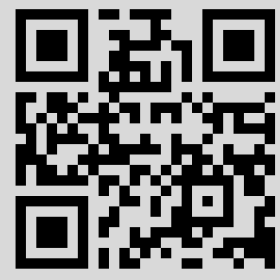




\title{
Аттракторы уравнений неньютоновской гидродинамики
}

\author{
В. Г. Звягин, С. К. Кондратьев
}

В данном обзоре излагается вариант метода траекторных аттракторов, который применяется для исследования предельного асимптотического поведения решений уравнений неньютоновской гидродинамики. Метод траекторных аттракторов возник в работах российских математиков М. И. Вишика, В.В. Чепыжова и американского математика Дж. Селла при условии инвариантности соответствующих траекторных пространств относительно полугруппы трансляций. Необходимость такого подхода была вызвана тем, что для многих уравнений математической физики, для которых начальная задача Коши имеет глобальное (слабое) решение по времени, единственность этого решения либо не установлена, либо не имеет места. В частности, такая ситуация имеет место для уравнений гидродинамики. В то же время для многих уравнений неньютоновской гидродинамики не удается построить траекторных пространств, инвариантных относительно полугруппы трансляций. В связи с этим в работах В. Г. Звягина и Д. А. Воротникова была предложена другая конструкция построения траекторных аттракторов для диссипативных систем, не использующая инвариантность траекторных пространств относительно полугруппы трансляций и основанная на топологической лемме Шуры-Буры. В работе приводятся примеры уравнений неньютоновской гидродинамики (система Джеффриса, описывающая движение земной коры, модель движения слабоконцентрированных водных растворов полимеров, система с памятью), для которых с помощью приведенной конструкции доказывается существование аттракторов как в автономном, так и в неавтономном случае. В начальной части работы также приведено краткое изложение результатов О.А. Ладыженской о существовании аттракторов двумерной системы Навье-Стокса и результатов М. И. Вишика и В. В. Чепыжова для случая аттракторов трехмерной системы Навье-Стокса.

Библиография: 34 названия.

Ключевые слова: траекторные пространства, траекторные и глобальные аттракторы автономных систем, равномерные аттракторы неавтономных систем.

DOI: $10.4213 / \mathrm{rm} 9615$

Работа выполнена при поддержке Российского научного фонда (грант № 14-21-00066).

(C) В. Г. ЗвяГин, С. К. КондРАтьев, 2014 


\section{СоДЕРЖАНИЕ}

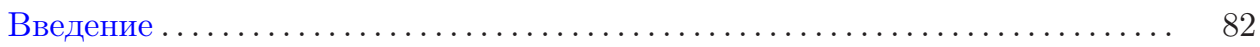

1. Глобальный аттрактор двумерной системы Навье-Стокса . . . . . . . . . . . 87

1.1. Глобальный аттрактор динамической системы ............. 87

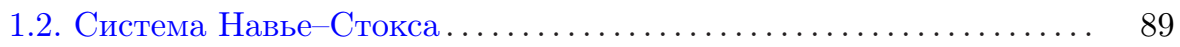

1.3. Динамическая система, порожденная двумерной системой На-

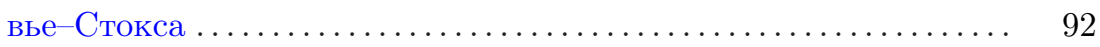

2. Теория аттракторов инвариантных пространств траекторий ........ 94

2.1. Функциональные пространства. Полугруппа трансляций....... 94

2.2. Аттракторы инвариантного пространства траекторий........ 96

2.3. Связь глобальных аттракторов полугруппы и траекторного пространства................................ 99

3. Аттракторы трехмерной системы Навье-Стокса................... 101

3.1. Аттракторы Вишика-Чепыжова .......................... 101

3.2. Аттрактор Селла................................. 106

4. Аттракторы неинвариантного пространства траекторий............. 109

4.1. Инвариантность и неинвариантность ...................... 109

4.2. Основные определения . . . . . . . . . . . . . . . . . . . . . 110

4.3. Теоремы о существовании аттракторов ................. 112

5. Траекторные и глобальные аттракторы ряда задач гидродинамики... 118

5.1. Аппроксимационно-топологический подход в задачах гидродинамики . . . . . . . . . . . . . . . . . . . . . . . . . . 118

5.2. Аттракторы системы Джеффриса с полной производной....... 119

5.3. Аттракторы модели движения слабоконцентрированных водных растворов полимеров ........................ 135

5.4. Аттракторы регуляризованной системы с памятью........... 138

6. Равномерные аттракторы неавтономных систем ................ 141

6.1. Равномерные аттракторы семейства траекторных пространств . . 141

6.2. Равномерные аттракторы системы Джеффриса с объективной

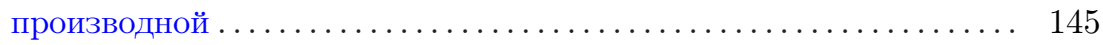

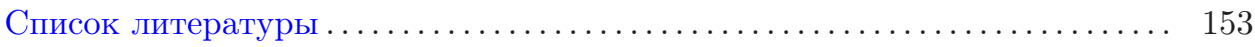

\section{Введение}

Эволюционные уравнения математической физики и, в частности, гидродинамики можно истолковывать как законы эволюции некоторых систем. Математическое описание системы включает в себя некоторое множество, называемое фазовым пространством, элементы которого отождествляются с состояниями системы, и закон эволюции, который показывает, как состояние меняется со временем. Часто для описания эволюции используется полугруппа операторов, действующих на фазовом пространстве, но это не единственный способ.

При изучении динамики систем особый интерес представляет их предельное поведение, когда время стремится к бесконечности. На практике часто 
встречаются системы со следующим свойством: "на бесконечности" их динамика сосредотачивается на небольшой части фазового пространства, называемой аттрактором. Каким бы ни было начальное состояние системы, со временем оно "забывается", и состояние системы неограниченно приближается к аттрактору. В случае таких систем естественно изучать динамику именно на аттракторах, поскольку состояния, не принадлежащие аттракторам, заведомо "преходящи". Это причина того, что изучение существования и свойств аттракторов актуально для математических проблем современного естествознания и, в частности, гидродинамики.

Понятие "аттрактор" возникло в теории динамических систем, которая изначально была тесно связана с теорией обыкновенных дифференциальных уравнений. В этой теории динамика систем обычно описывается с помощью полугруппы эволюционных операторов. Пусть $E$ - некоторое множество. Семейство отображений $\left\{S_{t}: E \rightarrow E\right\}_{t \geqslant 0}$ называется полугруппой, если выполнены следующие условия:

$$
S_{t} S_{\tau}=S_{t+\tau} \quad(t, \tau \geqslant 0) ; \quad S_{0}=\mathrm{id} .
$$

Обычно множество $E$ снабжено топологией, и тогда от операторов полугруппы требуют некоторые топологические свойства: например, непрерывность оператора $S_{t}: E \rightarrow E$ при каждом $t \geqslant 0$ и непрерывность отображений $t \mapsto S_{t} u$ для любого $u \in E$ по $t \geqslant 0$. Такая полугруппа называется полупотоком. По своему смыслу $S_{t} x$ обозначает состояние системы спустя время $t$ после того, как она находилась в состоянии $x$. Чтобы динамика системы допускала описание с помощью полугруппы, необходимо, чтобы закон эволюции был детерминированным, не менялся со временем и не обладал памятью.

Поясним, каким образом динамические системы возникают при изучении эволюционных уравнений. Пусть имеется некоторое автономное уравнение

$$
v^{\prime}=A(v) \quad(v \in E)
$$

(автономность означает, что оператор $A$ не зависит от времени). Предположим, что для каждого $v_{0} \in E$ существует единственное решение $v: \mathbb{R}_{+} \rightarrow E$ уравнения $(1)$ с начальным условием $v(0)=v_{0}$ (здесь и далее $\mathbb{R}_{+}=[0,+\infty)$ ). Тогда можно определить операторы сдвига по траекториям уравнения (1), положив $S_{t} v_{0}=v(t)(t \geqslant 0)$. С учетом автономности уравнения полугрупповое свойство для семейства операторов $\left\{S_{t}\right\}$ проверяется тривиально, а из непрерывности решений уравнения и непрерывной их зависимости от начальных данных следует, что это полупоток.

Отметим, что в случае уравнений математической физики фазовое пространство обычно бесконечномерно.

Теория бесконечномерных динамических систем и их аттракторов для уравнений с частными производными была построена во второй половине XX в. в работах О.А. Ладыженской, А. В. Бабина, М. И. Вишика, Р. Темама и других математиков. Сформулируем одно из возможных определений аттрактора. Пусть $E$ - полное метрическое пространство и $S_{t}: E \rightarrow E(t \geqslant 0)$ - динамическая система (полупоток). Замкнутое и ограниченное множество $A \subset E$ 
называется глобальным аттрактором динамической системы $\left\{S_{t}\right\}$, если оно инвариантно, т. е. $S_{t}(A)=A(t \geqslant 0)$, и притягивает ограниченные множества, т. е. образ $S_{t}(B)$ любого ограниченного множества $B \subset E$ содержится в произвольно малой окрестности аттрактора $A$ при больших значениях $t$. Имеются теоремы о существовании и свойствах глобальных аттракторов (см. п. 1.1).

Классическим приложением теории динамических систем в задачах гидродинамики является доказательство О. А. Ладыженской существования глобального аттрактора двумерной системы Навье-Стокса в работе [1] (см. также обзорную работу [2]). Этот подход оказался продуктивен и в некоторых других задачах гидродинамики.

Как было отмечено выше, для применения теории аттракторов динамических систем требуется однозначная глобальная разрешимость задачи с заданным начальным условием. Это приводит к существенным трудностям при исследовании многих задач гидродинамики. Для большого числа моделей гидродинамики не доказана глобальная разрешимость в сильном смысле, и, таким образом, сильные решения не могут быть использованы для изучения долговременной динамики. При этом может быть установлена глобальная разрешимость в слабом смысле. Но, как правило, единственность слабого решения с заданным начальным условием также не удается установить. Соответственно, нельзя корректно определить полугруппу операторов сдвига по траекториям.

Все эти трудности в полной мере обнаруживаются уже в случае трехмерной системы Навье-Стокса: для нее не установлено ни существования глобального сильного решения, ни единственности слабого.

Для построения аттракторов трехмерной системы Навье-Стокса в работах М.И. Вишика и В. В. Чепыжова [3], [4] был предложен подход, основанный на рассмотрении траекторных пространств и траекторных аттракторов. Независимо близкий метод был предложен Дж. Селлом в работе [5]. Основная идея этих теорий состоит в том, чтобы рассматривать слабые решения на $\mathbb{R}_{+}$ как точки некоторого множества $\mathscr{H}^{+}$, содержащегося в пространстве функций времени со значениями в банаховом пространстве. На этом множестве рассматривается полугруппа трансляций $\{\mathrm{T}(t)\}_{t \geqslant 0}$, действующих по правилу

$$
\mathrm{T}(t) g(s)=g(s+t)
$$

(таким образом, применение оператора $\mathrm{T}(t)$ к функции, определенной на $\mathbb{R}_{+}$, приводит к "забыванию" функцией своего поведения на отрезке $[0, t])$. Ясно, что полугруппа трансляций на самом деле действует на пространстве $\mathscr{H}^{+}$, если оно трансляционно инвариантно, т. е. $\mathrm{T}(t) \mathscr{H}^{+} \subset \mathscr{H}^{+}$при $t \geqslant 0$. В таком случае к полугруппе $\{\mathrm{T}(t)\}$ можно применять методы теории динамических систем. Получающиеся при этом аттракторы состоят из функций, на которые со временем становятся "похожи" слабые решения. Конструкции Вишика-Чепыжова и Селла нашли применения в различных задачах математической физики (см. [6]-[8]).

Подход М.И. Вишика и В.В. Чепыжова развивает идею так называемого $\left(E, E_{0}\right)$-аттрактора [9]. В новой конструкции аттракторов М. И. Вишик и 
В. В. Чепыжов рассматривают некоторое множество слабых решений $\mathscr{H}^{+}$, называемое пространством траекторий в классе функций $C\left(\mathbb{R}_{+} ; E_{0}\right) \cap L_{\infty}\left(\mathbb{R}_{+} ; E\right)$, где $E \subset E_{0}$ - надлежащим образом выбранные банаховы пространства. Элементы пространства траекторий называются траекториями. Они выбираются из всего множества слабых решений на основе энергетической оценки, обеспечивающей трансляционную инвариантность пространства траекторий. Для полугруппы трансляций, действующей на $\mathscr{H}^{+}$, методами, аналогичными методам теории динамических систем, доказывается абстрактная теорема о существовании траекторного аттрактора. Этот аттрактор притягивает множества траекторий, ограниченные в норме банахова пространства $L_{\infty}\left(\mathbb{R}_{+} ; E\right)$, причем притяжение осуществляется в топологии пространства $C\left(\mathbb{R}_{+} ; E_{0}\right)$. Траекторный аттрактор компактен в $C\left(\mathbb{R}_{+} ; E_{0}\right)$ и ограничен в $L_{\infty}\left(\mathbb{R}_{+} ; E\right)$, а также строго трансляционно инвариантен, т. е. операторы $\mathrm{T}(t)$ отображают его на себя.

Аттракторы Вишика-Чепыжова (и Селла) обладают обычными свойствами аттракторов динамических систем. В частности, они состоят из таких слабых решений, которые допускают продолжение на $\mathbb{R}$ в рассматриваемом классе решений.

Кроме траекторных аттракторов М.И. Вишик и В.В. Чепыжов рассматривают так называемые глобальные аттракторы. Такой аттрактор является множеством в пространстве $E$, которое можно рассматривать как фазовое пространство исходной задачи. Глобальный аттрактор определяется как наименьшее по включению множество, которое компактно в $E_{0}$, ограничено в $E$ и притягивает в топологии пространства $E_{0}$ множества траекторий, ограниченные в норме $E$.

Таким образом, предложен новый способ описания динамики на множестве $E$ : не с помощью задания полугруппы, а с помощью задания пространства траекторий. Отдельные траектории при этом представляют конкретные сценарии развития системы. С точки зрения описания динамики пространство траекторий является более гибким инструментом, чем полугруппа операторов. В частности, пространство траекторий позволяет обойти требование детерминированности: не запрещается наличие двух сценариев развития с одним начальным условием. В принципе, пространства траекторий могут не быть связанными с какими-либо уравнениями.

В этой теории существует замечательная связь между траекторными и глобальными аттракторами: если существует траекторный аттрактор, то в любой момент времени его сечение является глобальным аттрактором (т. е. глобальный аттрактор является множеством значений в произвольный момент времени всех функций, принадлежащих траекторному аттрактору).

В настоящем обзоре рассматривается развитие теории траекторных аттракторов для уравнений, описывающих неньютоновские среды. Обзор же всех работ по теории "инвариантных" траекторных аттракторов мы в настоящей статье не делаем, поскольку, наряду с многочисленными работами и монографией [6], есть относительно недавний обзор М. И. Вишика и В. В.Чепыжова [7] по траекторным аттракторам и их приложениям, а также недавний обзор В. В. Чепыжова [10]. Основной целью авторов настоящего обзора было только изло- 
жение материалов, связанных с их собственными исследованиями. Если мы в начале обзора кратко излагаем работы О.А. Ладыженской об аттракторах двумерного Навье-Стокса и одну из наиболее элегантных работ по траекторным аттракторам Вишика и Чепыжова с приложением к системе Навье-Стокса в трехмерном случае, то делаем это только для того, чтобы указать истоки наших исследований.

Итак, подходы Вишика-Чепыжова и Селла накладывают на рассматриваемое множество решений требование трансляционной инвариантности и некие условия типа замкнутости. Однако имеются уравнения гидродинамики (в основном неньютоновской), для которых такая ситуация не выполнена.

А именно, в различных задачах неньютоновской гидродинамики условие трансляционной инвариантности пространства траекторий оказывается ограничительным. Дело в том, что такие пространства строятся на основе энергетических оценок, нахождение которых может представлять значительные технические сложности, и далеко не всегда удается найти трансляционно инвариантную оценку. В этом отношении теория Вишика-Чепыжова получила дальнейшее развитие в работах [11]-[14]. В этих работах удалось отказаться от трансляционной инвариантности пространства траекторий (а заодно и от условий типа замкнутости). При этом сама абстрактная теория построена на совершенно другом основании, ранее не применявшемся в теории аттракторов.

Это позволило расширить границы применимости теории и исследовать аттракторы ряда систем уравнений неньютоновской гидродинамики. В частности, теория аттракторов неинвариантных пространств траекторий была успешно применена к ряду задач гидродинамики: начально-краевым задачам для системы Джеффриса с полной и объективной производными, для математической модели движения слабоконцентрированных водных растворов полимеров, для регуляризованной модели движения жидких сред с памятью [13], [15], [16].

В работе [4] М. И. Вишиком и В. В. Чепыжовым теория пространств траекторий была применена и к неавтономным уравнениям. Ими была предложена теория равномерных аттракторов, обобщающая теорию аттракторов пространств траекторий. На основе теории равномерных аттракторов было доказано существование аттракторов неавтономной системы Навье-Стокса. Теория равномерных аттракторов получила дальнейшее развитие для неинвариантных пространств траекторий в работах [12], [17].

В разделе 1 настоящей работы приводятся результаты О.А. Ладыженской о существовании аттракторов динамической системы, порожденной двумерной системой Навье-Стокса.

В разделе 2 обсуждается теория аттракторов инвариантных пространств траекторий, развитая в работах М. И. Вишика и В. В. Чепыжова.

В разделе 3 рассматривается теория аттракторов трехмерной системы НавьеСтокса, основанная на результатах М. И. Вишика и В. В. Чепыжова. Кроме того, здесь кратко описывается подход Дж. Селла.

В разделе 4 излагаются основы теории аттракторов неинвариантных пространств траекторий, развитой в работах В. Г. Звягина и Д. А. Воротникова. 
В разделе 5 приводятся приложения теории аттракторов неинвариантных пространств траекторий к задачам гидродинамики: модели движения слабоконцентрированных водных растворов полимеров, регуляризованной модели движения жидких сред с памятью и системе Джеффриса с полной производной.

В разделе 6 рассматривается теория равномерных аттракторов. В качестве приложения исследуется система Джеффриса с объективной производной.

\section{1. Глобальный аттрактор двумерной системы Навье-Стокса}

1.1. Глобальный аттрактор динамической системы. Пусть $E$ - полное метрическое пространство.

ОПредЕЛЕНиЕ 1.1. Семейство отображений $S_{t}: E \rightarrow E, t \geqslant 0$, называется nолугруппой, если $S_{0}$ - тождественное отображение и

$$
S_{t} \circ S_{\tau}=S_{t+\tau}
$$

при всех $t, \tau \geqslant 0$. Если к тому же отображения $S_{t}$ непрерывны в $E$ и для каждого $u \in E$ отображение $\mathbb{R}_{+} \rightarrow E$, действующее по правилу $t \mapsto S_{t} u$, непрерывно, полугруппа $\left\{S_{t}\right\}$ называется полупотоком. Множество $E$ называется фазовым пространством полупотока, а $S_{t}$ называются эволюиионными операторами.

Полупоток является частным случаем динамической системы. В настоящей работе не рассматриваются другие типы динамических систем, поэтому слова "динамическая система" будут относиться только к полупотокам.

Центральным вопросом теории динамических систем является изучение поведения системы при $t \rightarrow \infty$. В частности, для этого используется понятие аттрактора. Рассмотрим определение, принятое О. А. Ладыженской [2].

ОПРЕДЕлЕНИЕ 1.2. Множество $A \subset E$ называется глобалъным аттрактором динамической системы $\left\{S_{t}\right\}$, если оно замкнуто и ограничено в $E$, строго инвариантно:

$$
S_{t}(A)=A \quad(t \geqslant 0)
$$

а также обладает свойством притяжения, т. е. для любого ограниченного множества $B \subset E$

$$
\lim _{t \rightarrow \infty} \sup _{x \in B} \inf _{a \in A} \rho\left(S_{t} x, a\right)=0 .
$$

ЗАмечАниЕ 1.1. Геометрически условие (1.1) означает, что полуотклонение множества $S_{t}(B)$ от множества $A$ стремится к 0 при $t \rightarrow+\infty$. Иными словами, для любого $\varepsilon>0$ множество $S_{t}(B)$ будет содержаться в $\varepsilon$-окрестности множества $A$ при всех $t \geqslant t(\varepsilon)$.

ЗАмЕчАниЕ 1.2. Несложно показать, что если глобальный аттрактор полупотока существует, он единственный. 
Чтобы сформулировать теорему о существовании глобального аттрактора, введем еще некоторые понятия.

ОПРЕДЕЛЕНИЕ 1.3. Множество $B_{0} \subset E$ называется поглощающим, если для всякого ограниченного множества $B \subset E$ найдется такое $t_{B} \geqslant 0$, что имеет место включение

$$
S_{t}(B) \subset B_{0} \quad\left(t \geqslant t_{B}\right) .
$$

ОПРеДЕЛЕНИЕ 1.4. Динамическая система $\left\{S_{t}\right\}$ называется диссипативной, если она имеет ограниченное поглощающее множество.

ОПРеДЕЛЕНиЕ 1.5. Динамическая система $\left\{S_{t}\right\}$ называется компактной, если при $t>0$ операторы $S_{t}$ компактны, т. е. для каждого ограниченного множества $B \subset E$ множество $S_{t}(B)$ относительно компактно.

Основная теорема о существовании глобального аттрактора формулируется следующим образом [2].

Теорема 1.1. Пусть динамическая система $\left\{S_{t}\right\}$ диссипативна, компактна и имеет такое ограниченное поглощающее множество $B_{0}$, что $S_{t}\left(B_{0}\right) \subset B_{0}$ для всех $t \geqslant 0$. Тогда множество

$$
A=\omega\left(B_{0}\right) \equiv \bigcap_{\tau \geqslant 0}\left[\bigcup_{t \geqslant \tau} S_{t}\left(B_{0}\right)\right]
$$

непусто, компактно и является глобальным аттрактором динамической системы (здесь квадратные скобки обозначают замыкание в $E$ ).

Приведем также следующее утверждение из [2], характеризующее структуру глобального аттрактора.

ТеОРема 1.2. Пусть динамическая система $\left\{S_{t}\right\}$ на $E$ имеет глобальныи аттрактор $A$. Тогда точка vо принадлежит аттрактору в том и только том случае, когда найдется ограниченная функиия $v: \mathbb{R} \rightarrow E$ такая, что

$$
S_{t}(v(s))=v(s+t), \quad s \in \mathbb{R}, \quad t \geqslant 0 ; \quad v(0)=v_{0} .
$$

Динамические системы тесно связаны с эволюционными уравнениями. Пусть имеется автономное эволюционное уравнение вида

$$
\partial_{t} v=A(v)
$$

рассматриваемое в некотором пространстве $E$. Автономность означает, что оператор $A$ не зависит от $t$. Предположим, что для всякого началъного условия $v_{0} \in E$ уравнение (1.3) имеет единственное решение $v:[0,+\infty) \rightarrow E$, причем решение непрерывно по $t$ и непрерывно зависит от начальных условий. Полагая $S_{t} v_{0}=v(t)$, получаем семейство операторов $S_{t}: E \rightarrow E(t \geqslant 0)$, образующее полупоток. Проверка полугруппового свойства тривиальна. Таким 
образом, изучение эволюционных уравнений тесно связано с изучением полупотоков. Если полупоток, порожденный уравнением (1.3), имеет аттрактор, последний естественно называть аттрактором уравнения (1.3). Теорема 1.2 означает в этом случае, что аттрактор является множеством значений всех ограниченных решений уравнения (1.3), определенных на $\mathbb{R}$.

В качестве модификации понятия аттрактора можно рассмотреть такую ситуацию, когда ограниченные подмножества фазового пространства притягиваются к аттрактору в топологии, отличной от топологии фазового пространства [9]. Именно, пусть $E$ - банахово пространство, а $E_{0}$ - более широкое банахово пространство такое, что вложение $E \subset E_{0}$ непрерывно. Пусть на $E$ задана полугруппа операторов $\left\{S_{t}\right\}$. Тогда множество $\mathscr{A}$ называется $\left(E, E_{0}\right)$-аттрактором, если оно компактно в $E_{0}$ и ограничено в $E$, строго инвариантно относительно полугруппы $\left\{S_{t}\right\}$ и является $\left(E, E_{0}\right)$-притягивающим для полугруппы $\left\{S_{t}\right\}$, т. е. если для любого множества $B \subset E$, ограниченного по норме пространства $E$, выполняется следующее условие притяжения:

$$
\sup _{y \in B} \inf _{x \in \mathscr{A}}\left\|S_{t} y-x\right\|_{E_{0}} \rightarrow 0 \quad(t \rightarrow \infty) .
$$

При рассмотрении двумерной системы Навье-Стокса данное понятие нам не понадобится, однако подобная идея используется в понятии аттракторов пространств траекторий. Отметим, что в приложениях ослабление топологии притяжения (по сравнению с топологией фазового пространства) может значительно облегчать доказательство существования аттракторов.

1.2. Система Навье-Стокса. В настоящем разделе рассматривается аттрактор динамической системы, порожденной автономной двумерной системой Навье-Стокса. Предварительно мы обсудим общую слабую постановку начально-краевой задачи для системы Навье-Стокса в пространстве двух и трех измерений в неавтономном случае.

Пусть $\Omega \subset \mathbb{R}^{n}(n=2,3)$ - ограниченная область с кусочно липшицевой границей. Рассмотрим начально-краевую задачу для системы Навье-Стокса:

$$
\begin{array}{rlrl}
\frac{\partial v}{\partial t}+\sum_{i=1}^{n} v_{i} \frac{\partial v}{\partial x_{i}}-\nu \Delta v+\operatorname{grad} p & =f, & & (x, t) \in \Omega \times(0,+\infty) ; \\
\operatorname{div} v=0, & & (x, t) \in \Omega \times(0,+\infty) ; \\
\left.v\right|_{\partial \Omega}=0, & & t>0 ; \\
\left.v\right|_{t=0}=a, & & x \in \Omega .
\end{array}
$$

Здесь $v(t, x)$ - неизвестное поле скоростей, скаляр $p(t, x)$ - неизвестное давление жидкости в точке $x \in \Omega$ в момент $t, f(t, x)$ - вектор плотности внешних сил, $\nu>0$ - вязкость, $a$ - начальное значение скорости, которое будет произвольным образом выбираться из некоторого функционального пространства.

Введем функциональные пространства, стандартные в математических вопросах гидродинамики. Обозначим через $\mathscr{V}$ множество гладких соленоидальных функций $\Omega \rightarrow \mathbb{R}^{n}$, носитель которых компактен и содержится в $\Omega$. Пусть 
$H$ - замыкание множества $\mathscr{V}$ в норме пространства $\left(L_{2}(\Omega)\right)^{n}$, а $V$ - замыкание множества $\mathscr{V}$ в норме соболевского пространства $\left(W_{2}^{1}(\Omega)\right)^{n}$. Пространство $H$ гильбертово со скалярным произведением

$$
(u, v)=\int_{\Omega} u(x) \cdot v(x) d x
$$

индуцированным из $\left(L_{2}(\Omega)\right)^{n}$, а пространство $V$ гильбертово со скалярным произведением

$$
((u, v))=\int_{\Omega} \nabla u: \nabla v d x \stackrel{\text { def }}{=} \int_{\Omega} \sum_{i, j=1}^{n} \frac{\partial u_{i}}{\partial x_{j}} \frac{\partial v_{i}}{\partial x_{j}} d x
$$

где двоеточие обозначает скалярное произведение матриц: $A: B=\operatorname{tr}(A B)$ для квадратных матриц $A$ и $B$ порядка $n$. Из неравенства Пуанкаре

$$
\|u\|_{L_{2}(\Omega)} \leqslant K_{0}\|\nabla u\|_{\left(L_{2}(\Omega)\right)^{n}} \quad\left(u \in \stackrel{\circ}{W}_{2}^{1}(\Omega)\right)
$$

следует положительная определенность скалярного произведения в $V$ и непрерывность вложения $V \subset H$, а также неравенство

$$
\|u\|_{H} \leqslant K_{0}\|u\|_{V} \quad(u \in V) .
$$

Вложение $V \subset H$ компактно и плотно (см. [18]). Нормы в $H$ и $V$, отвечающие написанным выше скалярным произведениям, будем обозначать соответственно $\|\cdot\|_{H}$ и $\|\cdot\|_{V}$.

Для пространств непрерывных или суммируемых функций со значениями в банаховых пространствах мы будем использовать стандартные обозначения: $C([0, T] ; E), L_{p}([0, T] ; E)$ и т. п.

Через $H^{*}$ и $V^{*}$ обозначим пространства, сопряженные к $H$ и $V$ соответственно. Значение функционала на векторе будем обозначать угловыми скобками.

По теореме Рисса гильбертово пространство $H$ можно отождествить с его сопряженным: правило, которое функции $v \in H$ ставит в соответствие линейный функционал $u \mapsto(u, v)$, является канонической линейной изометрией пространств $H$ и $H^{*}$.

Отметим, что каждая функция $h \in H$ определяет ограниченный линейный функционал на $V$ по правилу

$$
\langle h, u\rangle=(h, u) \quad(u \in V) .
$$

Так как $V$ плотно в $H$, разные векторы из $H$ определяют разные функционалы, т. е. из равенства $\left(h_{1}-h_{2}, u\right)=0$ для любого $u \in V$ следует, что $h_{1}-h_{2}=0$. Таким образом, пространство $H$ можно считать вложенным в $V^{*}$. Это вложение непрерывно и плотно.

Пусть $f \in\left(L_{2}\left(Q_{T}\right)\right)^{n}$ и $v^{0} \in H$. 
ОПРЕДЕЛЕНИЕ 1.6. Слабым, или вариащионным, решением задачи (1.4)-(1.7) называется функция $v \in L_{2}(0, T ; V)$ такая, что для любых $\varphi \in V$ равенство

$$
\frac{d}{d t} \int_{\Omega} v(t) \cdot \varphi d x+\nu((v(t), \varphi))-\sum_{i=1}^{n} \int_{\Omega} v_{i} \frac{\partial \varphi}{\partial x_{i}} d x=(f(t), \varphi)
$$

выполняется при почти всех $t \in(0, T)$ и

$$
v(0)=v_{0} .
$$

Отметим, что для функций из $L_{2}(0, T ; V)$ значение в отдельной точке интервала не определено, поэтому начальное условие (1.12) требует пояснения. В действительности из уравнения (1.11) выводится, что производная $v^{\prime}$, понимаемая в смысле распределений на $(0, T)$ со значениями в $V^{*}$, принадлежит пространству $L_{1}\left(0, T ; V^{*}\right)$. Отсюда следует, что $v \in C\left([0, T] ; V^{*}\right)$ (точнее говоря, $v$ почти всюду совпадает с функцией, непрерывной на $[0, T]$ со значениями в $\left.V^{*}\right)$. Таким образом, имеет смысл говорить о значениях функции $v$ в любой точке отрезка $[0, T]$.

Рассмотрим операторную трактовку задачи (1.4)-(1.7). Введем операторы

$$
\begin{array}{ccrl}
A: V \rightarrow V^{*}, & \langle A u, \varphi\rangle=((u, \varphi)) & (u, \varphi \in V), \\
K: V \rightarrow V^{*}, & \langle K(u), \varphi\rangle=\sum_{i, j=1}^{n} \int_{\Omega} u_{i} u_{j} \frac{\partial \varphi_{i}}{\partial x_{j}} d x & (u, \varphi \in V) .
\end{array}
$$

Показывается (см. [18], [19]), что оператор $A$ осуществляет линейную изометрию пространств $V$ и $V^{*}$, а также $L_{2}(0, T ; V)$ и $L_{2}\left(0, T ; V^{*}\right)$ при любом $T>0$, а нелинейный оператор $K$ непрерывен в пространствах $V \rightarrow V^{*}$ и действует из $L_{2}(0, T ; V)$ в $L_{1}\left(0, T ; V^{*}\right)$ при любом $T>0$.

Уравнение (1.11) эквивалентно операторному уравнению

$$
v^{\prime}+\nu A v-K(v)=f
$$

в $L_{1}\left(0, T ; V^{*}\right)$. Это значит, что функция $v \in L_{2}(0, T ; V)$ является слабым решением задачи (1.4)-(1.7) тогда и только тогда, когда $v^{\prime} \in L_{1}\left(0, T ; V^{*}\right)$ и функция $v$ удовлетворяет операторному уравнению $(1.13)$ в $L_{2}\left(0, T ; V^{*}\right)$ и начальному условию (1.12).

Все вышесказанное относится как к двумерному, так и к трехмерному случаю. Далее в настоящем разделе будет рассматриваться только случай $n=2$. Для этого случая сформулируем теорему о существовании, единственности и непрерывной зависимости от начальных условий слабых решений системы Навье-Стокса [18]-[20].

Теорема 1.3. Пусть $\Omega$ - ограниченная область в $\mathbb{R}^{2}$ с локально липиицевой гранищей. Тогда для любого $v_{0} \in H$ существует единственное слабое решение $v$ задачи (1.4)-(1.7) в классе

$$
v \in L_{2}(0, T ; V) \cap L_{\infty}(0, T ; H), \quad v^{\prime} \in L_{2}\left(0, T ; V^{*}\right) .
$$


Функиия $v$ непрерывна на отрезке $[0, T]$ со значениями в $H$, и для любого фиксированного $t \in[0, T]$ отображение $H \rightarrow H$, действующее по правилу $v_{0} \mapsto$ $v(t)$, непрерывно.

\section{3. Динамическая система, порожденная двумерной системой На-} вье-Стокса. Далее в настоящем разделе предполагается, что размерность пространства $n=2$ и плотность внешних сил $f$ не зависит от времени и принадлежит пространству $\left(L_{2}(\Omega)\right)^{n}$.

В качестве фазового пространства полупотока, порождаемого двумерной системой Навье-Стокса, рассматривается пространство $H$. Для каждого $t \geqslant 0$ определим значение эволюционного оператора $S_{t}$ на произвольном элементе $v_{0} \in H$ как значение $v(t)$ слабого решения $v$ задачи $(1.4)-(1.7)$ на отрезке $[0, t]$. В силу теоремы 1.3 оператор $S_{t}$ определен корректно для любого $t \geqslant 0$, причем ясно, что если $v$ - решение задачи (1.4)-(1.7) на отрезке $[0, T]$, то

$$
S_{t} v_{0}=v(t) \quad \forall t \in[0, T]
$$

(так как ограничение слабого решения на любой отрезок $[0, t], t \leqslant T$, является слабым решением на этом отрезке).

Выполнение полугруппового свойства для семейства операторов $\left\{S_{t}\right\}_{t \geqslant 0}$ оказывается стандартным образом. Также из теоремы 1.3 следует непрерывность операторов $S_{t}: E \rightarrow E$ и непрерывность по $t$ функций вида $t \mapsto S_{t} a, a \in H$. Таким образом, семейство $\left\{S_{t}\right\}$ образует динамическую систему на $H$.

Покажем, что обе части уравнения (1.13) можно применить к функции $v \in$ $L_{2}(0, T ; V)$. Сформулируем согласно [18] утверждение, на основании которого это возможно.

Пусть $X, Y$ - гильбертовы пространства, причем $Y$ непрерывно и плотно вложено в $X$. Если отождествить пространство $X$ с его сопряженным, имеем цепочку

$$
Y \subset X \equiv X^{*} \subset Y^{*}
$$

где оба вложения непрерывны и плотны.

ЛЕмма 1.1. Если функиия и принадлежит $L_{2}(0, T ; Y)$, а ее производная и' принадлежит $L_{2}\left(0, T ; Y^{*}\right)$, то и почти всюду равна некоторой непрерывной функиии из $[0, T]$ в $X$ и имеет место следующее равенство, которое выполняется в смысле распределений на $(0, T)$ :

$$
\frac{d}{d t}\|u\|_{X}^{2}=2\left\langle u^{\prime}, u\right\rangle
$$

В случае $n=2$ слабые решения класса (1.14) удовлетворяют уравнению (1.13) в пространстве $L_{2}\left(0, T ; V^{*}\right)[18],[19]$, поэтому можно применить лемму 1.1. Принимая во внимание известное свойство оператора $K$ (см. [18], [19]):

$$
\langle K(v), v\rangle=0
$$


из уравнения (1.13) получаем равенство

$$
\frac{1}{2} \frac{d}{d t}\|v(t)\|_{H}^{2}+\nu\|v(t)\|_{V}^{2}=\langle f, v(t)\rangle
$$

выполняющееся почти всюду на $[0, T]$.

Оценив правую часть равенства (1.17) с помощью неравенства Коши:

$$
\langle f, v(t)\rangle \leqslant\|f\|_{V^{*}}\|v(t)\|_{V} \leqslant \frac{\nu}{2}\|v(t)\|_{V}^{2}+\frac{1}{2 \nu}\|f\|_{V^{*}}^{2}
$$

и воспользовавшись неравенством (1.9), получаем неравенство

$$
\frac{d}{d t}\|v(t)\|_{H}^{2}+2 \gamma\|v(t)\|_{H}^{2} \leqslant \frac{1}{\nu}\|f\|_{V^{*}}^{2}
$$

где $\gamma=\nu /\left(2 K_{0}^{2}\right)$. Умножим обе части последнего неравенства на $e^{2 \gamma t}$ и запишем полученное неравенство в виде

$$
\frac{d}{d t}\left(e^{2 \gamma t}\|v(t)\|_{H}^{2}\right) \leqslant \frac{1}{\nu}\|f\|_{V^{*}}^{2} e^{2 \gamma t} .
$$

Заменим здесь $t$ на $s$ и проинтегрируем по $s$ от 0 до $t$, получаем

$$
e^{2 \gamma t}\|v(t)\|_{H}^{2}-\|v(0)\|_{H}^{2} \leqslant R_{0}^{2}\left(e^{2 \gamma t}-1\right),
$$

где $R_{0}=\left(K_{0} / \nu\right)\|f\|_{V^{*}}$. Из последнего неравенства следует оценка с экспонентой для всех $t \in[0, T]$ :

$$
\|v(t)\|_{H}^{2} \leqslant R_{0}^{2}+\|v(0)\|_{H}^{2} e^{-2 \gamma t} .
$$

Отметим, что $R_{0}>0, \gamma>0$ и обе постоянные не зависят от $T$.

Так как значение $T$ произвольно, неравенство (1.18) можно интерпретировать в терминах эволюционных операторов:

$$
\left\|S_{t} u\right\|_{H}^{2} \leqslant R_{0}^{2}+\|u\|_{H}^{2} e^{-\gamma t}, \quad u \in H, \quad t \geqslant 0 .
$$

Легко видеть, что из неравенства (1.19) следует диссипативность динамической системы $\left(H, S_{t}\right)$. Действительно, предположим, что нормы $\|u\|_{H}$ элементов некоторого множества $B \subset H$ ограничены числом $R$. Выберем такое $t_{B} \geqslant 0$, чтобы выполнялось неравенство

$$
R^{2} e^{-\gamma t_{B}} \leqslant 1
$$

Тогда при $t \geqslant t_{B}$ для любого элемента $u \in B$ имеем

$$
\left\|S_{t} u\right\|_{H}^{2} \leqslant R_{0}^{2}+\|u\|_{H}^{2} e^{-\gamma t} \leqslant R_{0}^{2}+\|u\|_{H}^{2} e^{-\gamma t_{B}} \leqslant R_{0}^{2}+1 .
$$

Отсюда ясно, что шар с центром в нуле и радиусом $\left(R_{0}^{2}+1\right)^{1 / 2}$ является поглощающим множеством, т. е. динамическая система диссипативна.

Чтобы воспользоваться теоремой о существовании глобального аттрактора, остается установить, что операторы $S_{t}: H \rightarrow H$ компактны при $t>0$. Для этого воспользуемся следующим свойством слабых решений [1]. 
Теорема 1.4. Пусть $f \in H$, и пусть $v$ - слабое решение системы Навъе-Стокса на $[0, T]$ в классе (1.14). Тогда для каждого $\alpha \in(0, T]$ выполнено включение $v \in C([\alpha, T] ; V)$ и при $0<\alpha \leqslant 1$ имеет место оценка

$$
\sup _{t \in[\alpha, T]}\|v(t)\|_{V}^{2} \leqslant \frac{1}{\alpha} \mathscr{M}\left(\|v(0)\|_{H}\right),
$$

где $\mathscr{M}: \mathbb{R}_{+} \rightarrow \mathbb{R}$ - непрерьвная функиия, не зависящая от $\alpha$ и $T$.

Пусть $B \subset H$ - ограниченное (в норме $H$ ) множество. Предположим сначала, что $0<t \leqslant 1$. Из теоремы 1.4 следует оценка

$$
\sup _{v \in B}\left\|S_{t} v\right\|_{V} \leqslant \frac{1}{t} \sup _{v \in B} \mathscr{M}\left(\|v\|_{H}\right) .
$$

Множество $\left\{\|v\|_{H}: v \in B\right\}$ ограничено, а функция $\mathscr{M}$ непрерывна на $\mathbb{R}_{+}$, поэтому правая часть неравенства (1.21) конечна. Следовательно, множество $S_{t}(B)$ ограничено в норме пространства $V$. Так как вложение $V \subset H$ компактно, то множество $S_{t}(B)$ относительно компактно в пространстве $H$, что и требовалось доказать.

Для $t>1$ отображение $S_{t}=S_{t-1 / 2} S_{1 / 2}$ компактно как суперпозиция компактного и непрерывного отображений.

Выполнены условия теоремы о существовании глобального аттрактора. Следовательно, имеем следующую теорему о существовании аттрактора.

Теорема 1.5. Динамическая система $\left(H, S_{t}\right)$, порожденная системой въе-Стокса, имеет глобальный аттрактор, компактный в $H$.

Исследование динамической системы, порождаемой системой Навье-Стокса, проведено О. А. Ладыженской [1]. Теорема о существовании аттрактора была впервые сформулирована в работе А. В. Бабина и М. И. Вишика [21].

\section{2. Теория аттракторов инвариантных пространств траекторий}

2.1. Функциональные пространства. Полугруппа трансляций. Рассмотрим функциональные пространства, которые будут использоваться в этом и следующем разделах для теории траекторных аттракторов.

Пусть $E, E_{0}$ - банаховы пространства, $E \subset E_{0}$ (вложение непрерывно); также считаем, что пространство $E$ рефлексивно.

Пространство $C\left(\mathbb{R}_{+} ; E_{0}\right)$ состоит из непрерывных функций, определенных на $\mathbb{R}_{+}$и принимающих значения в пространстве $E_{0}$. На этом пространстве рассматривается топология равномерной сходимости на конечных отрезках. Эта топология может быть задана метрикой. Именно, положим

$$
\|u\|_{C\left(\mathbb{R}_{+} ; E_{0}\right)}=\sum_{k=1}^{\infty} 2^{-k} \frac{\|u\|_{C\left([0, k] ; E_{0}\right)}}{1+\|u\|_{C\left([0, k] ; E_{0}\right)}}
$$

и определим расстояние между $u, v \in C\left(\mathbb{R}_{+} ; E_{0}\right)$ как $\|u-v\|_{C\left(\mathbb{R}_{+} ; E_{0}\right)}$. Получаем инвариантную относительно сдвигов метрику на $C\left(\mathbb{R}_{+} ; E_{0}\right)$, относительно 
которой это пространства полно. Отметим, что функционал $\|\cdot\|_{C\left(\mathbb{R}_{+} ; E_{0}\right)}$ не является нормой, так как равенство

$$
\|\lambda u\|_{C\left(\mathbb{R}_{+} ; E_{0}\right)}=|\lambda|\|u\|_{C\left(\mathbb{R}_{+} ; E_{0}\right)}
$$

выполнено только при $\lambda= \pm 1$.

Пространство $L_{\infty}\left(\mathbb{R}_{+} ; E\right)$ состоит из классов совпадающих почти всюду существенно ограниченных функций $u$, определенных почти всюду на $\mathbb{R}_{+}$и принимающих значения в $E$. Норма в $L_{\infty}\left(\mathbb{R}_{+} ; E\right)$ определяется формулой

$$
\|u\|_{L_{\infty}\left(\mathbb{R}_{+} ; E\right)}=\underset{t \in \mathbb{R}_{+}}{\operatorname{vraimax}}\|u(t)\|_{E} .
$$

Если $u \in L_{\infty}\left(\mathbb{R}_{+} ; E\right)$, то имеет место неравенство

$$
\|u(t)\|_{E} \leqslant\|u\|_{L_{\infty}\left(\mathbb{R}_{+} ; E\right) \quad \text { при почти всех } t \geqslant 0 .}
$$

Пространство $L_{\infty}\left(\mathbb{R}_{+} ; E\right)$, снабженное указанной нормой, является банаховым.

Главным образом нас будут интересовать функции, принадлежащие классу $C\left(\mathbb{R}_{+} ; E_{0}\right) \cap L_{\infty}\left(\mathbb{R}_{+} ; E\right)$, т. е. непрерывные функции со значениями в $E_{0}$, которые существенно ограничены со значениями в $E$ (здесь речь идет уже о настоящих функциях, а не о классах эквивалентности).

Имеет место следующая теорема [18].

Tеорема 2.1. Пусть $E$ и $E_{0}$ - два банаховых пространства таких, что $E \subset E_{0}$, причем вложение непрерывно. Если функция и принадлежит пространству $L_{\infty}(0, M ; E)$ и непрерывна как функция со значениями в $E_{0}$, то и слабо непрерьвна как функиия со значениями в $E$.

СлЕДСТвИЕ 2.1. Функиии из класса $C\left(\mathbb{R}_{+} ; E_{0}\right) \cap L_{\infty}\left(\mathbb{R}_{+} ; E\right)$ слабо непрерывны со значениями в $E$; в частности, они принимают значения в пространстве Е при всех $t \in \mathbb{R}_{+}$.

СлЕДСТВИЕ 2.2. Функиии из класса $C\left(\mathbb{R}_{+} ; E_{0}\right) \cap L_{\infty}\left(\mathbb{R}_{+} ; E\right)$ ограничены со значениями в $E$, и дл $и \in C\left(\mathbb{R}_{+} ; E_{0}\right) \cap L_{\infty}\left(\mathbb{R}_{+} ; E\right)$

$$
\|u\|_{L_{\infty}\left(\mathbb{R}_{+} ; E\right)}=\sup _{t \geqslant 0}\|u(t)\|_{E} .
$$

Для нас будут представлять интерес также пространства функций, определенных на всей числовой оси: $C\left(\mathbb{R} ; E_{0}\right), L_{\infty}(\mathbb{R} ; E), C\left(\mathbb{R} ; E_{0}\right) \cap L_{\infty}(\mathbb{R} ; E)$. Они обладают свойствами, вполне аналогичными свойствам соответствующих пространств, описанных выше.

Рассмотрим операторы сдвигов $\mathrm{T}(t)(t \geqslant 0)$, каждый из которых функции $f$ ставит в соответствие функцию $\mathrm{T}(t) f$, действующую по следующему правилу:

$$
\mathrm{T}(t) f(s)=f(s+t) .
$$

Оператор $\mathrm{T}(t)$ является линейным ограниченным на $L_{\infty}\left(\mathbb{R}_{+} ; E\right)$ и линейным непрерывным на $C\left(\mathbb{R}_{+} ; E_{0}\right)$, и существует число $C_{t}$ такое, что неравенство

$$
\|\mathrm{T}(t) u\|_{C\left(\mathbb{R}_{+} ; E_{0}\right)} \leqslant C_{t}\|u\|_{C\left(\mathbb{R}_{+} ; E_{0}\right)}
$$

выполняется при всех $u \in C\left(\mathbb{R}_{+} ; E_{0}\right)$. 
Легко видеть, что имеет место тождество $\mathrm{T}\left(t_{1}\right) \mathrm{T}\left(t_{2}\right)=\mathrm{T}\left(t_{1}+t_{2}\right)$, а также что $\mathrm{T}(0)$ - тождественный оператор; это позволяет говорить, что семейство $\{\mathrm{T}(t): t \geqslant 0\}$ является полугруппой, которая называется полугруппой трансляиий.

Ограничение на отрезок $[0, M]$ функции $f$, определенной на $\mathbb{R}_{+}$, будет обозначаться $\Pi_{M} f$. Символом $\Pi_{+}$будем обозначать сужение на $\mathbb{R}_{+}$функции $f$, определенной на $\mathbb{R}$.

2.2. Аттракторы инвариантного пространства траекторий. Как и прежде, пусть $E, E_{0}$ - два банаховых пространства, причем будем считать, что $E$ рефлексивно и непрерывно вложено в $E_{0}$.

Рассмотрим непустое семейство функций

$$
\mathscr{H}^{+} \subset C\left(\mathbb{R}_{+} ; E_{0}\right) \cap L_{\infty}\left(\mathbb{R}_{+} ; E\right) .
$$

Множество $\mathscr{H}^{+}$будем называть пространством траекторий, а его элементы траекториями. В настоящем пункте предполагается, что пространство $\mathscr{H}^{+}$ трансляционно инвариантно, т. е.

$$
\mathrm{T}(t) \mathscr{H}^{+} \subset \mathscr{H}^{+} \quad \text { при } t \geqslant 0 .
$$

В приложениях обычный способ определения пространства траекторий состоит в том, что рассматривается некоторое множество решений (в определенном смысле) эволюционного уравнения

$$
\partial_{t} u=A(u)
$$

В качестве пространства траекторий $\mathscr{H}^{+}$рассматривается некоторое множество решений уравнения $(2.1)$, принадлежащих классу $C\left(\mathbb{R}_{+} ; E_{0}\right) \cap L_{\infty}\left(\mathbb{R}_{+} ; E\right)$. При этом не важно, в каком именно смысле понимаются решения - в сильном или слабом, и каково определение решения.

Существенно, что пространство траекторий может служить для описания динамики, определяемой эволюционным уравнением, для которого нет единственности решения с данным начальным условием или единственность не установлена.

Вообще же для теории аттракторов пространства траекторий $\mathscr{H}^{+}$наличие какого-либо уравнения несущественно, и пространство траекторий в принципе может иметь другое происхождение. Пространство траекторий является более гибким способом описания динамики, чем рассмотрение полугруппы эволюционных операторов.

Следующее понятие является аналогом понятия $\left(E, E_{0}\right)$-аттрактора.

ОПРедЕЛЕНиЕ 2.1. Множество $\mathscr{A} \subset E$ называется глобалъным аттрактором (в $\left.E_{0}\right)$ для пространства траекторий $\mathscr{H}^{+}$, если

(i) $\mathscr{A}$ компактно в $E_{0}$ и ограничено в $E$;

(ii) для любого ограниченного в $L_{\infty}\left(\mathbb{R}_{+} ; E\right)$ множества траекторий $B \subset \mathscr{H}^{+}$ выполнено свойство притяжения сечений $B(t)$ к $\mathscr{A}$ при $t \rightarrow+\infty$ по норме 
пространства $E_{0}$ :

$$
\sup _{u \in B} \inf _{y \in \mathscr{A}}\|u(t)-y\|_{E_{0}} \rightarrow 0 \quad(t \rightarrow+\infty)
$$

(iii) $\mathscr{A}$ - наименьшее по включению множество, удовлетворяющее условиям (i) и (ii).

Наряду с глобальным аттрактором, состоящим из элементов пространства $E$, рассматривается понятие траекторного аттрактора, который состоит из функций $\mathbb{R}_{+} \rightarrow E$. В силу трансляционной инвариантности пространства траекторий полугруппа трансляций действует на $\mathscr{H}^{+}$. Можно сказать, что траекторный аттрактор - это аттрактор полугруппы транслящий, действующей на $\mathscr{H}^{+}$. Дадим соответствующие определения.

ОПРеДЕЛЕНИЕ 2.2. Множество $P \subset C\left(\mathbb{R}_{+} ; E_{0}\right) \cap L_{\infty}\left(\mathbb{R}_{+} ; E\right)$ называется $n р и$ тягивающим для пространства траекторий $\mathscr{H}^{+}$в топологии $C\left(\mathbb{R}_{+} ; E_{0}\right)$, если для любого ограниченного в норме $L_{\infty}\left(\mathbb{R}_{+} ; E\right)$ множества $B \subset \mathscr{H}^{+}$выполнено соотношение

$$
\sup _{u \in B} \inf _{v \in P}\|\mathrm{~T}(t) u-v\|_{C\left(\mathbb{R}_{+} ; E_{0}\right)} \rightarrow 0 \quad(t \rightarrow+\infty)
$$

или, эквивалентно, если

$$
\sup _{u \in B} \inf _{v \in P}\|\mathrm{~T}(t) u-v\|_{C\left([0, M] ; E_{0}\right)} \rightarrow 0 \quad(t \rightarrow+\infty)
$$

для любого числа $M>0$.

ЗАмечАниЕ 2.1. Легко видеть, что в левой части (2.3) величина

$$
\inf _{v \in P}\|\mathrm{~T}(t) u-v\|_{C\left(\mathbb{R}_{+} ; E_{0}\right)}
$$

представляет собой расстояние от точки $\mathrm{T}(t) u$ до множества $P$ в пространстве $C\left(\mathbb{R}_{+} ; E_{0}\right)$, а величина

$$
\sup _{u \in B} \inf _{v \in P}\|\mathrm{~T}(t) u-v\|_{C\left(\mathbb{R}_{+} ; E_{0}\right)}
$$

- полуотклонение множества $\mathrm{T}(t) B$ от множества $P$ в том же пространстве.

ОПредЕЛЕНиЕ 2.3. Непустое множество $\mathscr{U} \subset \mathscr{H}^{+}$называется траекторнъмм аттрактором в пространстве траекторий $\mathscr{H}^{+}$относительно топологии $C\left(\mathbb{R}_{+} ; E_{0}\right)$, если

(i) $\mathscr{U}$ компактно в $C\left(\mathbb{R}_{+} ; E_{0}\right)$ и ограничено в $L_{\infty}\left(\mathbb{R}_{+} ; E\right)$;

(ii) $\mathscr{U}$ строго инвариантно относительно полугруппы $\{\mathrm{T}(t)\}$, т. е.

$$
\mathrm{T}(t) \mathscr{U}=\mathscr{U} \quad \forall t \geqslant 0 ;
$$

(iii) $\mathscr{U}$ является притягивающим множеством в пространстве $\mathscr{H}^{+}$в топологии $C\left(\mathbb{R}_{+} ; E_{0}\right)$.

4 УМН, т. 69, вып. 5 
ЗАмЕчАниЕ 2.2. Глобальный и траекторный аттракторы пространства траекторий единственны.

Приведем основные результаты о существовании аттракторов инвариантного пространства траекторий [3], [4], [6].

Теорема 2.2. Пусть пространство траекторий $\mathscr{H}^{+}$трансляционно инвариантно. Предположим, что существует притягивающее множество $P$ для пространства траекторий $\mathscr{H}^{+}$такое, что $P \subset \mathscr{H}^{+}$, причем $\mathrm{P}$ компактно в $C\left(\mathbb{R}_{+} ; E_{0}\right)$ и ограничено в $L_{\infty}\left(\mathbb{R}_{+} ; E\right)$. Тогда в пространстве $\mathscr{H}^{+}$имеется траекторный аттрактор $\mathscr{U} \subset P$.

Для каждого множества функций $P \subset C\left(\mathbb{R}_{+} ; E_{0}\right) \cap L_{\infty}\left(\mathbb{R}_{+} ; E\right)$ определим его сечение $P(t) \subset E$ в момент времени $t \geqslant 0$ :

$$
P(t)=\{u(t): u \in P\}
$$

Теорема 2.3. Пусть выполнены условия теоремы 2.2. Тогда существует глобальный аттрактор (в $\left.E_{0}\right) \mathscr{A}$ для пространства траекторий $\mathscr{H}^{+}$, причем

$$
\mathscr{A}=\mathscr{U}(0),
$$

где $\mathscr{U}$ - траекторный аттрактор.

ЗАмечАниЕ 2.3. В приложениях пространство траекторий $\mathscr{H}^{+}$может быть снабжено топологией $\Theta^{+}$, более сильной, чем топология, индуцированная из $C\left(\mathbb{R}_{+} ; E_{0}\right)$. Если при этом удается построить притягивающее множество $P \subset$ $\mathscr{H}^{+}$, компактное в $\Theta^{+}$, то траекторный аттрактор будет притягивающим в топологии $\Theta^{+}$.

Оригинальное доказательство М. И. Вишика и В. В. Чепыжова [4] центральной теоремы 2.2 состоит в том, чтобы показать, что искомым аттрактором является множество

$$
\omega(P) \stackrel{\text { def }}{=} \bigcap_{\tau \geqslant 0}\left[\bigcup_{t \geqslant \tau} \mathrm{T}(t) P\right]_{C\left(\mathbb{R}_{+} ; E_{0}\right)}
$$

(здесь квадратными скобками обозначено замыкание в пространстве $C\left(\mathbb{R}_{+} ; E_{0}\right)$ ). Таким образом, существование траекторного аттрактора доказывается методом, аналогичным используемому в теории динамических систем (ср. с формулой $(1.2))$.

Для описания структуры траекторного аттрактора полезно следующее понятие.

ОПРеДЕЛЕНИЕ 2.4. Функция $u \in C\left(\mathbb{R} ; E_{0}\right) \cap L_{\infty}(\mathbb{R} ; E)$ называется полной траекторией для пространства $\mathscr{H}^{+}$, если для любого $h \in \mathbb{R}$ имеем

$$
\Pi_{+} \mathrm{T}(h) u \in \mathscr{H}^{+} .
$$

Множество полных траекторий называется ядром пространства $\mathscr{H}^{+}$и обозначается $\mathscr{K}\left(\mathscr{H}^{+}\right)$. 
Если пространство траекторий определено как множество решений на $\mathbb{R}_{+}$ некоторого уравнения, то его ядро может быть охарактеризовано как множество решений этого уравнения, которые определены и ограничены (со значениями в $E)$ на всей числовой оси.

Следующая теорема является аналогом того утверждения, что глобальный аттрактор динамической системы является объединением ограниченных траекторий (теорема 1.2).

Теорема 2.4. Пусть выполнены условия теоремы 2.2 и $\mathscr{U}$ - траекторный, а $\mathscr{A}$ - глобальный аттракторы пространства траекторий $\mathscr{H}^{+}$. Тогда имеют место равенства

$$
\mathscr{U}=\Pi_{+} \mathscr{K}\left(\mathscr{H}^{+}\right), \quad \mathscr{A}=\mathscr{K}\left(\mathscr{H}^{+}\right)(0) .
$$

При этом ядро $\mathscr{K}\left(\mathscr{H}^{+}\right)$компактно в $C\left(\mathbb{R} ; E_{0}\right)$ и ограничено в $L_{\infty}(\mathbb{R} ; E)$.

ЗАмЕчАниЕ 2.4. Из трансляционной инвариантности аттрактора $\mathscr{U}$ следует, что сечение траекторного аттрактора не зависит от времени и в любой момент совпадает с глобальным аттрактором $\mathscr{A}=\mathscr{U}(t), t \geqslant 0$. Можно сказать, что глобальный аттрактор $\mathscr{A}$ есть обгединение всех значений всех траекторий, принадлежащих траекторному аттрактору. Равенство

$$
\mathscr{A}=\mathscr{K}\left(\mathscr{H}^{+}\right)(0)
$$

означает, что через каждую точку глобального аттрактора проходит полная траектория, и, таким образом, глобальный аттрактор является объединением значений всех полных траекторий.

\section{3. Связь глобальных аттракторов полугруппы и траекторного} пространства. Предположим, что на пространстве $E$ задана полугруппа операторов $\left\{S_{t}\right\}_{t \geqslant 0}$ таких, что функция $v_{b}: \mathbb{R}_{+} \rightarrow E$, определенная формулой

$$
v_{b}(t)=S_{t}(b)
$$

принадлежит классу $C\left(\mathbb{R}_{+} ; E_{0}\right) \cap L_{\infty}\left(\mathbb{R}_{+} ; E\right)$ при каждом $b \in E$. В этом случае естественно определить пространство траекторий $\mathscr{H}^{+}$как множество функций $v_{b}$, определяемых формулой (2.8) для всевозможных $b \in E$. Легко видеть, что определенное таким образом пространство траекторий имеет следующие два свойства:

(i) для каждого $b \in E$ существует единственная функция $v \in \mathscr{H}^{+}$такая, что $v(0)=b$;

(ii) включение $\mathrm{T}(h) \mathscr{H}^{+} \subset \mathscr{H}^{+}$имеет место при всех $h \geqslant 0$.

Действительно, (i) очевидно, а (ii) следует из того, что при всех $t \geqslant 0$

$$
\mathrm{T}(h) v_{b}(t)=v_{b}(t+h)=S_{t+h} b=S_{t} S_{h} b=v_{S_{h} b}(t),
$$

т. е. $\mathrm{T}(h) v_{b}=v_{S_{h} b} \in \mathscr{H}^{+}$. 
Нетрудно убедиться в том, что условия (i) u (ii) являются и достаточными для того, чтобы пространство траекторий $\mathscr{H}^{+}$могло быть задано с помощъю nолугруппы. Для этого достаточно определить операторы $S_{t}: E \rightarrow E$ $(t \geqslant 0)$ формулой

$$
S_{t} b=v_{b}(t)
$$

где $v_{b}$ - та единственная функция из $\mathscr{H}^{+}$, которая имеет начальное значение $v_{b}(0)=b$, и проверить, что семейство $\left\{S_{t}\right\}$ является полугруппой (проверка тривиальна).

Предположим, что на пространстве $E$ задана полугруппа операторов $\left\{S_{t}\right\}_{t \geqslant 0}$, и пусть эта полугруппа определяет пространство траекторий $\mathscr{H}^{+}$так, как это описано выше. В таком случае есть два подхода к аттрактору такой системы: можно рассматривать $\left(E, E_{0}\right)$-аттрактор полугруппы $\left\{S_{t}\right\}$ (классический подход), а можно рассматривать глобальный аттрактор пространства траекторий. Эти два подхода неэквивалентны. Целью настоящего пункта является установление связей между двумя этими подходами.

ОПРЕДЕЛЕНИЕ 2.5. Полугруппа $\left\{S_{t}\right\}$ называется ограниченной в $E$, если для любого ограниченного в $E$ множества $B$ множество $\bigcup_{t \geqslant 0} S_{t}(B)$ тоже огра-
ничено в $E$.

Имеют место следующие теоремы [14].

Теорема 2.5. Пусть полугруппа $S_{t}$ ограничена. Тогда, если существует глобальный $\left(E, E_{0}\right)$-аттрактор полугруппь $\left\{S_{t}\right\}$, то он является и глобальным аттрактором пространства траекторий $\mathscr{H}^{+}$.

ТеОРема 2.6. Пусть полугруппа $\left\{S_{t}\right\}$ ограничена, и пусть существует траекторный аттрактор $\mathscr{U}$ пространства траекторий $\mathscr{H}^{+}$. Тогда глобальный аттрактор $\mathscr{A}=\mathscr{U}(0)$ пространства траекторий $\mathscr{H}^{+}$является глобальным $\left(E, E_{0}\right)$-аттрактором полугруппь $\left\{S_{t}\right\}$.

На множестве $C\left(\mathbb{R}_{+} ; E_{0}\right) \cap L_{\infty}\left(\mathbb{R}_{+} ; E\right)$ зададим топологию, объявив последовательность $\left\{v_{m}\right\}$ сходящейся к функции в $v$, если последовательность $\left\{v_{m}\right\}$ ограничена в норме пространства $L_{\infty}\left(\mathbb{R}_{+} ; E\right)$ и сходится к $v$ в топологии пространства $C\left(\mathbb{R}_{+} ; E_{0}\right)$. Замыкание в этой топологии будем обозначать квадратными скобками.

Tеорема 2.7. Пусть существует компактное в $C\left(\mathbb{R}_{+} ; E_{0}\right)$ и ограниченное в $L_{\infty}\left(\mathbb{R}_{+} ; E\right)$ притягивающее множество $P$ для пространства траекторий $\mathscr{H}^{+}$. Пусть $\mathscr{H}^{+}=\left[\mathscr{H}^{+}\right]$, и пусть полугруппа $\left\{S_{t}\right\}$ ограничена. Тогда глобальный аттрактор $\mathscr{A}$ пространства траекторий $\mathscr{H}^{+}$(существование которого следует из теоремы 2.5) является глобальным (E, $\left.E_{0}\right)$-аттрактором полугруппы $\left\{S_{t}\right\}$.

В теореме 2.7 условие замкнутости пространства траекторий можно заменить некоторым условием на полугруппу $\left\{S_{t}\right\}$. 
ОПРЕДЕЛЕНИЕ 2.6. Будем говорить, что полугруппа $\left\{S_{t}\right\}$ замкнута, если при любом $t>0$ для всякой ограниченной в $E$ последовательности $\left\{b_{m}\right\} \subset E$ такой, что

i) $b_{m} \rightarrow b_{0} \in E$ в топологии пространства $E_{0}$,

i) последовательность $\left\{S_{t} b_{m}\right\}$ сходится в $E_{0}$, имеем $\lim _{m \rightarrow \infty} S_{t} b_{m}=b_{0}$.

Лемма 2.1. Если полугруппа $\left\{S_{t}\right\}$ замкнута, mо $\left[\mathscr{H}^{+}\right]=\mathscr{H}^{+}$.

Из леммы 2.1 и теоремы 2.7 вытекает следующая теорема.

Теорема 2.8. Пусть существует компактное в $C\left(\mathbb{R}_{+} ; E_{0}\right)$ и ограниченное в $L_{\infty}\left(\mathbb{R}_{+} ; E\right)$ притягивающее множество $P$ для пространства траекторий $\mathscr{H}^{+}$. Пусть полугруппа $\left\{S_{t}\right\}$ ограничена и замкнута. Тогда глобальный аттрактор $\mathscr{A}$ (существующий по теореме 4.3) является глобальным $\left(E, E_{0}\right)$-аттрактором.

\section{3. Аттракторы трехмерной системы Навье-Стокса}

В настоящем разделе мы рассмотрим приложение описанной выше теории аттракторов инвариантных пространств траекторий к трехмерной системе Навье-Стокса. Приводимые результаты принадлежат М. И. Вишику и В. В. Чепыжову [3], [4], [22]. Для полноты приведем также аналогичные результаты Дж. Селла [5], полученные им независимо.

3.1. Аттракторы Вишика-Чепыжова. Рассмотрим $\left\{V^{\alpha}\right\}$ - шкалу пространств, порожденную оператором Стокса $A$ (см. [23; гл. $3, \S 4])$. При $\alpha \geqslant-1 / 2$ имеет место непрерывное вложение $V^{\alpha} \subset\left(W_{2}^{\alpha}(\Omega)\right)^{n}$, причем норма

$$
\|u\|_{\alpha} \stackrel{\text { def }}{=}\left\|A^{\alpha / 2} u\right\|_{L_{2}}
$$

эквивалентна норме, индуцированной на $V^{\alpha}$ из $\left(W_{2}^{\alpha}(\Omega)\right)^{n}$. В прежних обозначениях

$$
V^{0}=H, \quad V^{1}=V, \quad V^{-1}=V^{*},
$$

причем нормы $\|\cdot\|_{0}$ и $\|\cdot\|_{H},\|\cdot\|_{1}$ и $\|\cdot\|_{V},\|\cdot\|_{-1}$ и $\|\cdot\|_{V^{*}}$ совпадают.

Далее будем считать фиксированным число $\alpha \in(0,1]$. Значение будет иметь только тот факт, что $H$ вложено в $V^{-\alpha}$ компактно.

В п. 1.2 было показано, что слабая постановка начально-краевой задачи для системы Навье-Стокса сводится к операторному уравнению

$$
v^{\prime}+\nu A v-K(v)=f
$$

в пространстве $L_{1}\left(0, T ; V^{*}\right)$, снабженному начальным условием

$$
v(0)=v_{0}
$$

М.И. Вишику и В.В. Чепыжову принадлежит следующая теорема существования слабых решений, удовлетворяющих особому энергетическому неравенству [3]. 
Теорема 3.1. Для произволъных $M>0, f \in V^{*}, v_{0} \in H$ задача (3.1), (3.2) имеет по крайней мере одно слабое решение $v$ на отрезке $[0, M]$ такое, что $v$ принадлежит $L_{\infty}(0, M ; H)$ и удовлетворяет интегралъному энергетическому неравенству

$$
-\frac{1}{2} \int_{0}^{M}\|v(s)\|_{H}^{2} \psi^{\prime}(s) d s+\nu \int_{0}^{M}\|v(s)\|_{V}^{2} \psi(s) d s \leqslant \int_{0}^{M}\langle f, v(s)\rangle \psi(s) d s,
$$

выполняющемуся для любой гладкой функиии $\psi$, носитель которой содержится в интервале $(0, M)$ и которая принимает только неотрицательные значения.

ЗАмЕчАниЕ 3.1. Если бы производная $d\|v(t)\|_{H}^{2} / d t$, понимаемая в смысле распределений, была суммируемой функцией, то первое слагаемое в левой части (3.3) можно было проинтегрировать по частям и получить

$$
\frac{1}{2} \int_{0}^{M} \frac{d}{d t}\|v(t)\|_{H}^{2} \psi(s) d s+\nu \int_{0}^{M}\|v(s)\|_{V}^{2} \psi(s) d s \leqslant \int_{0}^{M}\langle f, v(s)\rangle \psi(s) d s,
$$

откуда в силу произвольности и неотрицательности $\psi$ следовало бы неравенство

$$
\frac{1}{2} \frac{d}{d t}\|v(t)\|_{H}^{2}+\nu\|v(t)\|_{V}^{2} \leqslant\langle f, v(t)\rangle
$$

В действительности же мы не можем сделать вывод о том, что распределение $d\|v(t)\|_{H}^{2} / d t$ является суммируемой функцией: для этого было бы достаточным условие $v^{\prime} \in L_{2}\left(0, M ; V^{*}\right)$, но по определению слабого решения имеем только $v^{\prime} \in L_{1}\left(0, M ; V^{*}\right)$. Поэтому для произвольного слабого решения неравенство (3.4), вообще говоря, не имеет смысла.

В работах [4], [6] теорема 3.1 доказывается методом Галёркина. Существование слабого решения, удовлетворяющего рассматриваемой энергетической оценке, можно установить и топологическими методами, следуя аппроксимационно-топологическому подходу, описанному в [19].

Для определения пространства траекторий рассматриваются пространства $E=H$ и $E_{0}=V^{-\alpha}$ (это можно сделать, поскольку пространство $H$ рефлексивно и непрерывно вложено в $\left.V^{-\alpha}\right)$.

ОПРЕДЕЛЕНИЕ 3.1. Пространство траекторий $\mathscr{H}^{+}$уравнения (3.1) состоит из функций $v \in L_{2}^{\text {loc }}\left(\mathbb{R}_{+} ; V\right) \cap L_{\infty}\left(\mathbb{R}_{+} ; H\right)$ таких, что при любом $M>0$ функция $v$ является слабым решением уравнения (3.1) на отрезке $[0, M]$, которое удовлетворяет энергетическому неравенству (3.3).

ЗАмЕчАниЕ 3.2. Если функция $v$ определена на $\mathbb{R}_{+}$, можно заменить интегрирование по отрезку $[0, M]$ интегрированием по полуоси, т. е. для функции $v \in L_{2}^{\text {loc }}\left(\mathbb{R}_{+} ; V\right) \cap L_{\infty}\left(\mathbb{R}_{+} ; H\right)$ выполнение неравенства $(3.3)$ при всех $M>0$ эквивалентно выполнению неравенства

$$
-\frac{1}{2} \int_{0}^{\infty}\|v(s)\|_{H}^{2} \psi^{\prime}(s) d s+\nu \int_{0}^{\infty}\|v(s)\|_{V}^{2} \psi(s) d s \leqslant \int_{0}^{\infty}\langle f, v(s)\rangle \psi(s) d s,
$$


которое должно быть верно для любой гладкой функции $\psi$, носитель которой компактен и содержится в интервале $(0,+\infty)$ и которая принимает только неотрицательные значения.

Для корректности определения необходимо проверить непустоту пространства траекторий и включение $\mathscr{H}^{+} \subset C\left(\mathbb{R}_{+} ; E_{0}\right) \cap L_{\infty}\left(\mathbb{R}_{+} ; E\right)$. Прежде чем перейти к этим вопросам, отметим, что построенное пространство траекторий трансляционно инвариантно. Это следует из того, что если неравенство (3.5) выполняется для функции $v$, оно выполняется и для функций $\mathrm{T}(t) v(t \geqslant 0)$.

Включение $\mathscr{H}^{+} \subset C\left(\mathbb{R}_{+} ; E_{0}\right) \cap L_{\infty}\left(\mathbb{R}_{+} ; E\right)$ доказывается на основе теоремы вложения, которую мы сейчас сформулируем.

Пусть $E, Y$ - некоторые банаховы пространства, и пусть $E \subset Y$ (вложение непрерывно). Рассмотрим пространство

$$
W_{\infty, p}=\left\{u: u \in L_{\infty}(0, M ; E), u^{\prime} \in L_{p}(0, M ; Y)\right\}
$$

где $p>1$, а производная понимается в смысле распределений $\mathscr{D}^{\prime}(0, M ; Y)$; норма в $W_{\infty, p}$ определена равенством

$$
\|u\|_{W_{\infty, p}}=\|u\|_{L_{\infty}(0, M ; E)}+\left\|u^{\prime}\right\|_{L_{p}(0, M ; Y)} .
$$

Справедливо следующее утверждение.

Лемма 3.1. Пусть $E \subset E_{0} \subset Y$ - банаховы пространства, где вложение $E \subset E_{0}$ компактно, а вложение $E_{0} \subset Y$ непрерьвно. Тогда следующее вложение компактно:

$$
W_{\infty, p} \subset C\left([0, M] ; E_{0}\right) .
$$

Доказательство можно найти, например, в [18], [19].

Мы применим лемму для $E=H, E_{0}=V^{-\alpha}, Y=V^{*}$. Пусть $v$ - некоторая траектория. Выразив в уравнении (3.1) член с производной и оценив $v^{\prime}$, можно показать, что $v^{\prime} \in L_{4 / 3}\left(0, M ; V^{*}\right)$. По лемме 3.1 получаем $v \in C\left([0, M] ; V^{-\alpha}\right)$. В силу произвольности $M$ функция $v$ непрерывна со значениями в $V^{-\alpha}$ на всей числовой оси, что и требовалось доказать.

Пространство траекторий непусто. Более того, для любой функции $v_{0} \in H$ существует траектория $v \in \mathscr{H}^{+}$такая, что $v(0)=v_{0}$. В отличие от случая динамической системы, существование решения на $\mathbb{R}_{+}$доказывается нетривиально. Для доказательства можно выбрать последовательность $M_{m} \rightarrow+\infty$ и для каждого отрезка $\left[0, M_{m}\right](m=1,2, \ldots)$ рассмотреть решение $v_{m}$ задачи $(3.1),(3.2)$ на этом отрезке, существующее по теореме 3.1 , и затем непрерывно продолжить это решение постоянной на $\mathbb{R}_{+}$до функции $\widetilde{v}_{m}$. На основании оценки, аналогичной приводимому ниже неравенству (3.6), можно сделать вывод, что без ограничения общности последовательность $\widetilde{v}_{m}$ сходится в некоторой топологии к функции, которая и является искомой траекторией.

Таким образом, пространство траекторий определено корректно.

Основной результат о существовании траекторного и глобального аттракторов для системы Навье-Стокса содержится в следующих двух теоремах. 
Теорема 3.2 (М. И. Вишик, В.В. Чепыжов [3]). Система Навъе-Cтокса имеет траекторный аттрактор $\mathscr{U} \subset \mathscr{H}^{+}$. При этом

$$
\mathscr{U}=\Pi_{+} \mathscr{K}\left(\mathscr{H}^{+}\right) .
$$

Множество $\mathscr{U}$ ограничено в $L_{\infty}\left(\mathbb{R}_{+} ; H\right)$ и компактно в $C\left(\mathbb{R}_{+} ; V^{-\alpha}\right)$, а множество $\mathscr{K}\left(\mathscr{H}^{+}\right)$ограничено в $L_{\infty}(\mathbb{R} ; H)$ и компактно в $C\left(\mathbb{R} ; V^{-\alpha}\right)$.

Теорема 3.3 (М. И. Вишик, В. В. Чепыжов [3]). Множество

$$
\mathscr{A}=\mathscr{U}(0)=\mathscr{K}\left(\mathscr{H}^{+}\right)(0)
$$

является глобальным аттрактором системы Навъе-Стокса в топологии пространства $V^{-\alpha}$. Aттрактор $\mathscr{A}$ ограничен в $H$ и компактен в $V^{-\alpha}$.

В доказательстве теоремы 3.2 важную роль играют следующие два утверждения [4].

Лемма 3.2. Для любой функиии $u \in \mathscr{H}^{+}$выполнено следующее неравенство:

$$
\begin{gathered}
\|\mathrm{T}(t) u\|_{L_{\infty}\left(\mathbb{R}_{+} ; H\right)}+\|\mathrm{T}(t) u\|_{L_{2}(0,1 ; V)}+\left\|\mathrm{T}(t) u^{\prime}\right\|_{L_{4 / 3}\left(0,1 ; V^{*}\right)} \\
\leqslant C\|u\|_{L_{\infty}(0,1 ; H)}^{2} e^{-\gamma t}+R_{0} \quad \forall t \geqslant 0
\end{gathered}
$$

где $C>0, R_{0}>0, \gamma>0 ; C, \gamma$ и $R_{0}$ не зависят от $и$.

Лемма 3.3. Пусть дана последовательность $\left\{u_{n}\right\} \subset \mathscr{H}^{+}$, ограниченная в $L_{\infty}\left(\mathbb{R}_{+} ; H\right)$. Предположим, что для некоторой функиии $u^{*} \in C\left(\mathbb{R}_{+} ; V^{-\alpha}\right)$ имеет место предельное соотношение

$$
u_{n} \rightarrow u^{*} \quad(n \rightarrow \infty) \quad \text { в } C\left(\mathbb{R}_{+} ; V^{-\alpha}\right) .
$$

Тогда $u^{*} \in \mathscr{H}^{+}$.

Приведем доказательство теоремы 3.2. Для этого рассмотрим множество

$$
\begin{aligned}
P_{0}=\{ & u \in C\left(\mathbb{R}_{+} ; V^{-\alpha}\right) \cap L_{\infty}\left(\mathbb{R}_{+} ; H\right): \\
& \left.\quad \underset{h \geqslant 0}{\operatorname{vrai} \max }\left\{\|u\|_{L_{\infty}(h, h+1 ; H)}+\left\|u^{\prime}\right\|_{L_{4 / 3}\left(h, h+1 ; V^{*}\right)}\right\} \leqslant 2 R_{0}\right\},
\end{aligned}
$$

где $R_{0}$ - постоянная из неравенства (3.6). Очевидно, что множество $P_{0}$ ограничено в $L_{\infty}\left(\mathbb{R}_{+} ; H\right)$. Кроме того, оно относительно компактно в $C\left(\mathbb{R}_{+} ; V^{-\alpha}\right)$. Это является следствием того, что при любом $M>0$ множество $\Pi_{M} P_{0}$, состоящее из ограничений функций, принадлежащих $P_{0}$, на отрезок $[0, M]$, ограничено в $L_{\infty}(0, M ; H)$, а множество производных функций из $\Pi_{M} P_{0}$ ограничено в $L_{4 / 3}\left(0, M ; V^{*}\right)$, и, следовательно, множество $\Pi_{M} P_{0}$ относительно компактно в $C\left([0, M] ; V^{-\alpha}\right)$ (лемма 3.1). Покажем, что в действительности множество $P_{0}$ компактно в $C\left(\mathbb{R}_{+} ; V^{-\alpha}\right)$, для чего требуется проверить его замкнутость. Пусть

$$
\left\{u_{n}\right\} \subset P_{0} \quad \text { и } \quad u_{n} \rightarrow u^{*} \quad \text { в } C\left(\mathbb{R}_{+} ; V^{-\alpha}\right) .
$$


Покажем, что $u^{*} \in P_{0}$. Зафиксируем $h>0$. Из определения множества $P_{0}$ следует, что последовательность $\left\{u_{n}\right\}$ ограничена в $L_{\infty}(h, h+1 ; H)$, а последовательность $\left\{u_{n}^{\prime}\right\}$ ограничена в $L_{4 / 3}\left(h, h+1 ; V^{*}\right)$, поэтому без ограничения общности можно считать, что

$$
\begin{array}{ll}
u_{n} \rightarrow u^{*} & * \text {-слабо в } L_{\infty}(h, h+1 ; H), \\
u_{n}^{\prime} \rightarrow\left(u^{*}\right)^{\prime} & \text { в смысле распределений, } \\
u_{n}^{\prime} \rightarrow\left(u^{*}\right)^{\prime} & \text { слабо в } L_{4 / 3}\left(h, h+1 ; V^{*}\right) .
\end{array}
$$

Отсюда следует оценка

$$
\begin{aligned}
& \left\|u^{*}\right\|_{L_{\infty}(h, h+1 ; H)}+\left\|\left(u^{*}\right)^{\prime}\right\|_{L_{4 / 3}\left(h, h+1 ; V^{*}\right)} \\
& \quad \leqslant \underline{n \rightarrow \infty}\left\|u_{n}\right\|_{L_{\infty}(h, h+1 ; H)}+\varliminf_{n \rightarrow \infty}\left\|u_{n}^{\prime}\right\|_{L_{4 / 3}\left(h, h+1 ; V^{*}\right)} \\
& \leqslant \underline{\lim _{n \rightarrow \infty}}\left(\left\|u_{n}\right\|_{L_{\infty}(h, h+1 ; H)}+\left\|u_{n}^{\prime}\right\|_{L_{4 / 3}\left(h, h+1 ; V^{*}\right)}\right) \leqslant 2 R_{0}
\end{aligned}
$$

для $h>0$. Таким образом, $u^{*} \in P_{0}$, и компактность множества $P_{0}$ доказана.

Покажем, что множество $P_{0} \cap \mathscr{H}^{+}$компактно в $C\left(\mathbb{R}_{+} ; V^{-\alpha}\right)$, ограничено в $L_{\infty}\left(\mathbb{R}_{+} ; H\right)$ и является притягивающим для пространства $\mathscr{H}^{+}$. Как только это будет установлено, доказываемая теорема будет следовать из теорем 2.2, 2.4.

Будучи подмножеством $P_{0}$, множество $P_{0} \cap \mathscr{H}^{+}$относительно компактно в $C\left(\mathbb{R}_{+} ; V^{-\alpha}\right)$ и ограничено в $L_{\infty}\left(\mathbb{R}_{+} ; H\right)$. Чтобы доказать его компактность, нужно убедиться в его замкнутости. Пусть

$$
\left\{u_{n}\right\} \subset P_{0} \cap \mathscr{H}^{+} \quad \text { и } \quad u_{n} \rightarrow u^{*} \quad \text { в } C\left(\mathbb{R}_{+} ; V^{-\alpha}\right) .
$$

Покажем, что $u^{*} \in P_{0} \cap \mathscr{H}^{+}$. В силу компактности множества $P_{0}$ имеем $u^{*} \in P_{0}$. Далее, последовательность $\left\{u_{n}\right\}$ содержится в $P_{0}$ и, следовательно, ограничена в $L_{\infty}\left(\mathbb{R}_{+} ; H\right)$; тогда по лемме 3.3 имеем $u^{*} \in \mathscr{H}^{+}$. Таким образом,

$$
u^{*} \in P_{0} \cap \mathscr{H}^{+},
$$

и множество $P_{0} \cap \mathscr{H}^{+}$компактно в $C\left(\mathbb{R}_{+} ; V^{-\alpha}\right)$.

Легко видеть, что множество $P_{0} \cap \mathscr{H}^{+}$является поглощающим для $\mathscr{H}^{+}$. Действительно, пусть множество $B \subset \mathscr{H}^{+}$ограничено в $L_{\infty}\left(\mathbb{R}_{+} ; H\right)$, т. е.

$$
\|u\|_{L_{\infty}\left(\mathbb{R}_{+} ; H\right)} \leqslant R \quad \text { для некоторого } R>0 \text { и всех } u \in B .
$$

Выберем число $t_{1}$ так, что

$$
R^{2} C e^{-\gamma t_{1}}+R_{0} \leqslant 2 R_{0}
$$

(здесь $C$ и $\gamma$ - постоянные из неравенства (3.6)), тогда при любом $t \geqslant t_{1}$ в силу оценки (3.6) получаем неравенство

$$
\|\mathrm{T}(t) u\|_{L_{\infty}(0,1 ; H)}+\left\|\mathrm{T}(t) u^{\prime}\right\|_{L_{4 / 3}\left(0,1 ; V^{*}\right)} \leqslant 2 R_{0} .
$$


Следовательно, $\mathrm{T}(t) u \in P_{0}$, т. е. $\mathrm{T}(t) B \subset P_{0}$ при $t \geqslant t_{1}$. В силу же инвариантности траекторного пространства имеем $\mathrm{T}(t) B \subset \mathscr{H}^{+}$при всех $t \geqslant 0$. Таким образом,

$$
\mathrm{T}(t) B \subset P_{0} \cap \mathscr{H}^{+} \text {при } t \geqslant t_{1},
$$

и множество $P_{0} \cap \mathscr{H}^{+}$действительно является поглощающим.

Мы установили, что $P_{0} \cap \mathscr{H}^{+}-$поглощающее (а значит, и притягивающее) множество, компактное в $C\left(\mathbb{R}_{+} ; V^{-\alpha}\right)$ и ограниченное в $L_{\infty}\left(\mathbb{R}_{+} ; H\right)$. Это доказывает теорему 3.2 .

ЗАмечание 3.3. Следуя оригинальным рассуждениям М.И. Вишика и В.В. Чепыжова [4], в доказательстве теоремы 3.2 можно было бы сразу потребовать, чтобы в множество $P_{0}$ входили только функции из $\mathscr{H}^{+}$. Мы не делаем так, чтобы аналогия с доказательствами соответствующих теорем для рассматриваемых ниже моделей была более выраженной.

ЗАмЕчАНИЕ 3.4. Из доказательства теоремы 3.2 и теоремы 2.2 следует, что выполнено включение $\mathscr{U} \subset P_{0} \cap \mathscr{H}^{+}$.

3.2. Аттрактор Селла. Кратко опишем результаты Дж. Селла об аттракторах трехмерной системы Навье-Стокса [5].

Основой подхода Дж. Селла является рассмотрение действия полугруппы трансляций на некотором множестве слабых решений системы Навье-Стокса и нахождение аттрактора этой полугруппы. Таким образом, “глобальный аттрактор" в смысле терминологии Селла является "траекторным аттрактором" в смысле Вишика-Чепыжова. Селл не рассматривает аттракторов в пространствах функций, не зависящих от времени.

Введем необходимые функциональные пространства. Пусть $X$ - банахово пространство с нормой $\|\cdot\|_{X}$.

Через $L_{\infty}(a, b ; X)(-\infty \leqslant a<b \leqslant \infty)$ обозначается банахово пространство существенно ограниченных функций $u:(0, \infty) \rightarrow X$ с обычной нормой

$$
\|u\|_{L_{\infty}(a, b ; X)}=\underset{a<s<b}{\operatorname{vraimax}}\|u(s)\| .
$$

Пусть $1 \leqslant p<\infty$. Через $L_{p}^{\text {loc }}(0, \infty ; H)$ обозначается пространство функций $u:(0, \infty) \rightarrow X$ таких, что

$$
\int_{\tau}^{T}\|u(s)\|_{X}^{p} d s<\infty
$$

для всех $0<\tau \leqslant T<\infty$. Это пространство Фреше, топология которого порождается семейством псевдонорм

$$
N_{n}(u)=\left(\int_{n^{-1}}^{n}\|u(s)\|_{X}^{p} d s\right)^{1 / p}, \quad n=2,3, \ldots
$$


Через $L_{p}^{\text {loc }}[0, \infty ; H)$ обозначается пространство функций $u:(0, \infty) \rightarrow X$, для которых интегралы

$$
\int_{0}^{T}\|u(s)\|_{X}^{p} d s
$$

конечны при $0<T<\infty$. Это также пространство Фреше, в котором топология порождается семейством псевдонорм

$$
\widetilde{N}_{n}(u)=\left(\int_{n}^{n+1}\|u(s)\|_{X}^{p} d s\right)^{1 / p}, \quad n=0,1,2, \ldots
$$

Все эти пространства являются метризуемыми локально выпуклыми топологическими векторными пространствами, и они полны.

Напомним, что в топологическом векторном пространстве множество $B$ называется ограниченным, если для всякой окрестности нуля $U$ найдется такое $r>0$, что $B \subset r U$. В пространстве $L_{p}^{\text {loc }}(0, \infty ; H)$ ограниченность множества $B$ означает, что

$$
\sup \left\{N_{n}(u): u \in B, n=2,3, \ldots\right\}<\infty,
$$

где $\left\{N_{n}\right\}$ - семейство псевдонорм $(3.7)$; в пространстве $L_{p}^{\text {loc }}[0, \infty ; H)$ ограниченность множества $B$ означает, что

$$
\sup \left\{\widetilde{N}_{n}(u): u \in B, n=0,1, \ldots\right\}<\infty,
$$

где $\left\{\widetilde{N}_{n}\right\}$ - семейство псевдонорм (3.8).

Аналог теоремы 1.1 верен и для динамических систем, фазовое пространство которых - замкнутое множество в полном топологическом векторном пространстве, если в определении аттрактора, диссипативности и компактности полупотока ограниченность понимать в вышеуказанном смысле.

Основным пространством, в котором происходят рассмотрения Селла, является пространство Фреше $L_{2}^{\text {loc }}[0, \infty ; H)$. На этом пространстве действует полугруппа трансляций $\{\mathrm{T}(t)\}$. Подход Селла заключается в том, чтобы рассмотреть эту полугруппу на некотором замкнутом положительно инвариантном подмножестве пространства $L_{2}^{\text {loc }}[0, \infty ; H)$ и исследовать ее методами теории аттракторов динамических систем.

Слабые решения системы Навье-Стокса понимаются в следующем смысле.

Пусть $\lambda_{1}$ - наименьшее собственное значение оператора Стокса, и пусть $c_{0}=$ $\left(\nu \lambda_{1}\right)^{-1}$.

ОПРЕДЕЛЕНИЕ 3.2. Функция $v$ называется слабым решением класса ЛереХопфа системы Навье-Стокса с правой частью $f \in L_{\infty}\left(0, \infty ;\left(L_{2}(\Omega)\right)^{3}\right)$, если выполнены следующие условия:

1) $v \in L_{\infty}(0, \infty ; H) \cap L_{2}^{\text {loc }}[0, \infty ; V)$;

2) $v^{\prime} \in L_{4 / 3}^{\text {loc }}\left[0, \infty ; V^{*}\right)$

3) при почти всех $t$ и почти всех $t_{0}$ таких, что $t>t_{0}>0$, выполняются неравенства

$$
\|v(t)\|_{H}^{2} \leqslant e^{-\nu \lambda_{1}\left(t-t_{0}\right)}\left\|v\left(t_{0}\right)\right\|_{H}^{2}+c_{0}^{2}\|f\|_{L_{\infty}\left(0, \infty ;\left(L_{2}(\Omega)\right)^{3}\right)}
$$


и

$$
\|v(t)\|_{H}^{2}+2 \nu \int_{t_{0}}^{t}\|v(s)\|_{V}^{2} d s \leqslant\left\|v\left(t_{0}\right)\right\|_{H}^{2}+2 \int_{t_{0}}^{t}(f(s), v(s)) d s
$$

4) при всех $t \geqslant t_{0} \geqslant 0$ выполняется тождество

$$
\begin{aligned}
\left\langle v(t)-v\left(t_{0}\right), \varphi\right\rangle & +\nu \int_{t_{0}}^{t}((v(s), \varphi)) d s \\
& +\int_{t_{0}}^{t}\langle K(v(s)), \varphi\rangle d s=\int_{t_{0}}^{t}(f(s), v(s)) d s
\end{aligned}
$$

для всех $\varphi \in V$.

Множество слабых решений класса Лере-Хопфа системы Навье-Стокса с правой частью $f$ обозначается $W_{\mathrm{LH}}(f)$.

Следует отметить, что для любого слабого решения $v$ имеем $v \in L_{2}^{\text {loc }}[0, \infty ; H)$, и так как $v \in L_{\infty}(0, \infty ; H)$, то $v \in C_{w}([0, \infty) ; H)$ (по теореме 2.1 ), поэтому значения слабого решения принадлежат $H$ при каждом $t$.

В [5] существование слабых решений класса Лере-Хопфа начально-краевой задачи для системы Навье-Стокса устанавливается методом Галёркина.

Множество $W_{\mathrm{LH}}$ является подмножеством в $L_{2}^{\text {loc }}[0, \infty ; H)$, положительно инвариантным относительно полугруппы трансляций $\{\mathrm{T}(t)\}$. Однако неизвестно, является ли $W_{\mathrm{LH}}$ замкнутым подмножеством и, таким образом, является ли $W_{\mathrm{LH}}$ полным пространством. Последнее обстоятельство важно для применения теоремы о существовании аттрактора динамической системы. Чтобы получить замкнутое фазовое пространство для полугруппы трансляций, в [5] понятие слабого решения расширяется следующим образом.

ОПРЕДЕЛЕНИЕ 3.3. Функция $v$ называется обобщенным слабъм решением системы Навье-Стокса с правой частью $f \in L_{\infty}\left(0, \infty ;\left(L_{2}(\Omega)\right)^{3}\right)$, если выполнены следующие условия:

1) $v \in L_{2}^{\text {loc }}[0, \infty ; H) \cap L_{\infty}(0, \infty ; V)$ и $v \in L_{\infty}(a, \infty ; H)$ для каждого $a>0$;

2) $v^{\prime} \in L_{4 / 3}^{\text {loc }}\left(0, \infty ; V^{*}\right)$;

3) при почти всех $t$ и почти всех $t_{0}$ таких, что $t>t_{0}>0$, выполняются неравенства (3.9) и (3.10);

4) при всех $t \geqslant t_{0} \geqslant 0$ тождество (3.11) выполняется для любых $\varphi \in V$.

Множество обобщенных слабых решений системы с правой частью $f$ обозначается $W(f)$.

Из определения следует, что $W_{\mathrm{LH}}(f) \subset W(f)$ для любой функции $f \in$ $L_{\infty}\left(0, \infty ;\left(L_{2}(\Omega)\right)^{3}\right)$. В то же время $W(f) \subset L_{2}^{\text {loc }}[0, \infty ; H)$, т. е. понятие обобщенного решения не выводит из интересующего нас класса функций.

Далее рассматривается только автономный случай. Зафиксируем функцию $f \in\left(L_{2}(\Omega)\right)^{3}$ и положим $W=W(f), W_{\mathrm{LH}}=W_{\mathrm{LH}}(f)$. В [5] показано, что множество $W$ является замкнутым подмножеством $L_{2}^{\text {loc }}[0, \infty ; H)$ и, следовательно, полным пространством Фреше и что полугруппа трансляций определяет на $W$ полупоток. Глобальный аттрактор этого полупотока понимается в [5] как аттрактор системы Навье-Стокса в $\Omega$. 
Диссипативность полупотока $\{\mathrm{T}(t)\}$ на $W$ выводится из неравенства (3.9), а доказательство компактности этого полупотока существенным образом опирается на неравенство (3.10), поскольку последнее содержит член

$$
\int_{t_{0}}^{t}\|v(s)\|_{V}^{2} d s
$$

Основной результат о существовании аттрактора представлен в следующей теореме.

Tеорема 3.4 [5]. Пусть $f \in\left(L_{2}(\Omega)\right)^{3} u W=W(f), W_{\mathrm{LH}}=W_{\mathrm{LH}}(f)$. Тогда существует аттрактор $A$ системъ Навъе-Стокса в $\Omega, A \subset W_{\mathrm{LH}}$. Aттрактор A притягивает ограниченные множества в $W$.

Еще раз отметим, что построенный аттрактор Дж. Селл называет глобальным, и это понятие соответствует траекторному аттрактору в терминологии М. И. Вишика и В. В. Чепыжова.

В теореме 3.4 включение $A \subset W_{\mathrm{LH}}$ является специфическим свойством системы Навье-Стокса, а остальные утверждения являются следствием абстрактных теорем об аттракторах динамических систем. Можно показать [5], что все слабые решения, принадлежащие аттрактору $A$, являются сужениями на неотрицательную полуось слабых решений, определенных на $\mathbb{R}$.

\section{4. Аттракторы неинвариантного пространства траекторий}

4.1. Инвариантность и неинвариантность. Условие трансляционной инвариантности пространства траекторий на первый взгляд выглядит естественным: если некая функция $v$ является решением автономного уравнения, то при любом разумном определении решения функция $\mathrm{T}(t) v(t \geqslant 0)$ также должна быть решением этого уравнения. Однако в конкретных задачах условие трансляционной инвариантности оказывается ограничительным, и естественными являются как раз неинвариантные пространства. Поясним причину этого обстоятельства.

Если для некоторой модели гидродинамики не установлено глобальное существование сильных решений, естественно изучать аттракторы для слабых решений. Однако вся совокупность слабых решений той или иной задачи, как правило, малообозрима, и логически не исключено существование слабых решений с патологическими свойствами. Поэтому представляют интерес не произвольные слабые решения, а слабые решения, удовлетворяющие тем или иным энергетическим оценкам. Как мы убедились на примере трехмерной системы Навье-Стокса, разумное определение пространства траекторий включает в себя энергетическую оценку.

При исследовании как разрешимости, так и существования аттракторов той или иной задачи гидродинамики отправной точкой часто служит дифференциальное тождество или неравенство, подобное (1.17) или (3.4). Однако двумерная система Навье-Стокса оказывается скорее исключением, чем правилом, 
в том отношении, что тождество (1.17) установлено непосредственно для самих слабых решений. В более сложных системах аналогичные соотношения могут быть установлены лишь для некоторых аппроксимаций, как в случае неравенства (3.4) для трехмерной системы Навье-Стокса. При переходе к пределу это неравенство сохранилось лишь в обобщенном виде, как интегральное неравенство (3.3). В других задачах не удается установить даже такого.

Важным свойством неравенства (3.3) является его трансляционная инвариантность. Именно это обстоятельство позволяет определить инвариантное пространство траекторий. Однако фактически существование аттракторов обычно основывается на оценках, содержащих убывающие экспоненты (ср. с (1.18) в классическом случае динамической системы и с (3.6) в случае аттракторов пространства траекторий). Существенно, что такие оценки оказываются более устойчивыми по отношению к операции предельного перехода. Отметим, что в некоторых случаях экспоненциальная оценка также может быть инвариантной (как у Дж. Селла, п. 3.2).

Общая ситуация, которая ведет к неинвариантным пространствам траекторий, может быть сформулирована следующим образом: во-первых, для аппроксимаций устанавливается "дифференциальная" оценка; во-вторых, из “дифференциальной” выводится "экспоненциальная" оценка; в-третьих, доказывается существование решений исходной задачи, удовлетворяющих "экспоненциальной” оценке, и на их основе строится пространство траекторий.

4.2. Основные определения. Понятие пространства траекторий как подмножества класса функций $C\left(\mathbb{R}_{+} ; E_{0}\right) \cap L_{\infty}\left(\mathbb{R}_{+} ; E\right)$ было введено в п. 2.1. В настоящем разделе мы отказываемся от требования трансляционной инвариантности пространства траекторий и требуем только, чтобы оно было непусто.

В приложениях обычный способ определения пространства траекторий состоит в том, что рассматривается некоторое множество решений (в определенном смысле) эволюционного уравнения

$$
\partial_{t} u=A(u)
$$

принадлежащих некоторому классу $\mathscr{F} \subset C\left(\mathbb{R}_{+} ; E_{0}\right) \cap L_{\infty}\left(\mathbb{R}_{+} ; E\right)$. Как правило, если $u$ - решение уравнения, то $\mathrm{T}(h) u(h \geqslant 0)$ - также его решение, поэтому инвариантность пространства траекторий $\mathrm{T}(h) \mathscr{H}^{+} \subset \mathscr{H}^{+}$может нарушаться только в том случае, когда неинвариантен класс $\mathscr{F}$, т. е. если включение $\mathrm{T}(h) \mathscr{F} \subset \mathscr{F}$ не выполняется при некоторых $h \geqslant 0$. Это достаточно типичный случай, который имеет место, например, когда $\mathscr{F}$ состоит из функций, удовлетворяющих некоторой оценке, не сохраняющейся под действием трансляционных операторов $\mathrm{T}(h)$.

В случае неинвариантного пространства траекторий используются те же понятия глобального аттрактора и притягивающего множества, что и в случае инвариантного. Но траекторные аттракторы определяются по-другому.

ОПРеДЕЛЕНИЕ 4.1. Множество $\mathscr{A} \subset E$ называется глобалъным аттрактором (в $\left.E_{0}\right)$ пространства траекторий $\mathscr{H}^{+}$, если оно удовлетворяет следующим условиям: 
(i) множество $\mathscr{A}$ компактно в $E_{0}$ и ограничено в $E$;

(ii) для всякого ограниченного в $L_{\infty}\left(\mathbb{R}_{+} ; E\right)$ множества $B \subset \mathscr{H}^{+}$выполняется условие притяжения

$$
\sup _{u \in B} \inf _{y \in \mathscr{A}}\|u(t)-y\|_{E_{0}} \rightarrow 0 \quad(t \rightarrow \infty)
$$

(iii) множество $\mathscr{A}$ является наименьшим по включению, удовлетворяющим условиям (i) и (ii) (т. е. $\mathscr{A}$ содержится в каждом множестве, удовлетворяющем этим условиям).

ОПРедЕЛЕНИЕ 4.2. Множество $P \subset C\left(\mathbb{R}_{+} ; E_{0}\right) \cap L_{\infty}\left(\mathbb{R}_{+} ; E\right)$ называется траекторным полуаттрактором (пространства траекторий $\mathscr{H}^{+}$), если оно удовлетворяет следующим условиям:

(i) множество $P$ компактно в $C\left(\mathbb{R}_{+} ; E_{0}\right)$ и ограничено в $L_{\infty}\left(\mathbb{R}_{+} ; E\right)$;

(ii) имеет место включение $\mathrm{T}(t) P \subset P$ для всех $t \geqslant 0$;

(iii) множество $P$ является притягивающим, т. е. для любого множества $B \subset \mathscr{H}^{+}$, ограниченного в норме пространства $L_{\infty}\left(\mathbb{R}_{+} ; E\right)$, выполняется условие

$$
\lim _{t \rightarrow \infty} \sup _{v \in B} \inf _{u \in \mathscr{U}}\|\mathrm{T}(t) v-u\|_{C\left(\mathbb{R}_{+} ; E_{0}\right)}=0 .
$$

ОПРедЕЛЕНиЕ 4.3. Множество $P \subset C\left(\mathbb{R}_{+} ; E_{0}\right) \cap L_{\infty}\left(\mathbb{R}_{+} ; E\right)$ называется траекторным квазиаттрактором (пространства траекторий $\mathscr{H}^{+}$), если оно удовлетворяет условиям (i), (iii) определения 4.2 , а также условию

(ii') имеет место включение $\mathrm{T}(t) P \supset P$ для всех $t \geqslant 0$.

ОПРЕДЕЛЕНИЕ 4.4. Множество $P \subset C\left(\mathbb{R}_{+} ; E_{0}\right) \cap L_{\infty}\left(\mathbb{R}_{+} ; E\right)$ называется траекторным аттрактором (пространства траекторий $\mathscr{H}^{+}$), если оно одновременно является и траекторным полуаттрактором, и траекторным квазиаттрактором, т. е. если оно удовлетворяет условиям (i), (iii) определения 4.2, а также условию

(ii") имеет место равенство $\mathrm{T}(t) P=P$ для всех $t \geqslant 0$.

ОПРЕДЕЛЕНИЕ 4.5. Минималъным траекторным аттрактором пространства траекторий $\mathscr{H}^{+}$называется наименьший по включению траекторный аттрактор, т. е. такой траекторный аттрактор, который содержится в любом другом траекторном аттракторе.

Отметим отличия введенной терминологии от той, которая использовалась в п. 2.2. Именно, траекторный аттрактор, определенный в п. 2.2, непременно содержался в пространстве траекторий, из чего следовала его единственность, и, таким образом, дополнительных эпитетов это понятие не требовало. Определение 4.4, напротив, допускает то, что траекторный аттрактор может не содержаться в пространстве траекторий, и поэтому траекторных аттракторов, вообще говоря, много. Интерес же представляет наименьший из них (именно он будет давать в сечении глобальный аттрактор, о чем речь ниже), поэтому нужно ввести определение 4.5 минимального траекторного аттрактора. 
ЗАмЕчАниЕ 4.1. Если существует минимальный траекторный аттрактор или глобальный аттрактор, он единственный.

ЗАмЕчАНИЕ 4.2. Если траекторный аттрактор в смысле определения 4.4 содержится в пространстве траекторий, он является минимальным [14].

Целесообразно ввести понятие ядра произвольного множества в $C\left(\mathbb{R}_{+} ; E_{0}\right) \cap$ $L_{\infty}\left(\mathbb{R}_{+} ; E\right)$.

ОПРеДЕЛЕНИЕ 4.6. Ядром множества $P \subset C\left(\mathbb{R}_{+} ; E_{0}\right) \cap L_{\infty}\left(\mathbb{R}_{+} ; E\right)$ называется множество

$$
\mathscr{K}(P)=\left\{u \in C\left(\mathbb{R} ; E_{0}\right) \cap L_{\infty}(\mathbb{R} ; E): \forall t \in \mathbb{R} \Pi_{+} \mathrm{T}(t) u \in P\right\},
$$

где $\Pi_{+}$- сужение на $\mathbb{R}_{+}$функции $f$, определенной на $\mathbb{R}$.

Из определения непосредственно следует включение $\Pi_{+} \mathscr{K}(P) \subset P$.

4.3. Теоремы о существовании аттракторов. В случае неинвариантного пространства траекторий доказательство теоремы о существовании минимального траекторного аттрактора опирается не на понятие $\omega$-предельного множества, а на следующее топологическое предложение.

Лемма 4.1 (лемма Шуры-Буры). Пусть в компакте $X$ дана совокупность замкнутых множеств $\sigma=\left\{\Phi_{\alpha}\right\}$ с непустым пересечением $\Phi=\bigcap_{\alpha} \Phi_{\alpha}$. Пусть $O(\Phi)$ - произвольная окрестность множества $\Phi$. Тогда существует конечное число множеств $\Phi_{\alpha} \in \sigma$, пересечение которых лежит в $O(\Phi)$.

СлЕДСТВИЕ 4.1. Если семейство непустых замкнутых множеств $\sigma=\left\{\Phi_{\alpha}\right\}$ компакта $X$ направлено по включению (т.е. для любых $\Phi_{\alpha_{1}} \in \sigma, \Phi_{\alpha_{2}} \in \sigma$ имеется $\Phi_{\alpha_{3}} \in \sigma$ mакое, что $\left.\Phi_{\alpha_{3}} \subset \Phi_{\alpha_{1}} \cap \Phi_{\alpha_{2}}\right)$, то множество $\Phi=\bigcap_{\alpha} \Phi_{\alpha}$ непусто и каждая его окрестность содержит некоторое $\Phi_{\alpha} \in \sigma$.

Сформулируем основную теорему о существовании минимального траекторного аттрактора [13], [14].

ТеОрема 4.1. Пусть пространство траекторий $\mathscr{H}^{+}$имеет траекторный полуаттрактор $P$. Тогда существует минимальный траекторный аттрактор $\mathscr{U}$ пространства траекторий $\mathscr{H}^{+}$, имеют место соотношения

$$
\Pi_{+} \mathscr{K}\left(\mathscr{H}^{+}\right) \subset \mathscr{U}=\Pi_{+} \mathscr{K}(\mathscr{U}) \subset \Pi_{+} \mathscr{K}(P) \subset P
$$

и ядро $\mathscr{K}\left(\mathscr{H}^{+}\right)$относительно компактно в $C\left(\mathbb{R} ; E_{0}\right)$ и ограничено в $L_{\infty}(\mathbb{R} ; E)$.

ДокАЗАТЕЛЬСтво. Воспользуемся следствием 4.1: пусть $X=P$, и пусть $\left\{\Phi_{\alpha}\right\}$ - семейство всех траекторных полуаттракторов пространства траекторий $\mathscr{H}^{+}$, содержащихся в $P$. Несложно показать, что пересечение двух траекторных полуаттракторов является траекторным полуаттрактором, поэтому условия следствия 4.1 выполнены и пересечение $\Phi=\bigcap_{\alpha} \Phi_{\alpha}$ непусто. 
Покажем, что $\Phi$ является траекторным полуаттрактором. Ясно, что это множество компактно в $C\left(\mathbb{R}_{+} ; E_{0}\right)$ и ограничено в $L_{\infty}\left(\mathbb{R}_{+} ; E\right)$. Его инвариантность проверяется тривиально: при $t \geqslant 0$ имеем

$$
T(t) \Phi=\mathrm{T}(t) \bigcap_{\alpha} \Phi_{\alpha} \subset \bigcap_{\alpha} \mathrm{T}(t) \Phi_{\alpha} \subset \bigcap_{\alpha} \Phi_{\alpha}=\Phi .
$$

Остается проверить, что множество $\Phi$ является притягивающим. Пусть $B \subset$ $\mathscr{H}^{+}$- некоторое множество, ограниченное в $L_{\infty}\left(\mathbb{R}_{+} ; E\right)$. Рассмотрим произвольное число $\varepsilon>0$. По следствию 4.1 существует полуаттрактор $\Phi_{\alpha}$, содержащийся в $\varepsilon / 2$-окрестности (в норме $\left.C\left(\mathbb{R}_{+} ; E_{0}\right)\right)$ множества $\Phi$, т. е. такой, что для любого $v \in \Phi_{\alpha}$ выполняется неравенство

$$
\inf _{w \in \Phi}\|w-v\|_{C\left(\mathbb{R}_{+} ; E_{0}\right)}<\frac{\varepsilon}{2} .
$$

Поскольку $\Phi_{\alpha}-$ притягивающее множество, то найдется такое $h>0$, что для всех $t \geqslant h$ имеем

$$
\sup _{u \in B} \inf _{v \in \Phi_{\alpha}}\|\mathrm{T}(t) u-v\|_{C\left(\mathbb{R}_{+} ; E_{0}\right)}<\frac{\varepsilon}{2} .
$$

Для любых $u \in B, v \in \Phi_{\alpha}, w \in \Phi$ и $t \geqslant 0$ имеем

$$
\|\mathrm{T}(t) u-w\|_{C\left(\mathbb{R}_{+} ; E_{0}\right)} \leqslant\|\mathrm{T}(t) u-v\|_{C\left(\mathbb{R}_{+} ; E_{0}\right)}+\|w-v\|_{C\left(\mathbb{R}_{+} ; E_{0}\right)} .
$$

Перейдем в обеих частях к нижней грани по $w \in \Phi$ :

$$
\inf _{w \in \Phi}\|\mathrm{T}(t) u-w\|_{C\left(\mathbb{R}_{+} ; E_{0}\right)} \leqslant\|\mathrm{T}(t) u-v\|_{C\left(\mathbb{R}_{+} ; E_{0}\right)}+\inf _{w \in \Phi}\|w-v\|_{C\left(\mathbb{R}_{+} ; E_{0}\right)},
$$

и согласно неравенству $(4.2)$

$$
\inf _{w \in \Phi}\|\mathrm{T}(t) u-w\|_{C\left(\mathbb{R}_{+} ; E_{0}\right)} \leqslant\|\mathrm{T}(t) u-v\|_{C\left(\mathbb{R}_{+} ; E_{0}\right)}+\frac{\varepsilon}{2} .
$$

Переходя в обеих частях полученного неравенства к нижней грани по $v \in \Phi_{\alpha}$, а затем к верхней грани по $u \in B$, получаем:

$$
\sup _{u \in B} \inf _{w \in \Phi}\|\mathrm{T}(t) u-w\|_{C\left(\mathbb{R}_{+} ; E_{0}\right)} \leqslant \sup _{u \in B} \inf _{v \in \Phi_{\alpha}}\|\mathrm{T}(t) u-v\|_{C\left(\mathbb{R}_{+} ; E_{0}\right)}+\frac{\varepsilon}{2},
$$

а с учетом (4.3) имеем

$$
\sup _{u \in B} \inf _{w \in \Phi}\|\mathrm{T}(t) u-w\|_{C\left(\mathbb{R}_{+} ; E_{0}\right)} \leqslant \varepsilon .
$$

Это неравенство выполняется при произвольном $\varepsilon>0$ для всех $t \geqslant h$, где $h>0$ определяется по $\varepsilon$, что и означает притяжение.

Таким образом, $\Phi$ - траекторный полуаттрактор. Покажем, что он содержится в любом полуаттракторе. Пусть $P^{\prime}$ - полуаттрактор. Тогда $P^{\prime} \cap P$ - также полуаттрактор; следовательно, $P^{\prime} \cap P$ является некоторым множеством $\Phi_{\alpha}$, и

$$
\Phi \subset \Phi_{\alpha}=P^{\prime} \cap P \subset P^{\prime}
$$

что и требовалось доказать. 
Несложно проверить, что при любом $t \geqslant 0$ множество $\mathrm{T}(t) \Phi-$ полуаттрактор. По доказанному имеем

$$
\Phi \subset \mathrm{T}(t) \Phi
$$

Последнее включение означает, что $\Phi$ является и квазиаттрактором, т. е. $\Phi-$ траекторный аттрактор. Так как $\Phi$ содержится в каждом полуаттракторе, то $\Phi$ содержится и в каждом аттракторе, т. е. $\Phi$ - минимальный траекторный аттрактор.

Доказательство остальных утверждений теоремы имеет технический характер, и мы его опускаем. Теорема 4.1 доказана.

На практике удобнее доказывать существование поглощающего, а не притягивающего множества.

ОПРеДЕЛЕНИЕ 4.7. Множество $P \subset C\left(\mathbb{R}_{+} ; E_{0}\right) \cap L_{\infty}\left(\mathbb{R}_{+} ; E\right)$ называется поглощающим для $\mathscr{H}^{+}$, если для каждого множества $B \subset \mathscr{H}^{+}$, ограниченного в норме $L_{\infty}\left(\mathbb{R}_{+} ; E\right)$, найдется $t_{B} \geqslant 0$ такое, что

$$
\mathrm{T}(t) B \subset P \quad \text { при } t \geqslant t_{B} .
$$

С точки зрения приложений представляет интерес приводимое ниже следствие теоремы 4.1 [14].

СлЕДСТВИЕ 4.2. Пусть существует компактное в $C\left(\mathbb{R}_{+} ; E_{0}\right)$ и ограниченное в $L_{\infty}\left(\mathbb{R}_{+} ; E\right)$ поглощающее множество $P$ для пространства траекторий $\mathscr{H}^{+}$. Тогда существует минимальный траекторный аттрактор $\mathscr{U}$ пространства траекторий $\mathscr{H}^{+}$, имеют место соотношения

$$
\Pi_{+} \mathscr{K}\left(\mathscr{H}^{+}\right) \subset \mathscr{U}=\Pi_{+} \mathscr{K}(\mathscr{U}) \subset \Pi_{+} \mathscr{K}(P) \subset P
$$

и ядро $\mathscr{K}\left(\mathscr{H}^{+}\right)$относительно компактно в $C\left(\mathbb{R} ; E_{0}\right)$ и ограничено в $L_{\infty}(\mathbb{R} ; E)$.

Доказательство следствия заключается в том, что показывается, что замыкание в $C\left(\mathbb{R}_{+} ; E_{0}\right)$ множества

$$
P_{0}=\bigcup_{B} \bigcup_{t \geqslant h(B)} \mathrm{T}(t) B
$$

является полуаттрактором пространства $\mathscr{H}^{+}$(внешнее объединение берется по всем ограниченным подмножествам $\mathscr{H}^{+}$, а $h(B)$ - такое число, что $\mathrm{T}(t) B \subset P$ при $t \geqslant h(B))$.

Следующее утверждение [14] может облегчить применение теоремы 4.1 и следствия 4.2 .

Лемма 4.2. Пусть $P$ - относительно компактное в $C\left(\mathbb{R}_{+} ; E_{0}\right)$ и ограниченное в $L_{\infty}\left(\mathbb{R}_{+} ; E\right)$ притягивающее (соответственно поглощающее) множество для пространства траекторий $\mathscr{H}^{+}$. Тогда его замыкание $\bar{P}$ в пространстве $C\left(\mathbb{R}_{+} ; E_{0}\right)$ является компактным в $C\left(\mathbb{R}_{+} ; E_{0}\right)$ и ограниченным 
в $L_{\infty}\left(\mathbb{R}_{+} ; E\right)$ притягивающим (соответственно поглощающим) множеством для пространства траекторий $\mathscr{H}^{+}$. Если, кроме того, имеет место включение $\mathrm{T}(t) P \subset P$ при всех $t \geqslant 0$, то $\bar{P}$ - полуаттрактор.

Сформулируем основную теорему о существовании глобального аттрактора неинвариантного пространства траекторий [13], [14].

Теорема 4.2. Пусть существует минимальный траекторный аттрактор $\mathscr{U}$ пространства траекторий $\mathscr{H}^{+}$. Тогда существует глобальный аттрактор А्A пространства $\mathscr{U}$ и справедливы соотношения

$$
\begin{aligned}
\mathscr{A} & =\mathscr{U}(t), & & t \geqslant 0 ; \\
\mathscr{K}\left(\mathscr{H}^{+}\right)(t) \subset \mathscr{A} & =\mathscr{K}(\mathscr{U})(t), & t & \in \mathbb{R} .
\end{aligned}
$$

ДокАзАТЕЛЬство. Из определения аттрактора следует, что $\mathrm{T}(t) \mathscr{U}=\mathscr{U}$, $t \geqslant 0$, откуда ясно, что сечение $\mathscr{U}(t) \subset E$ не зависит от $t$. Обозначим множество $\mathscr{U}(t)$ через $\mathscr{A}$ и покажем, что $\mathscr{A}$ - глобальный аттрактор.

Отображение вычисления $u \mapsto u(t)$ непрерывно в пространствах $C\left(\mathbb{R}_{+} ; E_{0}\right) \rightarrow$ $E_{0}$ при любом $t \geqslant 0$, поэтому множество $\mathscr{A}$ компактно в $E_{0}$ как образ под действием этого отображения компактного множества $\mathscr{U}$. Из ограниченности множества $\mathscr{U}$ в $L_{\infty}\left(\mathbb{R}_{+} ; E\right)$ следует ограниченность его сечения $\mathscr{A}$ в $E$.

Проверим условие притяжения. Пусть множество $B \subset \mathscr{H}^{+}$ограничено в $L_{\infty}\left(\mathbb{R}_{+} ; E\right)$. Это множество притягивается к траекторному аттрактору $\mathscr{U}$, откуда следует, что

$$
\sup _{u \in B} \inf _{v \in \mathscr{U}}\|\mathrm{T}(t) u-v\|_{C\left([0,1] ; E_{0}\right)} \rightarrow 0 \quad(t \rightarrow \infty)
$$

а тогда, принимая во внимание, что $\mathscr{A}=\mathscr{U}(0)$, получаем

$$
\begin{aligned}
\sup _{u \in B} \inf _{y \in \mathscr{A}}\|u(t)-y\|_{E_{0}} & =\sup _{u \in B} \inf _{v \in \mathscr{U}}\|u(t)-v(0)\|_{E_{0}}=\sup _{u \in B} \inf _{v \in \mathscr{U}}\|(\mathrm{T}(t) u-v)(0)\|_{E_{0}} \\
& \leqslant \sup _{u \in B} \inf _{v \in \mathscr{U}}\|\mathrm{T}(t) u-v\|_{C\left([0,1] ; E_{0}\right)} \rightarrow 0 \quad(t \rightarrow \infty)
\end{aligned}
$$

что и означает притяжение семейства решений $B$ к множеству $\mathscr{A}$.

Докажем минимальность множества $\mathscr{A}$. Пусть $\mathscr{A}_{0} \subset E-$ компактное в $E_{0}$ и ограниченное в $E$ множество, обладающее свойством притяжения: для всякого ограниченного в $L_{\infty}\left(\mathbb{R}_{+} ; E\right)$ множества $B \subset \mathscr{H}^{+}$

$$
\sup _{u \in B} \inf _{y \in \mathscr{A}_{0}}\|u(t)-y\|_{E_{0}} \rightarrow 0 \quad(t \rightarrow \infty) .
$$

Покажем, что $\mathscr{A} \subset \mathscr{A}_{0}$. Рассмотрим множество

$$
\mathscr{U}_{0}=\left\{u \in \mathscr{U}: \forall t \geqslant 0 u(t) \in \mathscr{A}_{0}\right\} .
$$

Достаточно убедиться, что $\mathscr{U} \subset \mathscr{U}_{0}$. Сначала покажем, что $\mathscr{U}_{0}-$ полуаттрактор. 
В силу включения $\mathscr{U}_{0} \subset \mathscr{U}$ множество $\mathscr{U}_{0}$ ограничено в $L_{\infty}\left(\mathbb{R}_{+} ; E\right)$ и относительно компактно в $C\left(\mathbb{R}_{+} ; E_{0}\right)$. Покажем, что оно замкнуто в $C\left(\mathbb{R}_{+} ; E_{0}\right)$. Для всякой сходящейся в $C\left(\mathbb{R}_{+} ; E_{0}\right)$ последовательности $\left\{u_{m}\right\} \subset \mathscr{U}_{0}$ ее предел $u_{0}$ принадлежит замкнутому в $C\left(\mathbb{R}_{+} ; E_{0}\right)$ множеству $\mathscr{U}$. Из сходимости в $C\left(\mathbb{R}_{+} ; E_{0}\right)$ следует поточечная сходимость

$$
u_{m}(t) \rightarrow u_{0}(t) \quad \text { в } E_{0} \text { при каждом } t \geqslant 0 .
$$

Множество $\mathscr{A}_{0}$, содержащее последовательность $\left\{u_{m}(t)\right\}$ при любом $t \geqslant 0$, компактно в $E_{0}$ и потому $u_{0}(t) \in \mathscr{A}_{0}$. Таким образом, $u_{0} \in \mathscr{U}_{0}$, и множество $\mathscr{U}_{0}$ замкнуто в $C\left(\mathbb{R}_{+} ; E_{0}\right)$, а следовательно, и компактно в этом пространстве, так как его относительная компактность установлена выше.

Включение $\mathrm{T}(t) \mathscr{U}_{0} \subset \mathscr{U}_{0}, t \geqslant 0$, следует из включения $\mathrm{T}(t) \mathscr{U} \subset \mathscr{U}$.

Покажем, что множество $\mathscr{U}_{0}$ непусто. Пусть $B \subset \mathscr{H}^{+}-$некоторое ограниченное множество, $\left\{u_{m}\right\} \subset B,\left\{h_{m}\right\} \subset \mathbb{R}_{+}, h_{m} \rightarrow \infty$. Расстояние от точки $\mathrm{T}\left(h_{m}\right) u_{m}$ до компакта $\mathscr{U}$ достигается на некоторой функции $w_{m} \in \mathscr{U}$. Последовательность $\left\{w_{m}\right\}$ содержит сходящуюся в $C\left(\mathbb{R}_{+} ; E_{0}\right)$ подпоследовательность $\left\{w_{m_{k}}\right\}$, предел которой обозначим через $w_{0}, w_{0} \in \mathscr{U}$. Так как $\mathscr{U}$ притягивает множество $B$, имеем

$$
\begin{aligned}
\left\|\mathrm{T}\left(h_{m_{k}}\right) u_{m_{k}}-w_{m_{k}}\right\|_{C\left(\mathbb{R}_{+} ; E_{0}\right)} & =\inf _{w \in \mathscr{U}}\left\|\mathrm{T}\left(h_{m_{k}}\right) u_{m_{k}}-w\right\|_{C\left(\mathbb{R}_{+} ; E_{0}\right)} \\
& \leqslant \sup _{u \in B} \inf _{w \in \mathscr{U}}\left\|\mathrm{T}\left(h_{m_{k}}\right) u-w\right\|_{C\left(\mathbb{R}_{+} ; E_{0}\right)} \rightarrow 0 \quad(k \rightarrow \infty),
\end{aligned}
$$

поэтому $\left\{\mathrm{T}\left(h_{m_{k}}\right) u_{m_{k}}\right\}$ сходится к $w_{0}$ вместе с $\left\{w_{m_{k}}\right\}$.

Покажем, что $w_{0} \in \mathscr{U}_{0}$. Предположим, что это не так и существует $t_{0} \geqslant 0$ такое, что $w_{0}\left(t_{0}\right) \notin \mathscr{A}_{0}$. Так как из сходимости в $C\left(\mathbb{R}_{+} ; E_{0}\right)$ следует поточечная сходимость, то

$$
\mathrm{T}\left(h_{m_{k}}\right) u_{m_{k}}\left(t_{0}\right) \rightarrow w_{0}\left(t_{0}\right) \quad \text { в } E_{0} .
$$

Расстояние от $\mathrm{T}\left(h_{m_{k}}\right) u_{m_{k}}\left(t_{0}\right)$ до множества $\mathscr{A}_{0}$, компактного в $E_{0}$, достигается на некотором элементе $y_{m_{k}} \in \mathscr{A}_{0}$, т. е. с учетом условия притяжения (4.6) имеем

$$
\left\|\mathrm{T}\left(h_{m_{k}}\right) u_{m_{k}}\left(t_{0}\right)-y_{m_{k}}\right\|_{E_{0}}=\inf _{y \in \mathscr{A}_{0}}\left\|\mathrm{~T}\left(h_{m_{k}}\right) u_{m_{k}}\left(t_{0}\right)-y\right\|_{E_{0}} \rightarrow 0 \quad(m \rightarrow \infty) .
$$

Отсюда следует, что последовательность $\left\{y_{m_{k}}\right\}$ вместе с $\left\{\mathrm{T}\left(h_{m_{k}}\right) u_{m_{k}}\left(t_{0}\right)\right\}$ сходится к $w_{0}\left(t_{0}\right)$. Это значит, что предел последовательности $\left\{y_{m_{k}}\right\}$, содержащейся в компакте $\mathscr{A}_{0}$, не принадлежит этому компакту. Полученное противоречие означает, что функция $w_{0}$ принадлежит $\mathscr{U}_{0}$. Таким образом, множество $\mathscr{U}_{0}$ непусто.

Покажем, что $\mathscr{U}_{0}$ - притягивающее множество. Предположим, что это не так. Тогда найдутся множество $B \subset \mathscr{H}^{+}$, ограниченное в $L_{\infty}\left(\mathbb{R}_{+} ; E\right)$, и последовательности $\left\{u_{m}\right\} \subset B$ и $\left\{h_{m}\right\} \subset \mathbb{R}_{+}, h_{m} \rightarrow \infty$, такие, что

$$
\operatorname{dist}\left(\mathrm{T}\left(h_{m}\right) u_{m}, \mathscr{U}_{0}\right) \geqslant \varepsilon,
$$


где dist - расстояние в $C\left(\mathbb{R}_{+} ; E_{0}\right)$. Предыдущие рассуждения показывают, что без ограничения общности последовательность $\left\{\mathrm{T}\left(h_{m}\right) u_{m}\right\}$ сходится к $w_{0} \in \mathscr{U}_{0}$. Однако, переходя к пределу в неравенстве $(4.7)$, получаем $\operatorname{dist}\left(w_{0}, \mathscr{U}_{0}\right) \geqslant \varepsilon>0-$ противоречие. Следовательно, $\mathscr{U}_{0}$ - притягивающее множество.

Таким образом, $\mathscr{U}_{0}$ - полуаттрактор. Из доказательства теоремы 4.1 следует включение $\mathscr{U} \subset \mathscr{U}_{0}$. Это означает включение $\mathscr{A} \subset \mathscr{A}_{0}$, и минимальность множества $\mathscr{A}$ доказана. Таким образом, $\mathscr{A}$ - глобальный аттрактор. Вместе с тем доказано представление (4.4).

Соотношения (4.5) без труда выводятся из (4.1). Теорема 4.2 доказана.

Теоремы 4.1 и 4.2 показывают, что в условиях первой из них глобальный аттрактор существует. Оказывается, эти условия можно ослабить и доказать существование глобального аттрактора, привлекая не минимальный траекторный аттрактор, а так называемый однородный квазиаттрактор.

ОПРЕДЕЛЕНиЕ 4.8. Траекторный квазиаттрактор $\mathscr{U}$ пространства траекторий $\mathscr{H}^{+}$называется однородным, если множество $\mathscr{U}(t)$ не зависит от $t \in \mathbb{R}_{+}$.

TеОрема 4.3. Пусть существует компактное в $C\left(\mathbb{R}_{+} ; E_{0}\right)$ и ограниченное в $L_{\infty}\left(\mathbb{R}_{+} ; E\right)$ притягивающее множество $P$ для пространства траекторий $\mathscr{H}^{+}$. Тогда существует такой однородный траекторный квазиаттрактор $\mathscr{U} \subset P$ траекторного пространства $\mathscr{H}^{+}$, что множество $\mathscr{A}=\mathscr{U}(t)$ является глобальным аттрактором для пространства траекторий $\mathscr{H}^{+}$.

Доказательство теоремы можно найти в [14]. Идеи доказательства сходны с идеями доказательств теорем 4.1 и 4.2. Теоретическое значение этой теоремы заключается том, что с ее помощью удобно устанавливать некоторые свойства минимального траекторного аттрактора, содержащегося в пространстве траекторий.

Следующая теорема [14] обобщает теорему 2.2 .

Теорема 4.4. Пусть для пространства траекторий $\mathscr{H}^{+}$существует притягивающее множество $P$, компактное в $C\left(\mathbb{R}_{+} ; E_{0}\right)$ и ограниченное в $L_{\infty}\left(\mathbb{R}_{+} ; E\right)$, и пусть при всех $h \geqslant 0$ выполняется включение $\mathrm{T}(h) P \subset \mathscr{H}^{+}$. Тогда существуют минимальный траекторный аттрактор $\mathscr{U}=\Pi_{+} \mathscr{K}\left(\mathscr{H}^{+}\right)$ и глобальный аттрактор $\mathscr{A}=\mathscr{U}(t)=\mathscr{K}\left(\mathscr{H}^{+}\right)(t), t \geqslant 0$, пространства траекторий $\mathscr{H}^{+}$.

Существенное для приложений в неньютоновской гидродинамике преимущество теоремы 4.4 по сравнению с теоремой М. И. Вишика и В. В. Чепыжова 2.2 состоит в том, что первая не требует инвариантности пространства траекторий.

В определении траекторных аттракторов, полуаттракторов, квазиаттракторов вовсе не требовалось, чтобы эти множества лежали в пространстве траекторий. Таким образом, если пространство траекторий является определенным классом решений какого-то уравнения, то, например, минимальный траекторный аттрактор для этого пространства может не состоять из решений, принадлежащих этому классу. В связи с этим возникает вопрос, из каких же функций состоит траекторный аттрактор. 
Напомним, что на множестве $C\left(\mathbb{R}_{+} ; E_{0}\right) \cap L_{\infty}\left(\mathbb{R}_{+} ; E\right)$ можно задать топологию, объявив множество $P \subset C\left(\mathbb{R}_{+} ; E_{0}\right) \cap L_{\infty}\left(\mathbb{R}_{+} ; E\right)$ замкнутым, если предел всякой последовательности элементов $P$, ограниченной в $L_{\infty}\left(\mathbb{R}_{+} ; E\right)$ и сходящейся в $C\left(\mathbb{R}_{+} ; E_{0}\right)$, принадлежит $P$. Замыкание в этой топологии будем обозначать квадратными скобками. Включение $u \in[P]$ означает, что существует последовательность $\left\{u_{m}\right\} \subset P$, которая ограничена в $L_{\infty}\left(\mathbb{R}_{+} ; E\right)$ и сходится к $u$ в $C\left(\mathbb{R}_{+} ; E_{0}\right)$.

Имеет место следующая теорема [14].

ТеОРема 4.5. Пусть существует минималъный траекторный аттрактор $\mathscr{U}$ пространства траекторий $\mathscr{H}^{+}$. Тогда имеют место включения

$$
\Pi_{+} \mathscr{K}\left(\mathscr{H}^{+}\right) \subset \mathscr{U} \subset \Pi_{+} \mathscr{K}\left(\left[\bigcup_{t \geqslant 0} \mathrm{~T}(t) \mathscr{H}^{+}\right]\right) .
$$

ЗАМЕЧАНИЕ 4.3. Предположим, что пространство траекторий обладает следующими свойствами:

(i) $\mathrm{T}(h) \mathscr{H}^{+} \subset \mathscr{H}^{+}$при всех $h \geqslant 0$ и

(ii) $\left[\mathscr{H}^{+}\right]=\mathscr{H}^{+}$.

Тогда, если существует минимальный траекторный аттрактор $\mathscr{U}$, то по теореме 4.5 имеем $\mathscr{U}=\Pi_{+} \mathscr{K}\left(\mathscr{H}^{+}\right) \subset \mathscr{H}^{+}$, т. е. $\mathscr{U}$ является траекторным аттрактором в смысле определения 2.3. По теореме 4.2 получаем, что $\mathscr{A}=\mathscr{U}(0)-$ глобальный траекторный аттрактор.

В частности, это выполнено для пространства траекторий системы НавьеСтокса, введенного М. И. Вишиком и В. В. Чепыжовым.

Для замкнутого инвариантного пространства траекторий можно сформулировать следующее утверждение [14].

Tеорема 4.6. Пусть существует компактное в $C\left(\mathbb{R}_{+} ; E_{0}\right)$ и ограниченное в $L_{\infty}\left(\mathbb{R}_{+} ; E\right)$ притягивающее множество $P$ для пространства траекторий $\mathscr{H}^{+}$, и пусть выполняются включение $\mathrm{T}(h) \mathscr{H}^{+} \subset \mathscr{H}^{+}$при всех $h \geqslant 0$ и равенство $\left[\mathscr{H}^{+}\right]=\mathscr{H}^{+}$. Тогда однородный квазиаттрактор $\mathscr{U}$, существование которого устанавливается теоремой 4.3, является минимальным траекторным аттрактором и имеют место представления

$$
\mathscr{U}=\Pi_{+} \mathscr{K}\left(\mathscr{H}^{+}\right), \quad \mathscr{A}=\mathscr{K}\left(\mathscr{H}^{+}\right)(t) \quad \forall t \geqslant 0 .
$$

\section{5. Траекторные и глобальные аттракторы ряда задач гидродинамики}

\section{1. Аппроксимационно-топологический подход в задачах гидро-} динамики. Далее мы рассмотрим приложение теории аттракторов неинвариантных пространств траекторий к ряду задач гидродинамики: начально-краевым задачам для модели движения слабоконцентрированных водных растворов полимеров, для регуляризованной модели движения жидких сред с памятью и, более подробно, для системы Джеффриса с полной производной. 
Как правило, в конкретных задачах вопрос о существовании аттракторов тесно связан с теоремами существования решений. Для исследования разрешимости и существования аттракторов рассматриваемых ниже задач гидродинамики используется аппроксимационно-топологический подход, описанный в [19] и развитый в [14].

Суть этого подхода состоит в аппроксимации рассматриваемых уравнений другими уравнениями, обладающими более удобными топологическими свойствами. Такие аппроксимации могут получаться, например, путем сглаживания отдельных членов, путем введения в уравнения членов с производными высоких порядков, путем комбинации этих методов. Аппроксимационные уравнения содержат параметры, при стремлении которых к 0 они переходят в исходные, невозмущенные уравнения.

Аппроксимации строятся таким образом, чтобы для них можно было получать априорные оценки и пользоваться топологическими свойствами операторов (например, компактностью). Разрешимость аппроксимационной задачи обычно устанавливается на основе теории топологический степени: Лере-Шаудера или другой. Может оказаться, что решения аппроксимационной задачи удовлетворяют некоторой оценке диссипативного типа (как правило, содержащей убывающую экспоненту). На такой оценке основывается доказательство существования аттракторов.

После того как разрешимость аппроксимирующей задачи установлена, нужно установить разрешимость невозмущенной задачи с помощью предельного перехода. Здесь важную роль играют априорные оценки, не зависящие от параметров аппроксимации, и теоремы вложения функциональных пространств. Предельный переход дает существование решений исходной задачи. Если некоторые оценки решений аппроксимирующей задачи выдерживают предельный переход, то для исходной задачи получается теорема существования решений, удовлетворяющих определенным оценкам. В частности, таким путем могут быть получены оценки диссипативного типа. На их основе строится пространство траекторий, для которого доказывается существование траекторного и глобального аттракторов.

\section{2. Аттракторы системы Джеффриса с полной производной.}

5.2.1. Система Джеффриса. Движение всякой несжимаемой жидкости постоянной плотности (которую без ограничения общности считаем равной 1) в области $\Omega \in \mathbb{R}^{n}, n=2,3$, описывается системой в форме Коши

$$
\begin{gathered}
\frac{\partial v}{\partial t}+\sum_{i=1}^{n} v_{i} \frac{\partial v}{\partial x_{i}}+\operatorname{grad} p=\operatorname{Div} \sigma+f \\
\operatorname{div} v=0
\end{gathered}
$$

где $v(x, t)$ - вектор скорости частицы жидкости, находящейся в точке $x$ в момент времени $t ; p(x, t)$ - давление жидкости в точке $x$ в момент времени $t$; $f(x, t)$ - вектор плотности внешних сил; $\sigma=\left(\sigma_{i j}(x)\right)$ - девиатор тензора напряжений (симметрическая матрица порядка $n$ ); символ Div означает дивергенцию 
матрицы:

$$
\operatorname{Div} \sigma=\left(\sum_{i=1}^{n} \frac{\partial \sigma_{i 1}}{\partial x_{i}}, \ldots, \sum_{i=1}^{n} \frac{\partial \sigma_{i n}}{\partial x_{i}}\right) .
$$

Далее будем предполагать, что область $\Omega$ ограничена и имеет кусочно липшицеву границу.

В системе (5.1), (5.2) неизвестными являются $v, p, \sigma$, и общее число неизвестных превосходит число уравнений. Поэтому эта система обычно дополняется некоторыми соотношениями между девиатором тензора напряжений $\sigma$ и тензором скорости деформации $\mathscr{E}=\left(\mathscr{E}_{i j}\right)$, который представляет собой симметрическую матрицу с компонентами

$$
\mathscr{E}_{i j}=\mathscr{E}_{i j}(v)=\frac{1}{2}\left(\frac{\partial v_{i}}{\partial x_{j}}+\frac{\partial v_{j}}{\partial x_{i}}\right) \quad(i, j=1, \ldots, n) .
$$

Такие соотношения называются реологическими; различные реологические соотношения описывают различные классы жидкостей. Самое известное реологическое соотношение имеет вид

$$
\sigma=2 \nu \mathscr{E}
$$

при котором система (5.1), (5.2) превращается в уравнения Навье-Стокса.

Реологическое соотношение (5.3) является адекватным для описания движения большого числа встречающихся на практике вязких несжимаемых жидкостей при умеренных скоростях. Однако существуют жидкости с более сложным поведением, для описания которых требуются другие модели. К таковым относятся, например, вязкоупругие среды: они одновременно демонстрируют как вязкие свойства (возникновение сопротивления при перемещении одной части относительно другой), так и упругие (обратимость деформации). Типичным представителем вязкоупругих сред является тело Джефбриса, описывающее такие материалы, как растворы полимеров, битумы, бетон, земная кора (см. [24]).

Реологическое соотношение для тела Джеффриса может быть записано в следующем виде:

$$
\sigma+\lambda_{1} \dot{\sigma}=2 \eta\left(\mathscr{E}+\lambda_{2} \dot{\mathscr{E}}\right)
$$

Точкой в соотношении (5.4) обозначена некоторая производная по времени, характер которой уточним ниже. Параметр $\eta>0$ называется вязкостъю тела Джеффриса. Параметр $\lambda_{1}>0$ называется временем релаксации, а параметр $\lambda_{2}>0$ - временем ретардации (или последействия), причем $\lambda_{2}<\lambda_{1}$. Из соотношения (5.4) можно усмотреть смысл двух последних параметров: именно, если тело Джеффриса подвергнуто постоянной деформации $(\dot{\mathscr{E}}=0)$, то напряжение $\sigma$ экспоненциально убывает и время релаксации $\lambda_{1}$ характеризует скорость этого убывания; с другой стороны, при отсутствии напряжения $(\dot{\sigma}=0)$ скорость деформации $\mathscr{E}$ экспоненциально убывает и скорость убывания характеризуется временем ретардации $\lambda_{2}$. 
Для конкретных жидкостей, обладающих вязкоупругими свойствами модели Джеффриса, параметры $\lambda_{1}, \lambda_{2}$ и $\eta$ могут быть измерены экспериментально.

Как было сказано выше, точка в уравнении (5.4) означает некоторую производную по времени. Эту производную можно понимать в различных смыслах, и от выбора производной зависит получаемая модель. Так, самый простой вариант состоит в том, чтобы рассмотреть частную производную $\partial / \partial t$. Мы же рассмотрим более физичную модель с полной (или субстанииальной) производной

$$
\frac{d}{d t}=\frac{\partial}{\partial t}+\sum_{i=1}^{n} v_{i} \frac{\partial}{\partial x_{i}}
$$

Эта производная соответствует дифференцированию вдоль линий тока жидкости.

Реологическое соотношение Джеффриса с полной производной имеет вид

$$
\sigma+\lambda_{1}\left(\frac{\partial \sigma}{\partial t}+\sum_{i=1}^{n} v_{i} \frac{\partial \sigma}{\partial x_{i}}\right)=2 \eta\left(\mathscr{E}+\lambda_{2}\left(\frac{\partial \mathscr{E}}{\partial t}+\sum_{i=1}^{n} v_{i} \frac{\partial \mathscr{E}}{\partial x_{i}}\right)\right)
$$

Уравнения (5.1), (5.2), (5.5) образуют систему Джефбриса с полной производной. Мы рассматриваем начально-краевую задачу для системы Джеффриса в ограниченной области $\Omega \subset \mathbb{R}^{n}(n=2,3)$. На границе ставится условие прилипания

$$
\left.v\right|_{\partial \Omega}=0
$$

а начальное условие имеет вид

$$
\left.v\right|_{t=0}=a,\left.\quad \sigma\right|_{t=0}=\sigma_{0}
$$

где функции $a$ и $\sigma_{0}$ произвольным образом выбираются из некоторых функциональных пространств.

В ряде вопросов, касающихся системы Джеффриса (в частности, при исследовании существования слабых решений и их аттракторов), удобно ввести новые обозначения. Положим

$$
\mu_{1}=\frac{\eta \lambda_{2}}{\lambda_{1}}, \quad \mu_{2}=\frac{\eta-\mu_{1}}{\lambda_{1}}
$$

(отметим, что $\mu_{1}>0$ и $\mu_{2}>0$ в силу неравенств $\eta>0,0<\lambda_{2}<\lambda_{1}$ ) и произведем замену в системе (5.1), (5.2), (5.5): вместо неизвестной функции $\sigma$ будем рассматривать матричнозначную функцию

$$
\tau=\sigma-2 \mu_{1} \mathscr{E}(v)
$$


После этой подстановки задача (5.1), (5.2), (5.5)-(5.7) переходит в эквивалентную задачу

$$
\begin{gathered}
\frac{\partial v}{\partial t}-\mu_{1} \Delta v+\sum_{i=1}^{n} v_{i} \frac{\partial v}{\partial x_{i}}-\operatorname{Div} \tau=f \\
\frac{\partial \tau}{\partial t}+\frac{\tau}{\lambda_{1}}+\sum_{i=1}^{n} v_{i} \frac{\partial \tau}{\partial x_{i}}=2 \mu_{2} \mathscr{E}(v) \\
\operatorname{div} v=0 \\
\left.v\right|_{\partial \Omega}=0 \\
\left.v\right|_{t=0}=a,\left.\quad \tau\right|_{t=0}=\tau_{0}
\end{gathered}
$$

Задача (5.9)-(5.13) и будет рассматриваться в дальнейшем.

Уравнения (5.9) и (5.11) отличаются от системы Навье-Стокса присутствием в левой части уравнения (5.9) слагаемого $-\operatorname{Div} \tau$.

В настоящем разделе будут рассмотрены траекторный и глобальный аттракторы автономной системы Джеффриса (т. е. в случае, когда плотность внешних сил $f$ не зависит от времени).

Введем некоторые пространства матричных функций.

Пространство симметрических матриц порядка $n$ будем обозначать $M_{S}(n)$. Это конечномерное евклидово пространство, в котором скалярное произведение матриц $A=\left(a_{i j}\right)$ и $B=\left(b_{i j}\right)$ задается формулой

$$
A: B=\operatorname{tr} A B=\sum_{i, j=1}^{n} a_{i j} b_{i j}
$$

Для пространств функций со значениями в $M_{S}(n)$ будем использовать естественные обозначения: например, $L_{2}\left(\Omega ; M_{S}(n)\right)$ - гильбертово пространство функций с суммируемым квадратом, скалярное произведение в котором задается формулой

$$
(\sigma, \tau)=\int_{\Omega} \sigma(x): \tau(x) d x .
$$

Также будем рассматривать соболевские пространства матричных функций, например, $H^{s}\left(\Omega ; M_{S}(n)\right)(s \geqslant 0)$ - при целом $s$ это пространство функций, принадлежащих $L_{2}\left(\Omega ; M_{S}(n)\right)$ вместе со своими обобщенными производными до порядка $s$ включительно. Если нет опасности недоразумения, то будем писать просто $L_{2}$ вместо $L_{2}\left(\Omega ; M_{S}(n)\right)$ и т. д.; особо отметим, что при рассмотрении системы Джеффриса обозначение $C_{0}^{\infty}$ будет использоваться исключительно для множества таких бесконечно гладких функций, заданных на замыкании области $\Omega$ и принимающих значения в $M_{S}(n)$, носитель которых компактен и содержится в $\Omega$.

Обозначим $H_{0}^{s}\left(\Omega ; M_{S}(n)\right)$ замыкание множества $C_{0}^{\infty}$ в соболевском пространстве $H^{s}\left(\Omega ; M_{S}(n)\right)$; пространство, сопряженное к $H_{0}^{s}$, будем обозначать $H^{-s}$. 
Гильбертово пространство $L_{2}$ по теореме Рисса можно отождествить с его сопряженным, и с учетом этого отождествления имеем цепочку плотных вложений

$$
H_{0}^{2} \subset H_{0}^{1} \subset H_{0}^{\alpha} \subset L_{2} \equiv\left(L_{2}\right)^{*} \subset H^{-\alpha} \subset H^{-1} \subset H^{-2} .
$$

Здесь $\alpha \in(0,1)$ - фиксированное число. Самым широким из пространств матричных функций, которое будем использовать, является $H^{-2}$. Производные функций числового аргумента со значениями в $H^{-2}$ будем понимать в смысле распределений со значениями в $H^{-2}$.

Скалярное произведение в пространствах суммируемых с квадратом векторных или матричных функций будет обозначаться круглыми скобками, а действие линейного функционала на вектор - угловыми.

Для пространств функций числового аргумента со значениями в пространствах матричных функций будем использовать стандартные обозначения: например, $L_{\infty}\left(0, T ; L_{2}\right)$.

Отметим некоторые следствия неравенства Пуанкаре (1.8):

$$
\begin{aligned}
& \|u\|_{H} \leqslant K_{0}\|u\|_{V}, \quad u \in V, \\
& \|\sigma\|_{L_{2}} \leqslant K_{0}\|\nabla \sigma\|_{L_{2}}, \quad \sigma \in H_{0}^{1},
\end{aligned}
$$

где

$$
\|\nabla \sigma\|_{L_{2}}^{2}=\sum_{i, j, k}\left\|\frac{\partial \sigma_{i j}}{\partial x_{k}}\right\|_{L_{2}(\Omega)}^{2} .
$$

Пока система Джеффриса рассматривается на конечном отрезке $t \in[0, T]$, будем считать, что плотность внешних сил $f$ принадлежит $L_{2}\left(0, T ; V^{*}\right)$; когда перейдем к исследованию аттракторов, будем считать, что $f \in V^{*}-$ функция, не зависящая от времени, т. е. задача (5.10)-(5.13) автономна.

Пусть $a \in H, \tau_{0} \in L_{2}$. Под слабым решением задачи (5.9)-(5.13) понимается пара $(v, \tau)$ функций классов

$$
\left.\begin{array}{rl}
v & \in L_{2}(0, T ; V) \cap L_{\infty}(0, T ; H) \cap C_{w}(0, T ; H), \\
v^{\prime} & \in L_{1}\left(0, T ; V^{*}\right) ; \\
\tau & \in L_{\infty}\left(0, T ; L_{2}\right) \cap C_{w}\left(0, T ; L_{2}\right), \\
\tau^{\prime} & \in L_{2}\left(0, T ; H^{-2}\right),
\end{array}\right\}
$$

удовлетворяющих тождествам

$$
\begin{array}{rlrl}
\frac{d}{d t}(\tau, \Phi)+\frac{1}{\lambda_{1}}(\tau, \Phi)-\sum_{i=1}^{n}\left(v_{i} \tau, \frac{\partial \Phi}{\partial x_{i}}\right)+2 \mu_{2}(v, \operatorname{Div} \Phi) & =0 & & \left(\Phi \in C_{0}^{\infty}\right), \\
\frac{d}{d t}(v, \varphi)-\sum_{i=1}^{n}\left(v_{i} v, \frac{\partial \varphi}{\partial x_{i}}\right)+\mu_{1}(\nabla v, \nabla \varphi)+(\tau, \nabla \varphi) & =\langle f, \varphi\rangle & (\varphi \in \mathscr{V})
\end{array}
$$


почти всюду на $(0, T)$ при любых $\varphi \in \mathscr{V}$ и $\Phi \in C_{0}^{\infty}$ и начальным условиям

$$
v(0)=a, \quad \tau=\tau_{0}
$$

Тождества (5.19) и (5.20) получаются из уравнений (5.9), (5.10) путем умножения последних на пробные функции и интегрирования по частям.

При изучении аттракторов задачи (5.9)-(5.13) числовые параметры $\lambda_{1}, \mu_{1}, \mu_{2}$, а также функцию $f$ считаем фиксированными. С другой стороны, начальные условия (5.13) произвольным образом выбираются из соответствующих пространств.

Имеются следующие результаты о существования решений (см. [14], [26]).

Теорема 5.1. Пусть данъ функиии $f \in L_{2}\left(0, T ; V^{*}\right), a \in H, \tau_{0} \in L_{2}$. Тогда существует пара функиии $(v, \tau)$ классов (5.17), (5.18), удовлетворяющих при любых $\varphi \in \mathscr{V}, \Phi \in C_{0}^{\infty}$ тождествам (5.19), (5.20) для почти всех $t \in(0, T)$, а такэе удовлетворяющих начальным условиям (5.21) и оценке

$$
\begin{aligned}
& \|v\|_{L_{2}(0, T ; V)}+\|v\|_{L_{\infty}(0, T ; H)}+\left\|v^{\prime}\right\|_{L_{1}\left(0, T ; V^{*}\right)} \\
& \quad+\|\tau\|_{L_{\infty}\left(0, T ; L_{2}\right)}+\left\|\tau^{\prime}\right\|_{L_{2}\left(0, T ; H^{-2}\right)} \leqslant K_{1}\left(\|a\|,\left\|\tau_{0}\right\|,\|f\|_{L_{2}\left(0, T ; V^{*}\right)}\right)
\end{aligned}
$$

с постоянной $K_{1}$, непрерьвно зависящей от $\|a\|,\left\|\tau_{0}\right\|,\|f\|_{L_{2}\left(0, T ; V^{*}\right)}$ и не зависящей от области $\Omega$.

В работах [13], [25] для доказательства разрешимости задачи (5.19)-(5.21) и существования ее аттракторов используется аппроксимационно-топологический подход. Тождества (5.19) и (5.20) аппроксимируются тождествами

$$
\begin{aligned}
& \frac{d}{d t}(\tau, \Phi)+\frac{1}{\lambda_{1}}(\tau, \Phi)-\sum_{i=1}^{n}\left(\frac{v_{i} \tau}{1+\xi\left(|\tau|^{2} /\left(2 \mu_{2}\right)+|v|^{2}\right)}, \frac{\partial \Phi}{\partial x_{i}}\right) \\
& +2 \mu_{2}(v, \operatorname{Div} \Phi)+\frac{\varepsilon}{\lambda_{1}}(\nabla \tau, \nabla \Phi)=0 \quad(\Phi \in V), \\
& \frac{d}{d t}(v, \varphi)-\sum_{i=1}^{n}\left(\frac{v_{i} v}{1+\xi\left(|\tau|^{2} /\left(2 \mu_{2}\right)+|v|^{2}\right)}, \frac{\partial \varphi}{\partial x_{i}}\right)+\mu_{1}(\nabla v, \nabla \varphi) \\
& +(\tau, \nabla \varphi)=\langle f, \varphi\rangle \quad\left(\varphi \in H_{0}^{1}\left(\Omega, M_{S}(n)\right)\right)
\end{aligned}
$$

(здесь $\xi>0,0<\varepsilon \leqslant 1$ - параметры). Как видим, аппроксимация состоит в добавлении члена высшего порядка с малым параметром $\varepsilon$ и в одновременной регуляризации нелинейных конвективных членов. Эта модификация служит тому, чтобы для полученных уравнений можно было устанавливать априорные оценки и доказывать разрешимость топологическими методами, а именно с помощью теории степени Лере-Шаудера. После этого с помощью предельного перехода устанавливается существование решений невозмущенной задачи, удовлетворяющих оценкам, необходимым для построения аттракторов. 
Чтобы сформулировать теорему существования и предельного перехода, введем пространства, в которых ищутся решения аппроксимационной задачи:

$$
\begin{gathered}
W=W(T)=\left\{v \in L_{2}(0, T ; V): v^{\prime} \in L_{2}\left(0, T ; V^{*}\right)\right\}, \\
\|v\|_{W}=\|v\|_{L_{2}(0, T ; V)}+\left\|v^{\prime}\right\|_{L_{2}\left(0, T ; V^{*}\right)} ; \\
W_{M}=W_{M}(T)=\left\{\tau \in L_{2}\left(0, T ; H_{0}^{1}\left(\Omega, M_{S}(n)\right)\right):\right. \\
\left.\tau^{\prime} \in L_{2}\left(0, T ; H^{-1}\left(\Omega, M_{S}(n)\right)\right)\right\}, \\
\|\tau\|_{W_{M}}=\|\tau\|_{L_{2}\left(0, T ; H_{0}^{1}\right)}+\left\|\tau^{\prime}\right\|_{L_{2}\left(0, T ; H^{-1}\right)} .
\end{gathered}
$$

ЗАмечАниЕ 5.1. Пространство $W$ вкладывается в $C([0, T] ; H)$ в том смысле, что каждый класс эквивалентности из $W$ содержит единственную непрерывную функцию со значениями в $H$; аналогичным образом, пространство $W_{M}$ вкладывается в $C\left([0, T] ; L_{2}\right)$. Это следует из леммы 1.1 .

Имеет место следующая теорема [25].

Teорема 5.2. Пусть $\xi>0,0<\varepsilon \leqslant 1, a \in H, \tau_{0} \in L_{2}, f \in L_{2}\left(0, T ; V^{*}\right)$. Тогда задача (5.23), (5.24), (5.21) имеет решение $v \in W, \tau \in W_{M}$. Если последовательности $\left\{\varepsilon_{\mu}\right\} \quad u\left\{\xi_{\nu}\right\}$ сходятся $\kappa 0 u\left(v_{\mu \nu}, \tau_{\mu \nu}\right)-$ решения задачи (5.23), (5.24), (5.21), соответствующие $\varepsilon=\varepsilon_{\mu} u \xi=\xi_{\nu}$, то для некоторьх подпоследовательностей существуют повторные предель

$$
\begin{aligned}
v & =\lim _{k \rightarrow \infty} \lim _{l \rightarrow \infty} v_{\mu_{k} \nu_{l}}, \\
\tau & =\lim _{k \rightarrow \infty} \lim _{l \rightarrow \infty} \tau_{\mu_{k} \nu_{l}}
\end{aligned}
$$

(в (5.25) предель можно понимать в смысле слабой сходимости в $L_{2}(0, T ; V)$ u *-слабой сходимости в $L_{\infty}(0, T ; H)$, а в (5.26) - в смысле *-слабой сходимости в $\left.L_{\infty}\left(0, T ; L_{2}\right)\right)$; пара предельных функиий $(v, \tau)$ является решением задачи (5.19)-(5.21) в классах (5.17), (5.18).

5.2.2. Оценки решений. Следующая лемма дает тождество, которому удовлетворяют нормы функций, удовлетворяющих аппроксимационным тождествам $(5.23),(5.24)$. Этот результат нужен нам для доказательства существования аттракторов.

Лемма 5.1. Пусть $f \in L_{2}\left(0, T ; V^{*}\right)$. Тогда всякая пара функиий $(v, \tau) \in$ $W \times W_{M}$, удовлетворяющая тождествам (5.23), (5.24) на отрезке $[0, T]$, удовлетворяет почти всюду на $(0, T)$ равенству

$$
\begin{gathered}
\frac{1}{2} \frac{d}{d t}\|v(t)\|_{H}^{2}+\frac{1}{4 \mu_{2}} \frac{d}{d t}\|\tau(t)\|_{L_{2}}^{2}+\mu_{1}\|v(t)\|_{V}^{2}+\frac{1}{2 \lambda_{1} \mu_{2}}\|\tau(t)\|_{L_{2}}^{2} \\
+\frac{\varepsilon}{2 \lambda_{1} \mu_{2}}\|\nabla \tau(t)\|_{L_{2}}^{2}=\langle f(t), v(t)\rangle .
\end{gathered}
$$

ДокАЗАТЕЛьСтво. Подставим в тождества (5.23), (5.24)

$$
\varphi=v(t), \quad \Phi=\tau(t)
$$


С учетом леммы 1.1 получаем

$$
\begin{gathered}
\frac{1}{2} \frac{d}{d t}\|\tau(t)\|_{L_{2}}^{2}+\frac{1}{\lambda_{1}}\|\tau(t)\|_{L_{2}}^{2}-\sum_{i=1}^{n}\left(\frac{v_{i}(t) \tau(t)}{1+\xi\left(|\tau(t)|^{2} /\left(2 \mu_{2}\right)+|v(t)|^{2}\right)}, \frac{\partial \tau(t)}{\partial x_{i}}\right) \\
+2 \mu_{2}(v(t), \operatorname{Div} \tau(t))+\frac{\varepsilon}{\lambda_{1}}\|\nabla \tau(t)\|_{L_{2}}=0, \\
\frac{1}{2} \frac{d}{d t}\|v(t)\|_{H}^{2}-\sum_{i=1}^{n}\left(\frac{v_{i}(t) v(t)}{1+\xi\left(|\tau(t)|^{2} /\left(2 \mu_{2}\right)+|v(t)|^{2}\right)}, \frac{\partial v(t)}{\partial x_{i}}\right)+\mu_{1}\|v(t)\|_{V}^{2} \\
+(\tau(t), \nabla v(t))=\langle f, v(t)\rangle .
\end{gathered}
$$

Умножим первое равенство на $1 /\left(2 \mu_{2}\right)$ и сложим его со вторым; с учетом того, что для любых $u \in\left(H_{0}^{1}(\Omega)\right)^{n}$ и $\sigma \in W_{2}^{1}\left(\Omega, M_{S}(n)\right)$ при $\mu_{2}, \xi>0$ выполняются тождества

$$
\begin{gathered}
(\sigma, \nabla u)+(u, \operatorname{Div} \sigma)=0 \\
\sum_{i=1}^{n}\left(\frac{u_{i} u}{1+\xi\left(|\sigma|^{2} /\left(2 \mu_{2}\right)+|u|^{2}\right)}, \frac{\partial u}{\partial x_{i}}\right) \\
+\frac{1}{2 \mu_{2}} \sum_{i=1}^{n}\left(\frac{u_{i} \sigma}{1+\xi\left(|\sigma|^{2} /\left(2 \mu_{2}\right)+|u|^{2}\right)}, \frac{\partial \sigma}{\partial x_{i}}\right)=0
\end{gathered}
$$

получаем (5.27). Лемма 5.1 доказана.

Тождество (5.27) является аналогом тождества (1.17) и неравенства (3.5) для двумерной и трехмерной системы Навье-Стокса соответственно. Однако тождество (5.27) выполняется только для аппроксимационной системы и не выдерживает предельного перехода даже в обобщенном виде. Тем не менее из дифференциального тождества (5.27) можно вывести оценку диссипативного типа, которая сохраняется при предельном переходе от решений аппроксимационной задачи к решениям исходной задачи. Такая оценка выводится в нижеследующей теореме. Ее характерной чертой является то, что начальные условия входят в нее с множителем $e^{-2 \gamma t}$, что соответствует их "забыванию" с течением времени.

Tеорема 5.3. Пусть $f \in V^{*}, T>0$. Тогда для любъх $a \in H, \tau_{0} \in L_{2}$ на отрезке $[0, T]$ существует решение $(v, \tau)$ задачи (5.19)-(5.21), удовлетворяющее оценке

$$
\begin{gathered}
\frac{1}{4}\|v\|_{L_{\infty}(t, t+1 ; H)}^{2}+\frac{1}{8 \mu_{2}}\|\tau\|_{L_{\infty}\left(t, t+1 ; L_{2}\right)}^{2}+\frac{\mu_{1}}{2}\|v\|_{L_{2}(t, t+1 ; V)}^{2} \\
\leqslant \frac{\gamma+1}{2 \mu_{1} \gamma}\|f\|_{V^{*}}^{2}+\left(\|a\|_{H}^{2}+\frac{1}{2 \mu_{2}}\|\tau\|_{L_{2}}^{2}\right) e^{-2 \gamma t}
\end{gathered}
$$

при $t \in[0, T-1]$ (здесь $\gamma=\min \left\{\frac{1}{\lambda_{1}}, \frac{\mu_{1}}{2 K_{0}^{2}}\right\}$ - число, завислщее от области и числовых параметров задачи, $K_{0}$ - постоянная из неравенств (5.15), (5.16)). 
ДоказАтельство. I этап. Покажем, что оценка (5.29) выполняется для любого решения $(v, \tau)$ аппроксимационной задачи $(5.23),(5.24),(5.21)$ с произвольными $\xi>0$ и $\varepsilon \in(0,1]$.

Сначала получим оценку двух первых слагаемых в левой части неравенства (5.29). Положим

$$
\bar{v}(t)=e^{\gamma t} v(t), \quad \bar{\tau}(t)=e^{\gamma t} \tau(t)
$$

Подставив новые функции в тождество (5.27), получаем

$$
\begin{aligned}
\frac{1}{2} \frac{d}{d t}\left(e^{-2 \gamma t}\|\bar{v}\|_{H}^{2}\right)+\frac{1}{4 \mu_{2}} \frac{d}{d t}\left(e^{-2 \gamma t}\|\bar{\tau}\|_{L_{2}}^{2}\right)+\mu_{1}\left(e^{-2 \gamma t}\|\bar{v}\|_{V}^{2}\right) \\
\quad+\frac{1}{2 \lambda_{1} \mu_{2}}\left(e^{-2 \gamma t}\|\bar{\tau}\|_{L_{2}}^{2}\right)+\frac{\varepsilon}{2 \lambda_{1} \mu_{2}}\left(e^{-2 \gamma t}\|\nabla \bar{\tau}\|_{L_{2}}^{2}\right)=\left\langle f, e^{-\gamma t} \bar{v}\right\rangle
\end{aligned}
$$

Раскрыв производную произведения и умножив обе части на $e^{2 \gamma t}$, получим

$$
\begin{aligned}
\frac{1}{2} \frac{d}{d t}\|\bar{v}\|_{H}^{2}-\gamma\|\bar{v}\|_{H}^{2} & +\frac{1}{4 \mu_{2}} \frac{d}{d t}\|\bar{\tau}\|_{L_{2}}^{2}-\frac{\gamma}{2 \mu_{2}}\|\bar{\tau}\|_{L_{2}}^{2}+\mu_{1}\|\bar{v}\|_{V}^{2} \\
& +\frac{1}{2 \lambda_{1} \mu_{2}}\|\bar{\tau}\|_{L_{2}}^{2}+\frac{\varepsilon}{2 \lambda_{1} \mu_{2}}\|\nabla \bar{\tau}\|_{L_{2}}^{2}=e^{\gamma t}\langle f, \bar{v}\rangle
\end{aligned}
$$

С учетом определения числа $\gamma$ имеем

$$
\begin{aligned}
&-\gamma\|\bar{v}\|_{H}^{2}+\mu_{1}\|\bar{v}\|_{V}^{2} \geqslant-\frac{\mu_{1}}{2 K_{0}^{2}} \cdot K_{0}^{2}\|\bar{v}\|_{V}^{2}+\mu_{1}\|\bar{v}\|_{V}^{2} \geqslant \frac{\mu_{1}}{2}\|\bar{v}\|_{V}^{2}, \\
&-\frac{\gamma}{2 \mu_{2}}\|\bar{\tau}\|_{L_{2}}^{2}+\frac{1}{2 \lambda_{1} \mu_{2}}\|\bar{\tau}\|_{L_{2}}^{2} \geqslant-\frac{1}{2 \lambda_{1} \mu_{2}}\|\bar{\tau}\|_{L_{2}}^{2}+\frac{1}{2 \lambda_{1} \mu_{2}}\|\bar{\tau}\|_{L_{2}}^{2}=0 .
\end{aligned}
$$

С помощью этих двух оценок из тождества (5.30) выводим неравенство

$$
\frac{1}{2} \frac{d}{d t}\|\bar{v}\|_{H}^{2}+\frac{1}{4 \mu_{2}} \frac{d}{d t}\|\bar{\tau}\|_{L_{2}}^{2}+\frac{\mu_{1}}{2}\|\bar{v}\|_{V}^{2} \leqslant e^{\gamma t}\|f\|_{V^{*}}\|\bar{v}\|_{V} .
$$

Применяя к произведению в правой части неравенство Коши, получаем

$$
\frac{1}{2} \frac{d}{d t}\|\bar{v}\|_{H}^{2}+\frac{1}{4 \mu_{2}} \frac{d}{d t}\|\bar{\tau}\|_{L_{2}}^{2}+\frac{\mu_{1}}{2}\|\bar{v}\|_{V}^{2} \leqslant \frac{e^{2 \gamma t}}{2 \mu_{1}}\|f\|_{V^{*}}^{2}+\frac{\mu_{1}}{2}\|\bar{v}\|_{V}^{2},
$$

откуда следует, что

$$
\frac{1}{2} \frac{d}{d t}\|\bar{v}\|_{H}^{2}+\frac{1}{4 \mu_{2}} \frac{d}{d t}\|\bar{\tau}\|_{L_{2}}^{2} \leqslant \frac{e^{2 \gamma t}}{2 \mu_{1}}\|f\|_{V^{*}}^{2}
$$

Проинтегрировав последнее неравенство от 0 до $t$, находим, что

$$
\frac{1}{2}\|\bar{v}\|_{H}^{2}+\frac{1}{4 \mu_{2}}\|\bar{\tau}\|_{L_{2}}^{2} \leqslant \frac{1}{2}\|a\|_{H}^{2}+\frac{1}{4 \mu_{2}}\left\|\tau_{0}\right\|_{L_{2}}^{2}+\frac{1}{4 \gamma \mu_{1}}\|f\|_{V^{*}}^{2}\left(e^{2 \gamma t}-1\right) .
$$


Возвратившись к исходным функциям, получаем

$$
\frac{1}{2}\left\|e^{\gamma t} v\right\|_{H}^{2}+\frac{1}{4 \mu_{2}}\left\|e^{\gamma t} \tau\right\|_{L_{2}}^{2} \leqslant \frac{1}{2}\|a\|_{H}^{2}+\frac{1}{4 \mu_{2}}\left\|\tau_{0}\right\|_{L_{2}}^{2}+\frac{1}{4 \gamma \mu_{1}}\|f\|_{V^{*}}^{2}\left(e^{2 \gamma t}-1\right) .
$$

Умножим обе части на $2 e^{-2 \gamma t}$ и заменим $t$ на $s$, имеем:

$$
\|v(s)\|_{H}^{2}+\frac{1}{2 \mu_{2}}\|\tau(s)\|_{L_{2}}^{2} \leqslant 2 e^{-2 \gamma s}\left(\|a\|_{H}^{2}+\frac{1}{2 \mu_{2}}\left\|\tau_{0}\right\|_{L_{2}}^{2}\right)+\frac{1}{2 \gamma \mu_{1}}\|f\|_{V^{*}}^{2}\left(1-e^{-2 \gamma s}\right) .
$$

Функции $\|v\|_{H}$ и $\|\tau\|_{L_{2}}$ непрерывны на $[0, T]$ (см. замечание 5.1), поэтому последнее неравенство выполняется всюду на $[0, T]$ и можно перейти к максимуму по отрезку $[t, t+1] \subset[0, T]$. Получаем оценку

$$
\begin{aligned}
& \max _{s \in[t, t+1]}\left(\|v(s)\|_{H}^{2}+\frac{1}{2 \mu_{2}}\|\tau(s)\|_{L_{2}}^{2}\right) \\
& \quad \leqslant e^{-2 \gamma t}\left(\|a\|_{H}^{2}+\frac{1}{2 \mu_{2}}\left\|\tau_{0}\right\|_{L_{2}}^{2}\right)+\frac{1}{2 \gamma \mu_{1}}\|f\|_{V^{*}}^{2}
\end{aligned}
$$

Возвратимся к тождеству (5.27), чтобы получить оценку третьего слагаемого в левой части неравенства (5.29). Оценив правую часть тождества (5.27) с помощью неравенства Коши:

$$
|\langle f, v(t)\rangle| \leqslant\|f\|_{V^{*}}\|v(t)\|_{V} \leqslant \frac{1}{2 \mu_{1}}\|f\|_{V^{*}}^{2}+\frac{\mu_{1}}{2}\|v(t)\|_{V}^{2},
$$

приходим к неравенству

$$
\frac{1}{2} \frac{d}{d t}\|v(t)\|_{H}^{2}+\frac{1}{4 \mu_{2}} \frac{d}{d t}\|\tau(t)\|_{L_{2}}^{2}+\frac{\mu_{1}}{2}\|v(t)\|_{V}^{2}+\frac{\varepsilon}{2 \lambda_{1} \mu_{2}}\|\nabla \tau(t)\|_{L_{2}}^{2} \leqslant \frac{1}{2 \mu_{1}}\|f\|_{V^{*}}^{2} .
$$

Заменяя $t$ на $s$ и интегрируя по отрезку $[t, t+1] \subset[0, T]$, получаем

$$
\begin{aligned}
& \frac{1}{2}\|v(t+1)\|_{H}^{2}-\frac{1}{2}\|v(t)\|_{H}^{2}+\frac{1}{4 \mu_{2}}\|\tau(t+1)\|_{L_{2}}^{2}-\frac{1}{4 \mu_{2}}\|\tau(t)\|_{L_{2}}^{2} \\
& \quad+\frac{\mu_{1}}{2} \int_{t}^{t+1}\|v(s)\|_{V}^{2} d s+\frac{\varepsilon}{2 \lambda_{1} \mu_{2}} \int_{t}^{t+1}\|\nabla \tau(s)\|_{L_{2}}^{2} d s \leqslant \frac{1}{2 \mu_{1}}\|f\|_{V^{*}}^{2} .
\end{aligned}
$$

В силу непрерывности функций $v$ и $\tau$ на $[0, T]$ со значениями в $H$ и $L_{2}$ соответственно последнее неравенство выполняется всюду на $[0, T-1]$. Складывая его с неравенством (5.31), находим, что

$$
\begin{gathered}
\frac{1}{2}\|v(t+1)\|_{H}^{2}+\frac{1}{4 \mu_{2}}\|\tau(t+1)\|_{L_{2}}^{2} \\
\quad+\max _{s \in[t, t+1]}\left(\frac{1}{2}\|v(s)\|_{H}^{2}+\frac{1}{4 \mu_{2}}\|\tau(s)\|_{L_{2}}^{2}\right) \\
\quad+\frac{\mu_{1}}{2} \int_{t}^{t+1}\|v(s)\|_{V}^{2} d s+\frac{\varepsilon}{2 \lambda_{1} \mu_{2}} \int_{t}^{t+1}\|\nabla \tau(s)\|_{L_{2}}^{2} d s \\
\leqslant e^{-2 \gamma t}\left(\|a\|_{H}^{2}+\frac{1}{2 \mu_{2}}\left\|\tau_{0}\right\|_{L_{2}}^{2}\right)+\frac{1+\gamma}{2 \gamma \mu_{1}}\|f\|_{V^{*}}^{2} .
\end{gathered}
$$


Из последнего неравенства легко получается оценка $(5.29)$ решения $(v, \tau)$ аппроксимационной задачи: достаточно отбросить неотрицательные слагаемые в левой части и воспользоваться очевидным неравенством

$$
\max (A+B) \geqslant \frac{1}{2}(\max A+\max B),
$$

справедливым для неотрицательных величин $A$ и $B$.

II эman. Доказательство существования решений задачи (5.19)-(5.21), удовлетворяющих оценке (5.29), осуществляется с помощью предельного перехода. Рассмотрим сходящиеся к 0 числовые последовательности $\left\{\varepsilon_{\mu}\right\}$ и $\left\{\xi_{\nu}\right\}$ такие, что $0<\varepsilon_{\mu} \leqslant 1, \xi_{\nu}>0$, и пусть $\left(v_{\mu \nu}, \tau_{\mu \nu}\right)$ - решения на отрезке $[0, T]$ задачи $(5.23),(5.24),(5.21)$, соответствующие $\varepsilon=\varepsilon_{\mu}$ и $\xi=\xi_{\nu}$. Согласно теореме 5.2 без ограничения общности можно считать, что существуют повторные пределы

$$
v=\lim _{\mu \rightarrow \infty} \lim _{\nu \rightarrow \infty} v_{\mu \nu}, \quad \tau=\lim _{\mu \rightarrow \infty} \lim _{\nu \rightarrow \infty} \tau_{\mu \nu}
$$

(повторный предел функций $v_{\mu \nu}$ понимается в слабой топологии пространства $L_{2}(0, T ; V)$ и *-слабой топологии пространства $L_{\infty}(0, T ; H)$; повторный предел функций $\tau_{\mu \nu}$ понимается в смысле $*$-слабой сходимости в $\left.L_{\infty}\left(0, T ; L_{2}\right)\right)$; предельные функции $(v, \tau)$ являются решением задачи (5.19)-(5.21). По ранее доказанному для решений аппроксимационной задачи при $t \in[0, T-1]$ выполняется неравенство

$$
\begin{gathered}
\frac{1}{4}\left\|v_{\mu \nu}\right\|_{L_{\infty}(t, t+1 ; H)}^{2}+\frac{1}{8 \mu_{2}}\left\|\tau_{\mu \nu}\right\|_{L_{\infty}\left(t, t+1 ; L_{2}\right)}^{2}+\frac{\mu_{1}}{2}\left\|v_{\mu \nu}\right\|_{L_{2}(t, t+1 ; V)}^{2} \\
\leqslant \frac{\gamma+1}{2 \mu_{1} \gamma}\|f\|_{V^{*}}^{2}+\left(\|a\|_{H}^{2}+\frac{1}{2 \mu_{2}}\left\|\tau_{0}\right\|_{L_{2}}^{2}\right) e^{-2 \gamma t}
\end{gathered}
$$

с правой частью, не зависящей от $\mu$ и $\nu$. Устремляя последовательно $\nu$ и $\mu \mathrm{k} \infty$ и принимая во внимание полунепрерывность сверху нормы в слабой и $*$-слабой топологиях, получаем неравенство (5.29). Теорема 5.3 доказана.

Для использования теорем вложения требуется получить оценки производных $v^{\prime}$ и $\tau^{\prime}$ функций, удовлетворяющих тождествам (5.19), (5.20). Это можно сделать, выразив в этих тождествах члены, содержащие производные, и оценив остальные члены. Этот путь приводит к следующему утверждению.

Лемма 5.2. Пусть пара функций $(v, \tau)$ классов (5.17), (5.18) удовлетворяет тождествам (5.19), (5.20) для любых $\varphi \in \mathscr{V}, \Phi \in C_{0}^{\infty}$. Тогда $v^{\prime} \in$ $L_{4 / 3}\left(0, T ; V^{*}\right)$ и при $0 \leqslant t \leqslant T-1$ имеет место оценка

$$
\begin{aligned}
& \left\|v^{\prime}\right\|_{L_{4 / 3}\left(t, t+1 ; V^{*}\right)}+\left\|\tau^{\prime}\right\|_{L_{2}\left(t, t+1 ; H^{-2}\right)} \\
& \leqslant C \\
& \quad\left(\|v\|_{L_{\infty}(t, t+1 ; H)}^{1 / 2}\|v\|_{L_{2}(t, t+1 ; V)}^{3 / 2}+\|v\|_{L_{2}(t, t+1 ; V)}\right. \\
& \quad+\|\tau\|_{L_{\infty}\left(t, t+1 ; L_{2}\right)}+\|v\|_{L_{2}(t, t+1 ; V)}\|\tau\|_{L_{\infty}\left(t, t+1 ; L_{2}\right)} \\
& \left.\quad+\|v\|_{L_{\infty}(t, t+1 ; H)}+\|f\|_{V^{*}}\right)
\end{aligned}
$$

c постоянной $C$, не зависящей от $v, \tau$. 
5.2.3. Траекторный и глобальный аттракторы. Пусть $\alpha \in(0,1]$. Обозначим через $V_{\alpha}$ замкнутое подпространство соболевского пространства $\left(W_{2}^{\alpha}\right)^{n}$, в котором плотно множество $\mathscr{V}$ гладких финитных бездивергентных векторных полей на области $\Omega$, и пусть $V_{\alpha}^{*}$ - сопряженное к $V_{\alpha}$ пространство. С учетом отождествления $H \equiv H^{*}$ имеем вложение $H \subset V_{\alpha}^{*}$.

Для введения пространства траекторий системы Джеффриса будем использовать банаховы пространства

$$
E=H \times L_{2}\left(\Omega, M_{s}(n)\right) \quad \text { и } \quad E_{0}=V_{\alpha}^{*} \times H^{-\alpha}\left(\Omega, M_{s}(n)\right), \quad \text { где } \alpha \in(0,1] .
$$

В качестве пространства траекторий рассмотрим множество $\mathscr{H}^{+}$, состоящее из пар функций $(v, \tau)$, которые:

(i) принадлежат классу

$$
v \in L_{2}^{\text {loc }}\left(\mathbb{R}_{+} ; V\right) \cap L_{\infty}\left(\mathbb{R}_{+} ; H\right), \quad \tau \in L_{\infty}\left(\mathbb{R}_{+} ; L_{2}\right) ;
$$

(ii) удовлетворяют интегральным тождествам (5.19), (5.20) для всех $\varphi \in \mathscr{V}$, $\Phi \in C_{0}^{\infty}$ при почти всех $t \in \mathbb{R}_{+}$;

(iii) удовлетворяют энергетической оценке

$$
\begin{aligned}
& \frac{1}{4}\|v\|_{L_{\infty}(t, t+1 ; H)}^{2}+\frac{1}{8 \mu_{2}}\|\tau\|_{L_{\infty}\left(t, t+1 ; L_{2}\right)}^{2}+\frac{\mu_{1}}{2}\|v\|_{L_{2}(t, t+1 ; V)}^{2} \\
& \leqslant \frac{\gamma+1}{2 \mu_{1} \gamma}\|f\|_{V^{*}}^{2}+\left(\|v\|_{L_{\infty}\left(\mathbb{R}_{+} ; H\right)}^{2}+\frac{1}{2 \mu_{2}}\|\tau\|_{L_{\infty}\left(\mathbb{R}_{+} ; L_{2}\right)}^{2}\right) e^{-2 \gamma t},
\end{aligned}
$$

где $\gamma$ - постоянная из теоремы 5.3.

ЗАмЕчАниЕ 5.2. Если пара $(v, \tau)$ принадлежит определенному выше пространству траекторий, то функции $v$ и $\tau$ имеют производные

$$
v^{\prime} \in L_{4 / 3}^{\text {loc }}\left(\mathbb{R}_{+} ; V^{*}\right) \quad \text { и } \quad \tau^{\prime} \in L_{2}^{\text {loc }}\left(\mathbb{R}_{+} ; H^{-2}\right),
$$

что следует из леммы 5.2 .

Чтобы данное выше определение пространства траекторий было корректным, нужно убедиться, что пространство непусто, и проверить включение

$$
\mathscr{H}^{+} \subset C\left(\mathbb{R}_{+} ; E_{0}\right) \cap L_{\infty}\left(\mathbb{R}_{+} ; E\right) .
$$

Сначала займемся вторым вопросом. Включение $\mathscr{H}^{+} \subset L_{\infty}\left(\mathbb{R}_{+} ; E\right)$ непосредственно следует из пункта (i) определения пространства траекторий. Докажем непрерывность траекторий. Пусть $(v, \tau)$ - некоторая траектория. Выберем произвольное число $T>0$. Согласно замечанию 5.2 имеем $v \in L_{\infty}(0, T ; H)$, $v^{\prime} \in L_{4 / 3}\left(0, T ; V^{*}\right)$, поэтому из леммы 3.1 для тройки пространств $H \subset V_{\alpha}^{*} \subset V^{*}$ следует, что $v$ принадлежит пространству $C\left([0, T] ; V_{\alpha}^{*}\right)$. Это верно при любом $T$, так что $v \in C\left(\mathbb{R}_{+} ; V_{\alpha}^{*}\right)$, что и требовалось доказать. Непрерывность функции $\tau$ доказывается аналогично: пусть $T>0$; функция $\tau$ принадлежит 
пространству $L_{\infty}\left(0, T ; L_{2}\right)$, а ее производная - пространству $L_{2}\left(0, T ; H^{-2}\right)$; применяя лемму $3.1 \mathrm{k}$ тройке пространств $L_{2} \subset H^{-\alpha} \subset H^{-2}$, делаем вывод, что $\tau$ непрерывна на $[0, T]$ со значениями в $H^{-2}$. В силу произвольности $T$ имеем непрерывность на всей полуоси.

Следующее утверждение показывает не только, что множество $\mathscr{H}^{+}$непусто, но и что оно "богато" траекториями.

Tеорема 5.4. Пусть $f \in V^{*}$. Тогда для каждъьх $a \in H, \tau_{0} \in L_{2}$ существует траектория $\left(v_{*}, \tau_{*}\right) \in \mathscr{H}^{+}$, удовлетворяющая начальным условиям

$$
v_{*}(0)=a, \quad \tau_{*}(0)=\tau_{0} .
$$

Схема ДоказАтельствА теоремы 5.4 следующая. Рассмотрим возрастающую последовательность положительных чисел $T_{m} \rightarrow \infty$. По теореме 5.3 на каждом отрезке $\left[0, T_{m}\right]$ существует решение $\left(v_{m}, \tau_{m}\right)$ в классе $(5.17),(5.18)$ системы (5.19), (5.20), удовлетворяющее начальным условиям

$$
v_{m}(0)=a, \quad \tau_{m}(0)=\tau_{0},
$$

для которого при $t \in\left[0, T_{m}-1\right]$ выполняется неравенство (5.29). Это значит, что при всех $\varphi \in \mathscr{V}, \Phi \in C_{0}^{\infty}$ почти всюду на $\left(0, T_{m}\right)$ выполняются тождества

$$
\begin{gathered}
\frac{d}{d t}\left(\tau_{m}, \Phi\right)+\frac{1}{\lambda_{1}}\left(\tau_{m}, \Phi\right)-\sum_{i=1}^{n}\left(\left(v_{m}\right)_{i} \tau_{m}, \frac{\partial \Phi}{\partial x_{i}}\right)+2 \mu_{2}\left(v_{m}, \operatorname{Div} \Phi\right)=0, \\
\frac{d}{d t}\left(v_{m}, \varphi\right)-\sum_{i=1}^{n}\left(\left(v_{m}\right)_{i} v_{m}, \frac{\partial \varphi}{\partial x_{i}}\right)+\mu_{1}\left(\nabla v_{m}, \nabla \varphi\right)+\left(\tau_{m}, \nabla \varphi\right)=\langle f, \varphi\rangle
\end{gathered}
$$

и при всех $t \in\left[0, T_{m}-1\right]$ выполняется неравенство

$$
\begin{gathered}
\frac{1}{4}\left\|v_{m}\right\|_{L_{\infty}(t, t+1 ; H)}^{2}+\frac{1}{8 \mu_{2}}\left\|\tau_{m}\right\|_{L_{\infty}\left(t, t+1 ; L_{2}\right)}^{2}+\frac{\mu_{1}}{2}\left\|v_{m}\right\|_{L_{2}(t, t+1 ; V)}^{2} \\
\leqslant \frac{\gamma+1}{2 \mu_{1} \gamma}\|f\|_{V^{*}}^{2}+\left(\|a\|_{H}^{2}+\frac{1}{2 \mu_{2}}\left\|\tau_{0}\right\|_{L_{2}}^{2}\right) e^{-2 \gamma t}
\end{gathered}
$$

Из последнего неравенства и леммы 5.2 следует, что решение $\left(v_{m}, \tau_{m}\right)$ удовлетворяет также оценке

$$
\begin{gathered}
\left\|v_{m}\right\|_{L_{\infty}(t, t+1 ; H)}+\left\|\tau_{m}\right\|_{L_{\infty}\left(t, t+1 ; L_{2}\right)}+\left\|v_{m}\right\|_{L_{2}(t, t+1 ; V)} \\
+\left\|v_{m}^{\prime}\right\|_{L_{4 / 3}\left(t, t+1 ; V^{*}\right)}+\left\|\tau_{m}^{\prime}\right\|_{L_{2}\left(t, t+1 ; H^{-2}\right)} \\
\leqslant R_{0}\left(1+\left(\|a\|_{H}^{2}+\left\|\tau_{0}\right\|_{L_{2}}^{2}\right) e^{-2 \gamma t}\right)
\end{gathered}
$$

при $t \in\left[0, T_{m}-1\right]$ с некоторой постоянной $R_{0}$, зависящей только от $\mu_{1}, \mu_{2}, \gamma$ и $\|f\|_{V^{*}}$.

Продолжим с сохранением непрерывности функции $v_{m}$ и $\tau_{m}$ на $\mathbb{R}_{+}$соответственно до функций $\widetilde{v}_{m}, \widetilde{\tau}_{m}$ по следующим формулам:

$$
\widetilde{v}_{m}=\left\{\begin{array}{ll}
v_{m}(t), & 0 \leqslant t \leqslant T_{m}, \\
v_{m}\left(T_{m}\right), & t \geqslant T_{m} ;
\end{array} \quad \widetilde{\tau}_{m}= \begin{cases}\tau_{m}(t), & 0 \leqslant t \leqslant T_{m} \\
\tau_{m}\left(T_{m}\right), & t \geqslant T_{m}\end{cases}\right.
$$


Из неравенства (5.37) следует, что последовательности $\left\{\widetilde{v}_{m}\right\}$ и $\left\{\widetilde{\tau}_{m}\right\}$ ограничены соответственно в $L_{\infty}\left(\mathbb{R}_{+} ; H\right)$ и $L_{\infty}\left(\mathbb{R}_{+} ; L_{2}\right)$. Поэтому без ограничения общности можно считать, что они сходятся $*$-слабо в $L_{\infty}\left(\mathbb{R}_{+} ; H\right)$ и $L_{\infty}\left(\mathbb{R}_{+} ; L_{2}\right)$ соответственно к некоторым функциям $v_{*} \in L_{\infty}\left(\mathbb{R}_{+} ; H\right)$ и $\tau_{*} \in L_{\infty}\left(\mathbb{R}_{+} ; L_{2}\right)$. Также из неравенства (5.37) следует сходимость и в других топологиях: именно, $\left\{v_{m}\right\}$ сходится к $v_{*}$ слабо в $L_{2}(0, T ; V)$ и сильно в $L_{2}(0, T ; H)$ для любого $T>0$. Пользуясь этими сходимостями, можно показать, что пара $\left(v_{*}, \tau_{*}\right)$ удовлетворяет тождествам $(5.19),(5.20)$ для всех $\varphi \in \mathscr{V}, \Phi \in C_{0}^{\infty}$ при почти всех $t \in \mathbb{R}_{+}$, а также начальным условиям (5.35).

Чтобы показать, что $\left(v_{*}, \tau_{*}\right)$ - искомая траектория, остается проверить неравенство (5.34). Фиксируем $t>0$. Неравенство (5.36) выполняется для всех достаточно больших $m$, и, пользуясь полунепрерывностью сверху нормы в слабой топологии, имеем

$$
\begin{aligned}
& \frac{1}{4}\left\|v_{*}\right\|_{L_{\infty}(t, t+1 ; H)}^{2}+\frac{1}{8 \mu_{2}}\left\|\tau_{*}\right\|_{L_{\infty}\left(t, t+1 ; L_{2}\right)}^{2}+\frac{\mu_{1}}{2}\left\|v_{*}\right\|_{L_{2}(t, t+1 ; V)}^{2} \\
& \leqslant \frac{1}{4} \underset{m \rightarrow \infty}{\varliminf_{m \rightarrow \infty}}\left\|v_{m}\right\|_{L_{\infty}(t, t+1 ; H)}^{2}+\frac{1}{8 \mu_{2}} \varliminf_{m \rightarrow \infty}\left\|\tau_{m}\right\|_{L_{\infty}\left(t, t+1 ; L_{2}\right)}^{2} \\
& +\frac{\mu_{1}}{2} \varliminf_{m \rightarrow \infty}\left\|v_{m}\right\|_{L_{2}(t, t+1 ; V)}^{2} \\
& \leqslant \underset{m \rightarrow \infty}{\lim _{m \rightarrow \infty}}\left(\frac{1}{4}\left\|v_{m}\right\|_{L_{\infty}(t, t+1 ; H)}^{2}+\frac{1}{8 \mu_{2}}\left\|\tau_{m}\right\|_{L_{\infty}\left(t, t+1 ; L_{2}\right)}^{2}+\frac{\mu_{1}}{2}\left\|v_{m}\right\|_{L_{2}(t, t+1 ; V)}^{2}\right) \\
& \leqslant \frac{\gamma+1}{2 \mu_{1} \gamma}\|f\|_{V^{*}}^{2}+\left(\|a\|_{H}^{2}+\frac{1}{2 \mu_{2}}\left\|\tau_{0}\right\|_{L_{2}}^{2}\right) e^{-2 \gamma t} \text {. }
\end{aligned}
$$

Из этой оценки и следует неравенство (5.34), так как включения $v \in C_{w}\left(\mathbb{R}_{+}, H\right)$, $\tau \in C_{w}\left(\mathbb{R}_{+}, L_{2}\right)$ влекут за собой соотношения

$$
\begin{aligned}
\|a\|_{H} & =\|v(0)\|_{H} \leqslant\|v\|_{L_{\infty}\left(\mathbb{R}_{+} ; H\right)}, \\
\left\|\tau_{0}\right\|_{L_{2}} & =\|\tau(0)\|_{L_{2}} \leqslant\|\tau\|_{L_{\infty}\left(\mathbb{R}_{+} ; L_{2}\right)}
\end{aligned}
$$

Из предыдущих рассуждений получаем следующее утверждение.

СледСтвиЕ 5.1. Для любой траектории $(v, \tau) \in \mathscr{H}^{+}$выполняется неравенство

$$
\begin{gathered}
\|v\|_{L_{\infty}(t, t+1 ; H)}+\|\tau\|_{L_{\infty}\left(t, t+1 ; L_{2}\right)}+\|v\|_{L_{2}(t, t+1 ; V)} \\
+\left\|v^{\prime}\right\|_{L_{4 / 3}\left(t, t+1 ; V^{*}\right)}+\left\|\tau^{\prime}\right\|_{L_{2}\left(t, t+1 ; H^{-2}\right)} \\
\leqslant R_{0}\left(1+\left(\|v\|_{L_{\infty}\left(\mathbb{R}_{+} ; H\right)}^{2}+\|\tau\|_{L_{\infty}\left(\mathbb{R}_{+} ; L_{2}\right)}^{2}\right) e^{-2 \gamma t}\right)
\end{gathered}
$$

при $t \in \mathbb{R}_{+}$, әде $R_{0}$ - постоянная, зависящая только от $\mu_{1}, \mu_{2}, \gamma u\|f\|_{V^{*}}$.

Основными результатами настоящего раздела являются следующие теоремы о существовании минимального траекторного и глобального аттракторов. 
ТеОрема 5.5 [13]. Пусть $f \in V^{*}$. Тогда существует минимальный траекторный аттрактор $\mathscr{U}$ пространства траекторий $\mathscr{H}^{+}$и выполнены соотношения

$$
\Pi_{+} \mathscr{K}\left(\mathscr{H}^{+}\right) \subset \Pi_{+} \mathscr{K}(\mathscr{U})=\mathscr{U} .
$$

ДокАзАтЕльство. Достаточно доказать существование траекторного полуаттрактора и применить теорему 4.1 .

Рассмотрим множество $P \subset C\left(\mathbb{R}_{+} ; E_{0}\right) \cap L_{\infty}\left(\mathbb{R}_{+} ; E\right)$, состоящее из таких пар $(v, \tau)$, которые при всех $t \geqslant 0$ удовлетворяют неравенству

$$
\begin{aligned}
& \|v\|_{L_{\infty}(t, t+1 ; H)}+\|\tau\|_{L_{\infty}\left(t, t+1 ; L_{2}\right)}+\|v\|_{L_{2}(t, t+1 ; V)} \\
& \quad+\left\|v^{\prime}\right\|_{L_{4 / 3}\left(t, t+1 ; V^{*}\right)}+\left\|\tau^{\prime}\right\|_{L_{2}\left(t, t+1 ; H^{-2}\right)} \leqslant 2 R_{0},
\end{aligned}
$$

где $R_{0}$ - постоянная из неравенства (5.38).

Очевидно, что множество $P$ ограничено в $L_{\infty}\left(\mathbb{R}_{+} ; E\right)$.

Покажем, что множество $P$ относительно компактно в $C\left(\mathbb{R}_{+} ; E_{0}\right)$. Для этого достаточно установить, что при любом $T>0$ множество $\Pi_{T} P$, состоящее из ограничений функций из $P$ на отрезок $[0, T]$, относительно компактно в $C\left([0, T] ; E_{0}\right)$. Однако это следует из того, что сами траектории $(v, \tau) \in P$ ограничены в $L_{\infty}([0, T] ; E)$, а производные $v^{\prime}, \tau^{\prime}$ ограничены в $L_{4 / 3}\left(t, t+1 ; V^{*}\right)$ и $L_{2}\left(t, t+1 ; H^{-2}\right)$ соответственно (лемма 3.1$)$.

Покажем, что множество $P$ замкнуто в $C\left(\mathbb{R} ; E_{0}\right)$ и, следовательно, компактно в этом пространстве. Пусть последовательность $\left(v_{m}, \tau_{m}\right) \subset P$ сходится к $(v, \tau)$ в метрике пространства $C\left(\mathbb{R}_{+} ; E_{0}\right)$. Покажем, что $(v, \tau) \in P$.

Из определения множества $P$ следует выполнение неравенства

$$
\begin{aligned}
& \left\|v_{m}\right\|_{L_{\infty}(t, t+1 ; H)}+\left\|\tau_{m}\right\|_{L_{\infty}\left(t, t+1 ; L_{2}\right)}+\left\|v_{m}\right\|_{L_{2}(t, t+1 ; V)} \\
& \quad+\left\|v_{m}^{\prime}\right\|_{L_{4 / 3}\left(t, t+1 ; V^{*}\right)}+\left\|\tau_{m}^{\prime}\right\|_{L_{2}\left(t, t+1 ; H^{-2}\right)} \leqslant 2 R_{0} \quad(m=1,2, \ldots) .
\end{aligned}
$$

Покажем, что пара $(v, \tau)$ принадлежит классу $C\left(\mathbb{R}_{+} ; E_{0}\right) \cap L_{\infty}\left(\mathbb{R}_{+} ; E\right)$. По построению этой пары имеем $(v, \tau) \in C\left(\mathbb{R}_{+} ; E_{0}\right)$. Далее, из неравенства $(5.41)$ следует, что для любого отрезка $[t, t+1]$ нормы

$$
\underset{s \in(t, t+1)}{\operatorname{vraimax}}\left\|v_{m}(s)\right\|_{H}=\left\|v_{m}\right\|_{L_{\infty}(t, t+1 ; H)}
$$

и

$$
\underset{s \in(t, t+1)}{\operatorname{vrai} \max }\left\|\tau_{m}(s)\right\|_{L_{2}}=\left\|\tau_{m}\right\|_{L_{\infty}\left(t, t+1 ; L_{2}\right)}
$$

ограничены числом $2 R_{0}$, не зависящим от $m$ и $t$. Следовательно, нормы

$$
\underset{s>0}{\operatorname{vraimax}}\left\|v_{m}(s)\right\|_{H}=\left\|v_{m}\right\|_{L_{\infty}\left(\mathbb{R}_{+} ; H\right)}
$$

И

$$
\underset{s>0}{\operatorname{vrai} \max }\left\|\tau_{m}(s)\right\|_{L_{2}}=\left\|\tau_{m}\right\|_{L_{\infty}\left(\mathbb{R}_{+} ; L_{2}\right)}
$$


ограничены тем же самым числом, и последовательности $\left\{v_{m}\right\}$ и $\left\{\tau_{m}\right\}$ сходятся $*$-слабо в $L_{\infty}\left(\mathbb{R}_{+} ; H\right)$ и $L_{\infty}\left(\mathbb{R}_{+} ; L_{2}\right)$ соответственно к своим предельным функциям $v$ и $\tau$. Это значит, что предельные функции принадлежат пространствам $L_{\infty}\left(\mathbb{R}_{+} ; H\right)$ и $L_{\infty}\left(\mathbb{R}_{+} ; L_{2}\right)$, т.е. $(v, \tau) \in L_{\infty}\left(\mathbb{R}_{+} ; E\right)$, что и требовалось доказать.

Рассмотрим произвольный отрезок $[t, t+1], t \geqslant 0$. Так как $\left(v_{m}, \tau_{m}\right) \rightarrow(v, \tau)$ в $C\left(\mathbb{R}_{+} ; E_{0}\right)$, то

$$
v_{m} \rightarrow v \quad \text { в } C\left([t, t+1] ; V^{-\alpha}\right) \quad \text { и } \quad \tau_{m} \rightarrow \tau \quad \text { в } C\left([t, t+1] ; H^{-\alpha}\right) .
$$

Нормы, стоящие в левой части неравенства (5.41), ограничены, поэтому $v_{m}$ сходится к $v$ слабо в $L_{2}(t, t+1 ; V)$ и $*$-слабо в $L_{\infty}(t, t+1 ; H)$, а $\tau_{m}$ сходится к $\tau$ *-слабо в $L_{\infty}\left(t, t+1 ; L_{2}\right)$; далее, существуют подпоследовательности $v_{m_{k}}^{\prime}$ и $\tau_{m_{k}}^{\prime}$ такие, что

$$
v_{m_{k}}^{\prime} \rightarrow u \quad \text { слабо в } L_{4 / 3}\left(t, t+1 ; V^{*}\right)
$$

и

$$
\tau_{m_{k}}^{\prime} \rightarrow \sigma \quad \text { слабо в } L_{2}\left(t, t+1 ; H^{-2}\right) .
$$

Однако в смысле распределений $\mathscr{D}^{\prime}\left(t, t+1 ; V^{*}\right)$ имеем $v_{m_{k}}^{\prime} \rightarrow v^{\prime}$, а в смысле распределений $\mathscr{D}^{\prime}\left(t, t+1 ; H^{-2}\right)$ имеем $\tau_{m_{k}}^{\prime} \rightarrow \tau^{\prime}$; таким образом,

$$
v_{m_{k}}^{\prime} \rightarrow v^{\prime} \quad \text { слабо в } L_{4 / 3}\left(t, t+1 ; V^{*}\right)
$$

и

$$
\tau_{m_{k}}^{\prime} \rightarrow \tau^{\prime} \quad \text { слабо в } L_{2}\left(t, t+1 ; H^{-2}\right) .
$$

Пользуясь верхней полунепрерывностью нормы в слабой топологии, для предельных функций получаем:

$$
\begin{aligned}
& \|v\|_{L_{\infty}(t, t+1 ; H)}+\|\tau\|_{L_{\infty}\left(t, t+1 ; L_{2}\right)}+\|v\|_{L_{2}(t, t+1 ; V)} \\
& +\left\|v^{\prime}\right\|_{L_{4 / 3}\left(t, t+1 ; V^{*}\right)}+\left\|\tau^{\prime}\right\|_{L_{2}\left(t, t+1 ; H^{-2}\right)} \\
& \leqslant \underline{\lim _{k \rightarrow \infty}}\left\|v_{m_{k}}\right\|_{L_{\infty}(t, t+1 ; H)}+\underline{\lim _{k \rightarrow \infty}}\left\|\tau_{m_{k}}\right\|_{L_{\infty}\left(t, t+1 ; L_{2}\right)}+\underline{\lim _{k \rightarrow \infty}}\left\|v_{m_{k}}\right\|_{L_{2}(t, t+1 ; V)} \\
& +\varliminf_{k \rightarrow \infty}\left\|v_{m_{k}}^{\prime}\right\|_{L_{4 / 3}\left(t, t+1 ; V^{*}\right)}+\varliminf_{k \rightarrow \infty}\left\|\tau_{m_{k}}^{\prime}\right\|_{L_{2}\left(t, t+1 ; H^{-2}\right)} \\
& \leqslant \underline{\lim _{k \rightarrow \infty}}\left(\left\|v_{m_{k}}\right\|_{L_{\infty}(t, t+1 ; H)}+\left\|\tau_{m_{k}}\right\|_{L_{\infty}\left(t, t+1 ; L_{2}\right)}+\left\|v_{m_{k}}\right\|_{L_{2}(t, t+1 ; V)}\right. \\
& \left.+\left\|v_{m_{k}}^{\prime}\right\|_{L_{4 / 3}\left(t, t+1 ; V^{*}\right)}+\left\|\tau_{m_{k}}^{\prime}\right\|_{L_{2}\left(t, t+1 ; H^{-2}\right)}\right) \leqslant 2 R_{0},
\end{aligned}
$$

т. е. пара $(v, \tau)$ удовлетворяет неравенству (5.40) и, таким образом, принадлежит множеству $P$. Замкнутость и компактность множества $P$ доказаны.

Покажем, что множество $P$ является поглощающим. Пусть $B \subset \mathscr{H}^{+}$ множество, ограниченное в $L_{\infty}\left(\mathbb{R}_{+} ; E\right)$; пусть для $(v, \tau) \in B$ выполнены неравенства

$$
\|v\|_{L_{\infty}\left(\mathbb{R}_{+} ; H\right)} \leqslant R, \quad\|\tau\|_{L_{\infty}\left(\mathbb{R}_{+} ; L_{2}\right)} \leqslant R
$$


Пусть число $h_{0}$ таково, что

$$
2 R^{2} e^{-2 \gamma h_{0}} \leqslant 1
$$

Тогда для $(v, \tau) \in B$ при $h \geqslant h_{0}$ в соответствии с неравенством (5.38) имеем

$$
\begin{gathered}
\|\mathrm{T}(h) v\|_{L_{\infty}(t, t+1 ; H)}+\|\mathrm{T}(h) \tau\|_{L_{\infty}\left(t, t+1 ; L_{2}\right)}+\|\mathrm{T}(h) v\|_{L_{2}(t, t+1 ; V)} \\
+\left\|\mathrm{T}(h) v^{\prime}\right\|_{L_{4 / 3}\left(t, t+1 ; V^{*}\right)}+\left\|\mathrm{T}(h) \tau^{\prime}\right\|_{L_{2}\left(t, t+1 ; H^{-2}\right)} \\
=\|v\|_{L_{\infty}(h+t, h+t+1 ; H)}+\|\tau\|_{L_{\infty}\left(h+t, h+t+1 ; L_{2}\right)}+\|v\|_{L_{2}(h+t, h+t+1 ; V)} \\
\quad+\left\|v^{\prime}\right\|_{L_{4 / 3}\left(h+t, h+t+1 ; V^{*}\right)}+\left\|\tau^{\prime}\right\|_{L_{2}\left(h+t, h+t+1 ; H^{-2}\right)} \\
\leqslant R_{0}\left(1+2 R^{2} e^{-2 \gamma(t+h)}\right) \leqslant 2 R_{0} .
\end{gathered}
$$

Итак, для пары $(\mathrm{T}(h) v, \mathrm{~T}(h) \tau)$ неравенство (5.40) выполняется, и, следовательно, $\mathrm{T}(h) B \subset P$ при $h \geqslant h_{0}$. Это значит, что множество $P$ действительно является поглощающим.

Таким образом, доказано, что $P$ - ограниченное в $L_{\infty}\left(\mathbb{R}_{+} ; E\right)$, компактное в $C\left(\mathbb{R}_{+} ; E_{0}\right)$ поглощающее (следовательно, и притягивающее) множество для пространства траекторий $\mathscr{H}^{+}$. Кроме того, очевидно включение $\mathrm{T}(h) P \subset P$, $h \geqslant 0$. Это значит, что множество $P$ является искомым траекторным полуаттрактором. Как было сказано выше, существования траекторного полуаттрактора достаточно для существования минимального траекторного аттрактора.

Соотношения (5.39) справедливы по теореме 4.1. Теорема 5.5 доказана.

Tеорема 5.6 [13]. Пусть $f \in V^{*}$. Тогда существует глобальный аттрактор $\mathscr{A}$ пространства траекторий $\mathscr{H}^{+}$и выполнены соотношения

$$
\mathscr{K}\left(\mathscr{H}^{+}\right)(t) \subset \mathscr{A}=\mathscr{U}(t)=\mathscr{K}(\mathscr{U})(t) \quad(t \geqslant 0) .
$$

ДокАзАтельство. Утверждение следует из существования минимального траекторного аттрактора, доказанного в предыдущей теореме, и общей теоремы 4.2 .

5.3. Аттракторы модели движения слабоконцентрированных водных растворов полимеров. Математической моделью движения слабоконцентрированных водных растворов полимеров называется система

$$
\begin{gathered}
\frac{\partial v}{\partial t}-\nu \Delta v+\sum_{i=1}^{n} v_{i} \frac{\partial v}{\partial x_{i}}-\varkappa \frac{\partial \Delta v}{\partial t}-2 \varkappa \operatorname{Div}\left(\sum_{i=1}^{n} v_{i} \frac{\partial \mathscr{E}(v)}{\partial x_{i}}\right)+\operatorname{grad} p=f \\
\operatorname{div} v=0
\end{gathered}
$$

где $v(x, t)$ - вектор скорости частицы жидкости, находящейся в точке $x$ в момент времени $t ; p(x, t)$ - давление жидкости в точке $x$ в момент времени $t$; $f(x, t)$ - вектор плотности внешних сил; $\mathscr{E}=\left(\mathscr{E}_{i j}\right)$ - тензор скоростей деформации, $\nu>0$ - кинематический коэффициент вязкости, $\varkappa>0$ - время ретардации.

Уравнение (5.42) соответствует реологическому соотношению

$$
\sigma=2 \nu\left(\mathscr{E}+\varkappa \nu^{-1} \frac{d \mathscr{E}}{d t}\right),
$$

которое было предложено в [26] на основании исследований [27]. 
Уравнения (5.42), (5.43) будем рассматривать в ограниченной области $\Omega \subset$ $\mathbb{R}^{n}(n=2,3)$ с гладкой границей. Система снабжается граничным условием прилипания и начальным условием:

$$
\left.v\right|_{\partial \Omega}=0,\left.\quad v\right|_{t=0}=a,
$$

где $a$ - функция из некоторого функционального пространства.

Разрешимость задачи (5.42)-(5.44) рассматривалась в работах [28], [29]. Следуя [15], рассмотрим для автономного случая (когда плотность внешних сил $f$ не зависит от времени) вопрос о существовании минимального траекторного и глобального аттракторов этой задачи.

Пусть $\left\{V^{\alpha}\right\}$ - шкала пространств, порожденная оператором Стокса (см. п. 3.1). Норма в $V^{\alpha}$ обозначается $\|\cdot\|_{\alpha}$.

Для определения слабого решения на отрезке введем следующие пространства:

$$
\begin{aligned}
& W_{1}[0, T]=\left\{v: v \in L_{\infty}\left(0, T ; V^{1}\right), v^{\prime} \in L_{\infty}\left(0, T ; V^{-1}\right)\right\} \\
& W_{2}[0, T]=\left\{v: v \in C\left([0, T] ; V^{3}\right), v^{\prime} \in L_{\infty}\left(0, T ; V^{3}\right)\right\}
\end{aligned}
$$

Для определения слабого решения на полуоси $\mathbb{R}_{+}$будет рассматриваться пространство $W_{1}^{\text {loc }}\left(\mathbb{R}_{+}\right)$, состоящее из функций $v$, определенных почти всюду на $\mathbb{R}_{+}$ и принимающих значения в $V^{1}$ таких, что ограничение $v$ на любой отрезок $[0, T]$ принадлежит $W_{1}[0, T]$, и пространство $W_{2}^{\text {loc }}\left(\mathbb{R}_{+}\right)$, состоящее из функций $v$ класса $C\left(\mathbb{R}_{+} ; V^{3}\right)$ таких, что ограничение $v$ на любой отрезок $[0, T]$ принадлежит $W_{2}[0, T]$.

Пусть плотность внешних сил $f$ принадлежит пространству $\left(L_{2}(\Omega)\right)^{n}$.

ОПРЕДЕЛЕНИЕ 5.1. Слабым решением задачи (5.42)-(5.44) на отрезке $[0, T]$ будем называть функцию $v \in W_{1}[0, T]$ такую, что тождество

$$
\begin{aligned}
& \frac{d}{d t} \int_{\Omega} v(t) \varphi d x+\varkappa \frac{d}{d t} \int_{\Omega} \nabla v(t): \nabla \varphi d x+\nu \int_{\Omega} \nabla v(t): \nabla \varphi d x \\
& \quad-\sum_{i, j=1}^{n} \int_{\Omega} v_{i}(t) v_{j}(t) \frac{\partial \varphi_{j}}{\partial x_{i}} d x-\varkappa \sum_{i, j, k=1}^{n} \int_{\Omega} v_{k}(t) \frac{\partial v_{i}}{\partial x_{j}}(t) \frac{\partial^{2} \varphi_{j}}{\partial x_{i} \partial x_{k}} d x \\
& \quad-\varkappa \sum_{i, j, k=1}^{n} \int_{\Omega} v_{k}(t) \frac{\partial v_{j}}{\partial x_{i}}(t) \frac{\partial^{2} \varphi_{j}}{\partial x_{i} \partial x_{k}} d x=\int_{\Omega} f \varphi d x
\end{aligned}
$$

выполнено почти всюду на $(0, T)$ для любой функции $\varphi \in V^{3}$. Слабъм решением системы (5.42)-(5.44) на полуоси $\mathbb{R}_{+}$будем называть функцию $v \in W_{1}^{\text {loc }}\left(\mathbb{R}_{+}\right)$ такую, что при каждом $T>0$ ограничение $v$ на отрезок $[0, T]$ является решением этой системы на этом отрезке.

Тождество (5.45) получается из системы (5.42), (5.43) стандартным образом: в предположении, что имеется классическое решение (и правая часть $f$ достаточно гладка), уравнение (5.42) умножается на пробную функцию $\varphi \in V^{3}$, 
после чего в некоторых членах производится интегрирование по частям; вследствие соленоидальности функции $\varphi$ член $\operatorname{grad} p$ исключается из уравнения.

Введем постоянную

$$
\gamma=\frac{\nu}{K_{0}^{2}+\varkappa}
$$

ОПРЕДЕЛЕНИЕ 5.2. В качестве пространства траекторий $\mathscr{H}^{+}$системы (5.42), (5.43) будем рассматривать множество решений этой системы, определенных на $\mathbb{R}_{+}$, существенно ограниченных на $\mathbb{R}_{+}$со значениями в $V^{1}$ и удовлетворяющих оценке

$$
\|v(t)\|_{1}+\left\|v^{\prime}(t)\right\|_{-1} \leqslant R_{0}\left(1+\|v\|_{L_{\infty}\left(\mathbb{R}_{+} ; V^{1}\right)}^{2} e^{-\gamma t}\right)
$$

при почти всех $t>0$ (здесь $R_{0}$ - некоторая постоянная, не зависящая от $v$ и зависящая только от параметров задачи).

Показывается [15], что каждый элемент пространства $V^{1}$ служит началом по крайней мере одной траектории.

Сформулируем основные результаты о существовании аттракторов (см. [15]). Зафиксируем $\alpha \in(0,1]$.

Теорема 5.7. Пусть $f \in\left(L_{2}(\Omega)\right)^{n}$. Тогда существует минималъный траекторный аттрактор $\mathscr{U}$ пространства траекторий $\mathscr{H}^{+}$. Аттрактор ограничен в $L_{\infty}\left(\mathbb{R}_{+} ; V^{1}\right)$, компактен в $C\left(\mathbb{R}_{+} ; V^{1-\alpha}\right) ;$ он притягивает в топологии пространства $C\left(\mathbb{R}_{+} ; V^{1-\alpha}\right)$ семейства траекторий, ограниченные в пространcmве $L_{\infty}\left(\mathbb{R}_{+} ; V^{1}\right)$.

Теорема 5.8. Пусть $f \in\left(L_{2}(\Omega)\right)^{n}$. Тогда существует глобальный аттрактор $\mathscr{A}$ пространства траекторий $\mathscr{H}^{+}$. Аттрактор ограничен в $V^{1}$, компактен в $V^{1-\alpha}$; он притягивает в топологии пространства $V^{1-\alpha}$ семейства траекторий, ограниченнве в $L_{\infty}\left(\mathbb{R}_{+} ; V^{1}\right)$.

Для доказательства этих теорем рассматривается множество $P$, состоящее из таких функций $v \in C\left(\mathbb{R}_{+} ; V^{1-\alpha}\right) \cap L_{\infty}\left(\mathbb{R}_{+} ; V^{1}\right)$, что $v^{\prime} \in L_{\infty}\left(\mathbb{R}_{+} ; V^{-1}\right)$ и при почти всех $t \geqslant 0$ выполнено неравенство

$$
\|v(t)\|_{1}+\left\|v^{\prime}(t)\right\|_{-1} \leqslant 2 R_{0}
$$

(здесь $R_{0}-$ постоянная из неравенства $(5.46)$ ).

Легко видеть, что $P$ - поглощающее множество. Действительно, пусть множество $B \subset \mathscr{H}^{+}$ограничено в $L_{\infty}\left(\mathbb{R}_{+} ; V^{1}\right)$. Из неравенства (5.46) следует, что существует такое $t_{B} \geqslant 0$, что при $t \geqslant t_{B}$ неравенство (5.47) выполняется для всякой траектории $v \in B$. В свою очередь, отсюда следует, что

$$
\mathrm{T}(h) B \subset P \quad \text { при } t \geqslant t_{B} .
$$

Неравенство (5.47) влечет за собой ограниченность множества $P$ в пространстве $L_{\infty}\left(\mathbb{R}_{+} ; V^{1}\right)$. Положительная инвариантность множества $P$ непосредственно следует из его определения. По лемме 4.2 получаем, что замыкание $\bar{P}$ множества $P$ в топологии пространства $C\left(\mathbb{R}_{+} ; V^{1-\alpha}\right)$ является полуаттрактором 
пространства траекторий $\mathscr{H}^{+}$. Согласно теоремам 4.1 и 4.2, из существования полуаттрактора следует существование минимального траекторного и глобального аттракторов пространства траекторий.

5.4. Аттракторы регуляризованной системы с памятью. Рассмотрим начально-краевую задачу в ограниченной области $\Omega \subset \mathbb{R}^{n}$ с границей $\Gamma$ $(n=2,3)$, соответствующую регуляризованной модели движения жидких сред c памятью:

$$
\begin{aligned}
& \frac{\partial v}{\partial t}+\sum_{i=1}^{n} v_{i} \frac{\partial v}{\partial x_{i}}-\mu_{1} \operatorname{Div} \int_{0}^{t} e^{-(t-s) / \lambda} \mathscr{E}(v)\left(s, Z_{\delta}(s ; t, x)\right) d s-\mu_{0} \operatorname{Div} \mathscr{E}(v) \\
& \quad=-\operatorname{grad} p+\varphi, \quad(t, x) \in(0, T) \times \Omega \\
& \operatorname{div} v=0, \quad(t, x) \in(0, T) \times \Omega ;\left.\quad v\right|_{(0, T) \times \Gamma}=0 \\
& v(0, x)=v^{0}(x), \quad x \in \Omega ; \quad \int_{\Omega} p d x=0
\end{aligned}
$$

где $v=\left(v_{1}, \ldots, v_{n}\right)$ - вектор-функция скорости движения частиц среды, $p-$ давление среды, $\varphi$ - плотность внешних сил, $\mathscr{E}=\left(\mathscr{E}_{i j}\right)$ - тензор скоростей деформации.

Опишем $Z_{\delta}(s ; t, x)$.

Будем использовать те же пространства $H$ и $V$, что и ранее, но на пространстве $V$ в настоящем пункте будет рассматриваться норма

$$
\|u\|_{\mathscr{E}}=\|\mathscr{E}(u)\|_{L_{2}}
$$

(эта норма эквивалентна норме $\|\nabla u\|_{L_{2}}$ ).

Рассмотрим траекторию, определяемую уравнением

$$
z(\tau ; t, x)=x+\int_{t}^{\tau} S_{\delta} v(s, z(s ; t, x)) d s, \quad \tau \in[0, T], \quad(t, x) \in(0, T) \times \Omega .
$$

В уравнении (5.51) используется оператор регуляризации

$$
S_{\delta}: H \rightarrow C^{1}(\Omega)^{n} \cap V \quad \text { для } \delta>0 .
$$

Он обладает следующими свойствами: $S_{\delta}(v) \rightarrow v$ в $H$ при $\delta \rightarrow 0$, и порождаемое им отображение $S_{\delta}: L_{2}(0, T ; H) \rightarrow L_{2}\left(0, T ; C^{1}(\bar{\Omega})^{n} \cap V\right)$ непрерывно. Конструкция такого оператора приведена в [30]. Необходимость введения оператора регуляризации связана с тем, что в задачах гидродинамики вектор-функция скорости $v$ принадлежит $L_{2}(0, T ; V)$ и определение траектории $z(\tau ; t, x)$ возможно лишь для регуляризованного поля скоростей.

Тогда для каждого $v \in L_{2}(0, T ; V)$ уравнение $(5.51)$ имеет единственное решение $Z_{\delta}(v)$.

Итак, из (5.51) имеем $z(\tau ; t, x)=Z_{\delta}(\tau ; t, x)$. Эта функция и участвует в уравнении (5.48). 
Отметим также, что при равенстве константы $\mu_{1}$ нулю система (5.48)-(5.50) переходит в систему Навье-Стокса.

Разрешимость в слабом смысле начально-краевой задачи для регуляризованной модели движения жидких сред с памятью установлена в работе [31] и подробно описана в [32].

Отметим, что в данной модели существование аттракторов удается доказать не при всех значениях входящих параметров, а потребовалось найти соотношения между параметрами, при которых и установлено существование аттракторов для данной модели.

Обозначим через $V_{\alpha}, \alpha \in(0,1]$, замыкание в норме $W_{2}^{\alpha}$ множества $\mathscr{V}$ гладких соленоидальных функций с компактным носителем, содержащимся в $\Omega$. Пусть $V_{\alpha}^{*}$ - пространство, сопряженное к $V_{\alpha}$. С учетом отождествления $H \equiv H^{*}$ имеет место компактное вложение $H \subset V_{\alpha}^{*}$.

В качестве пространства траекторий $\mathscr{H}^{+}$задачи (5.48)-(5.50) возьмем множество функций $v \in L_{\infty}\left(\mathbb{R}_{+} ; H\right) \cap L_{2}^{\text {loc }}\left(\mathbb{R}_{+} ; V\right)$ с производной $v^{\prime} \in L_{1}^{\text {loc }}\left(\mathbb{R}_{+} ; V^{*}\right)$ таких, что ограничение $v$ на любой отрезок $[0, T]$ является слабым решением задачи (5.48)-(5.50) и при любом $t \geqslant 0$ выполняются неравенства

$$
\begin{aligned}
& \underset{s \in[t, t+1]}{\operatorname{vraimax}}\|v(s)\|_{H} \leqslant C_{1}\left(1+\|v\|_{L_{\infty}\left(\mathbb{R}_{+} ; H\right)}^{2} e^{-2 \gamma t}\right)^{1 / 2}, \\
& \int_{0}^{t} e^{-2 \gamma(t-s)}\|v(s)\|_{V}^{2} d s \leqslant C_{1}^{2}\left(1+\|v\|_{L_{\infty}\left(\mathbb{R}_{+} ; H\right)}^{2} e^{-2 \gamma t}\right)
\end{aligned}
$$

с некоторыми постоянными $C_{1}>0$ и $\gamma>0$, не зависящими от $v$.

Отметим, что введенное пространство содержится в классе $C\left(\mathbb{R}_{+} ; V_{\alpha}^{*}\right) \cap$ $L_{\infty}\left(\mathbb{R}_{+} ; H\right)$ и непусто (более того, из каждой точки пространства $H$ выходит по крайней мере одна траектория).

Отметим любопытную особенность пространства траекторий системы с памятью, отличающую ее от пространств траекторий, введенных для других задач гидродинамики. В определении других пространств траекторий участвовали оценки значений траекторий и их производных в точке или на отрезке фиксированной длины. Напротив, величина, стоящая в левой части неравенства (5.53), учитывает всю предысторию течения с начального момента. Однако наличие экспоненты под знаком интеграла говорит о том, что в основном эта величина все же определяется сравнительно недавней историей.

Имеют место следующие теоремы [33].

Теорема 5.9. Предположим, что параметры $\mu_{0}, \mu_{1}, \lambda$ задачи (5.48)-(5.50) связаны соотношением $\mu_{0}-\mu_{1} \lambda>0$, и пусть $\varphi \in V^{*}$. Тогда существует минимальный траекторный аттрактор $\mathscr{U}$ пространства траекторий $\mathscr{H}^{+}$задачи (5.48)-(5.50). Аттрактор ограничен в $L_{\infty}\left(\mathbb{R}_{+} ; H\right)$, компактен в $C\left(\mathbb{R}_{+} ; V_{\alpha}^{*}\right)$; он притягивает в топологии пространства $C\left(\mathbb{R}_{+} ; V_{\alpha}^{*}\right)$ ограниченные в норме $L_{\infty}\left(\mathbb{R}_{+} ; H\right)$ множества траекторий. 
Теорема 5.10. Предположим, что параметры $\mu_{0}, \mu_{1}, \lambda$ задачи (5.48)-(5.50) связаны соотношением $\mu_{0}-\mu_{1} \lambda>0$, и путь $\varphi \in V^{*}$. Тогда существует глобальный аттрактор $\mathscr{A}$ пространства траекторий $\mathscr{H}^{+}$задачи (5.48)-(5.50). Aттрактор ограничен в $H$, компактен в $V_{\alpha}$; он притягивает в топологии пространства $V_{\alpha}^{*}$ ограниченные в норме $L_{\infty}\left(\mathbb{R}_{+} ; H\right)$ множсества траекторий.

Согласно общей теории (теоремы 4.1 и 4.2), для доказательства этих теорем достаточно построить полуаттрактор пространства траекторий $\mathscr{H}^{+}$. Прежде чем построить такой полуаттрактор, приведем следующую оценку производной траектории по времени.

Лемма 5.3. Пусть $v \in \mathscr{H}^{+}$. Тогда $v^{\prime} \in L_{4 / 3}^{\text {loc }}\left(\mathbb{R}_{+} ; V^{*}\right)$ u nрu $t \geqslant 0$ имеет место неравенство

$$
\left\|v^{\prime}\right\|_{L_{4 / 3}\left(t, t+1 ; V^{*}\right)} \leqslant \mathscr{M}\left(I_{1}(t), I_{2}(t)\right)
$$

əдe

$$
\begin{aligned}
& I_{1}(t)=\underset{s \in[t, t+1]}{\operatorname{vraimax}}\|v(s)\|_{H}, \\
& I_{2}(t)=\max _{\tau \in[t, t+1]} \int_{0}^{\tau} e^{-2 \gamma(\tau-s)}\|v(s)\|_{V}^{2} d s,
\end{aligned}
$$

а аргументов, причем $\mathscr{M}\left(I_{1}, I_{2}\right)$ не убъвает по $I_{1}$ при фиксированном $I_{2}$ и по $I_{2}$ при фиксированном $I_{1}$.

Положим

$$
C_{2}=\mathscr{M}\left(2 C_{1}, 4 C_{1}^{2}\right) .
$$

В качестве возможного полуаттрактора можно рассмотреть множество

$$
\begin{aligned}
P=\{ & v \in L_{\infty}\left(\mathbb{R}_{+} ; H\right) \cap C\left(\mathbb{R}_{+} ; V_{\alpha}^{*}\right): \\
& \left.\forall t \geqslant 0\|v\|_{L_{\infty}(t, t+1 ; H)}+\left\|v^{\prime}\right\|_{L_{4 / 3}\left(t, t+1 ; V^{*}\right)} \leqslant 2 C_{1}+C_{2}\right\} .
\end{aligned}
$$

Ограниченность множества $P$ в норме $L_{\infty}\left(\mathbb{R}_{+} ; H\right)$ и его компактность в пространстве $C\left(\mathbb{R}_{+} ; V_{\alpha}^{*}\right)$, а также его трансляционная инвариантность доказываются аналогично тому, как делалось, например, в модели Джеффриса.

Покажем, что $P$ - поглощающее множество. Пусть $B \subset \mathscr{H}^{+}-$множество, ограниченное в норме пространства $L_{\infty}\left(\mathbb{R}_{+} ; H\right) ;$ скажем, $\|v\|_{L_{\infty}\left(\mathbb{R}_{+} ; H\right)} \leqslant R$ для $v \in B$. Выберем число $t_{0}$ так, чтобы

$$
1+R^{2} e^{-2 \gamma t_{0}} \leqslant 4
$$

Пусть $h \geqslant t_{0}$. Для $v \in B$ с помощью оценки (5.52) получаем

$$
\begin{aligned}
\|\mathrm{T}(h) v\|_{L_{\infty}(t, t+1 ; H)} & =\|v\|_{L_{\infty}(t+h, t+h+1 ; H)} \\
& \leqslant C_{1}\left(1+\|v\|_{L_{\infty}\left(\mathbb{R}_{+} ; H\right)}^{2} e^{-2 \gamma(t+h)}\right)^{1 / 2} \\
& \leqslant C_{1}\left(1+R^{2} e^{-2 \gamma t_{0}}\right)^{1 / 2} \leqslant 2 C_{1} .
\end{aligned}
$$


Оценим производную с помощью леммы 5.3:

$$
\begin{aligned}
\left\|\mathrm{T}(h) v^{\prime}\right\|_{L_{4 / 3}\left(t, t+1 ; V^{*}\right)} & =\left\|v^{\prime}\right\|_{L_{4 / 3}\left(t+h, t+h+1 ; V^{*}\right)} \\
& \leqslant \mathscr{M}\left(I_{1}(t+h), I_{2}(t+h)\right) .
\end{aligned}
$$

Оценим $I_{1}(t+h)$ и $I_{2}(t+h)$ с помощью неравенств (5.52) и (5.53):

$$
\begin{aligned}
I_{1}(t+h) & =\operatorname{vraimax}_{s \in[t+h, t+h+1]}\|v(s)\|_{H} \leqslant C_{1}\left(1+R^{2} e^{-2 \gamma(t+h)}\right)^{1 / 2} \leqslant 2 C_{1} \\
I_{2}(t+h) & =\max _{\tau \in[t+h, t+h+1]} \int_{0}^{\tau} e^{-2 \gamma(\tau-s)}\|v(s)\|_{V}^{2} d s \\
& \leqslant C_{1}^{2} \max _{\tau \in[t+h, t+h+1]}\left(1+R^{2} e^{-2 \gamma \tau}\right)=C_{1}^{2}\left(1+R^{2} e^{-2 \gamma(t+h)}\right) \\
& \leqslant C_{1}^{2}\left(1+R^{2} e^{-2 \gamma t_{0}}\right)=4 C_{1}^{2} .
\end{aligned}
$$

Учитывая монотонность функции $\mathscr{M}$, из оценки (5.56) получаем

$$
\left\|\mathrm{T}(h) v^{\prime}\right\|_{L_{4 / 3}\left(t, t+1 ; V^{*}\right)} \leqslant \mathscr{M}\left(2 C_{1}, 4 C_{1}^{2}\right)=C_{2} .
$$

Из этого неравенства и из (5.55) следует, что

$$
\|\mathrm{T}(h) v\|_{L_{\infty}(t, t+1 ; H)}+\left\|\mathrm{T}(h) v^{\prime}\right\|_{L_{4 / 3}\left(t, t+1 ; V^{*}\right)} \leqslant 2 C_{1}+C_{2},
$$

т. е. $\mathrm{T}(h) v \in P$ при $h \geqslant t_{0}$. Мы доказали, что $P$ - поглощающее, а следовательно, притягивающее множество и траекторный полуаттрактор.

\section{6. Равномерные аттракторы неавтономных систем}

\section{1. Равномерные аттракторы семейства траекторных пространств.} Автономные уравнения описывают процессы, закон эволюции которых не меняется со временем. Однако встречаются ситуации, когда закон эволюции меняется. Соответствующие математические модели включают коэффициенты или операторы, зависящие от времени, т. е. эволюция описывается неавтономными уравнениями. Изучение аттракторов неавтономных систем представляет математический интерес [34].

Теория траекторных аттракторов может применяться и для изучения неавтономных уравнений. В неавтономном случае рассматривают так называемые равномерные аттракторы системы траекторных пространств. Далее мы убедимся, что такие аттракторы можно свести к обычным аттракторам неинвариантного траекторного пространства.

В работе [4] описана теория равномерных аттракторов семейства трансляционно инвариантных пространств траекторий. На основании этой теории установлено существование аттракторов неавтономной трехмерной системы Навье-Стокса. Теория равномерных аттракторов была развита в работе [12] для неинвариантных пространств траекторий. Также в работе [12] удалось освободиться от некоторых ограничений работы [4]: так, не требуется, чтобы пространство символов системы было полным метрическим пространством и чтобы пространства траекторий были замкнуты в каком-либо смысле. Эта последняя теория и будет описана в настоящем разделе. В качестве приложений будут 
исследованы равномерные аттракторы начально-краевой задачи для системы Джеффриса с объективной производной [17].

Рассмотрим два банаховых пространства $E \subset E_{0}$ (вложение непрерывно), пространство $E$ рефлексивно. Кроме того, введем некоторое множество $\mathscr{X}$ и предположим, что каждому $\sigma$ из некоторого непустого множества $\Sigma \subset \mathscr{X}$ поставлено в соответствие траекторное пространство

$$
\mathscr{H}_{\sigma}^{+} \subset C\left(\mathbb{R}_{+} ; E_{0}\right) \cap L_{\infty}\left(\mathbb{R}_{+} ; E\right) .
$$

В этом случае говорят, что задано семейство траекторных пространств $\left\{\mathscr{H}_{\sigma}^{+}\right.$: $\sigma \in \Sigma\}$. Множество $\Sigma$ называется пространством символов, а его элементы символами. Множество

$$
\mathscr{H}_{\Sigma}^{+}=\bigcup_{\sigma \in \Sigma} \mathscr{H}_{\sigma}^{+}
$$

называют облединенным пространством траекторий семейства $\left\{\mathscr{H}_{\sigma}^{+}: \sigma \in \Sigma\right\}$. Каждое из множеств $\mathscr{H}_{\sigma}^{+}$непусто, поэтому и множество $\mathscr{H}_{\Sigma}^{+}$непусто и, следовательно, может рассматриваться как траекторное пространство.

Дадим основные определения.

ОПРЕДЕЛЕНИЕ 6.1. Множество $P \subset C\left(\mathbb{R}_{+} ; E_{0}\right) \cap L_{\infty}\left(\mathbb{R}_{+} ; E\right)$ называется равномерно (относительно $\sigma \in \Sigma$ ) притягивающим (для семейства пространств $\left\{\mathscr{H}_{\sigma}^{+}: \sigma \in \Sigma\right\}$ ), если для всякого множества $B \subset \mathscr{H}_{\Sigma}^{+}$, ограниченного в пространстве $L_{\infty}\left(\mathbb{R}_{+} ; E\right)$, выполняется условие

$$
\sup _{u \in B} \inf _{v \in P}\|\mathrm{~T}(h) u-v\|_{C\left(\mathbb{R}_{+} ; E_{0}\right)} \rightarrow 0 \quad(h \rightarrow \infty) .
$$

ОПРЕДЕЛЕНИЕ 6.2. Множество $P \subset C\left(\mathbb{R}_{+} ; E_{0}\right) \cap L_{\infty}\left(\mathbb{R}_{+} ; E\right)$ называется равномерно поглощающим, если для всякого множества $B \subset \mathscr{H}_{\Sigma}^{+}$, ограниченного в пространстве $L_{\infty}\left(\mathbb{R}_{+} ; E\right)$, существует $h \geqslant 0$ такое, что при всех $t \geqslant h$ имеет место включение

$$
\mathrm{T}(t) B \subset P .
$$

Очевидно, что любое равномерно поглощающее множество является равномерно притягивающим.

ОПРЕДЕЛЕНИЕ 6.3. Множество $P \subset C\left(\mathbb{R}_{+} ; E_{0}\right) \cap L_{\infty}\left(\mathbb{R}_{+} ; E\right)$ называется равномерным траекторным полуаттрактором (семейства пространств $\left\{\mathscr{H}_{\sigma}^{+}\right.$: $\sigma \in \Sigma\}$ ), если оно удовлетворяет следующим условиям:

(i) множество $P$ компактно в $C\left(\mathbb{R}_{+} ; E_{0}\right)$ и ограничено в $L_{\infty}\left(\mathbb{R}_{+} ; E\right)$;

(ii) имеет место включение $\mathrm{T}(t) P \subset P$ для всех $t \geqslant 0$;

(iii) множество $P$ является равномерно притягивающим в смысле определения 6.1 . 
ОПРЕДЕЛЕНИЕ 6.4. Множество $P \subset C\left(\mathbb{R}_{+} ; E_{0}\right) \cap L_{\infty}\left(\mathbb{R}_{+} ; E\right)$ называется равномерным траекторным квазиаттрактором (семейства пространств $\left\{\mathscr{H}_{\sigma}^{+}: \sigma \in \Sigma\right\}$ ), если оно удовлетворяет условиям (i), (iii) определения 6.3, а также условию

(ii') имеет место включение $\mathrm{T}(t) P \supset P$ для всех $t \geqslant 0$.

ОПРЕДЕЛЕниЕ 6.5. Множество $P \subset C\left(\mathbb{R}_{+} ; E_{0}\right) \cap L_{\infty}\left(\mathbb{R}_{+} ; E\right)$ называется равномерным траекторным аттрактором семейства пространств $\left\{\mathscr{H}_{\sigma}^{+}: \sigma \in \Sigma\right\}$, если оно одновременно является и траекторным полуаттрактором, и траекторным квазиаттрактором, т. е. если оно удовлетворяет условиям (i), (iii) определения 6.3 , а также условию

$\left(\right.$ ii $\left.^{\prime \prime}\right)$ имеет место равенство $\mathrm{T}(t) P=P$ для всех $t \geqslant 0$.

ОПРЕДЕЛЕНИЕ 6.6. Минимальным траекторным аттрактором пространства траекторий $\mathscr{H}_{\Sigma}^{+}$называется наименьший по включению траекторный аттрактор, т. е. такой траекторный аттрактор, который содержится в любом другом траекторном аттракторе.

ОПРЕДЕЛЕНИЕ 6.7. Множество $\mathscr{A} \subset E$ называется равномерным (относительно $\sigma \in \Sigma$ ) глобальным аттрактором (в $\left.E_{0}\right)$ семейства пространств $\left\{\mathscr{H}_{\sigma}^{+}\right.$: $\sigma \in \Sigma\}$, если оно удовлетворяет следующим условиям:

(i) множество $\mathscr{A}$ компактно в $E_{0}$ и ограничено в $E$;

(ii) для всякого ограниченного в $L_{\infty}\left(\mathbb{R}_{+} ; E\right)$ множества $B \subset \mathscr{H}_{\Sigma}^{+}$выполняется условие притяжения

$$
\sup _{u \in B} \inf _{y \in \mathscr{A}}\|u(t)-y\|_{E_{0}} \rightarrow 0 \quad(t \rightarrow \infty)
$$

(iii) множество $\mathscr{A}$ является является наименьшим по включению, удовлетворяющим условиям (i) и (ii) (т. е. $\mathscr{A}$ содержится в каждом множестве, удовлетворяющем этим условиям).

ЗАмЕчАНИЕ 6.1. Очевидно, что если семейство траекторных пространств имеет минимальный траекторный аттрактор, то он единственный; если имеет глобальный аттрактор, то он также единственный.

Сравнив данные выше определения с определениями 4.1-4.5 аттракторов пространства траекторий, можно сделать вывод, что равномерный глобальный аттрактор и все виды равномерных траекторных аттракторов можно рассматривать как соответствующие аттракторы траекторного пространства $\mathscr{H}_{\Sigma}^{+}$; то же верно и относительно притягивающих и поглощающих множеств. В частности, определения $6.5,6.6$ и 6.7 можно переформулировать следующим образом.

ОПРЕДЕЛЕНИЕ 6.8. (Минималъным) равномерным траекторным аттрактором семейства $\left\{\mathscr{H}_{\sigma}^{+}: \sigma \in \Sigma\right\}$ называется (минимальный) траекторный аттрактор пространства $\mathscr{H}_{\Sigma}^{+}$.

ОПРЕДЕЛЕНИЕ 6.9. Глобалъным аттрактором семейства $\left\{\mathscr{H}_{\sigma}^{+}: \sigma \in \Sigma\right\}$ называется глобальный аттрактор пространства $\mathscr{H}_{\Sigma}^{+}$. 
Из определений 6.8 и 6.9 явствует, что принципиальной разницы между равномерными аттракторами и обычными аттракторами пространства траекторий нет. Отличие равномерных аттракторов состоит только в том, что траекторное пространство $\mathscr{H}_{\Sigma}^{+}$задается в виде объединения некоторого семейства траекторных пространств. Такой способ построения обусловлен приложениями данной теории.

Первоначально теория равномерных аттракторов семейств траекторных пространств мыслилась как обобщение теории аттракторов пространств траекторий (см. работу М. И. Вишика и В. В. Чепыжова [4] и их же монографию [6]). С переходом к рассмотрению трансляционно неинвариантных пространств траекторий оказалось возможным включить первую теорию во вторую.

Из определений 6.8 и 6.9 следует, что теоремы о существовании и свойствах аттракторов пространства траекторий автоматически переносятся на случай равномерных аттракторов. В частности, имеют место следующие теоремы существования.

ТЕОРЕма 6.1. Пусть существует равномерный траекторный полуаттрактор $P$ семейства траекторных пространств $\left\{\mathscr{H}_{\sigma}^{+}: \sigma \in \Sigma\right\}$. Тогда существует минимальный равномерный траекторный аттрактор $\mathscr{U}$ семейства траекторных пространств $\left\{\mathscr{H}_{\sigma}^{+}: \sigma \in \Sigma\right\}$, имеют место соотношения

$$
\Pi_{+} \mathscr{K}\left(\mathscr{H}_{\Sigma}^{+}\right) \subset \mathscr{U}=\Pi_{+} \mathscr{K}(\mathscr{U}) \subset \Pi_{+} \mathscr{K}(P) \subset P
$$

и ядро $\mathscr{K}\left(\mathscr{H}^{+}\right)$является относительно компактным в $C\left(\mathbb{R} ; E_{0}\right)$ и ограниченным в норме $L_{\infty}(\mathbb{R} ; E)$.

ТЕОРема 6.2. Пусть существует минимальный равномерный траекторный аттрактор $\mathscr{U}$ семейства траекторных пространств $\left\{\mathscr{H}_{\sigma}^{+}: \sigma \in \Sigma\right\}$. Тогда существует равномерный глобальный аттрактор $\mathscr{A}$ семейства траекторных пространств $\left\{\mathscr{H}_{\sigma}^{+}: \sigma \in \Sigma\right\}$ и справедливы соотношения

$$
\begin{aligned}
\mathscr{A} & =\mathscr{U}(t), & & t \geqslant 0 ; \\
\mathscr{K}\left(\mathscr{H}_{\Sigma}^{+}\right)(t) \subset \mathscr{A} & =\mathscr{K}(\mathscr{U})(t), & & t \in \mathbb{R} .
\end{aligned}
$$

Разумеется, для равномерных аттракторов верны и другие результаты раздела 4.

Опишем теперь обычную схему применения семейств траекторных пространств для изучения неавтономных уравнений.

Пусть имеется неавтономное эволюционное уравнение вида

$$
v^{\prime}(t)=A_{\sigma_{0}(t)}(v(t))
$$

где $A_{\sigma_{0}(t)}$ - некий оператор, зависящий от времени $t$ через функцию $\sigma_{0}$, называемую символом уравнения. В приложениях $\sigma_{0}$ является совокупностью всех зависящих от $t$ членов уравнения (6.3) и представляет собой функцию, определенную (всюду или почти всюду) на $\mathbb{R}_{+}$и принимающую значение в некотором банаховом пространстве. Класс зависящих от времени функций, к которому 
принадлежит символ $\sigma_{0}$, обозначим $\mathscr{X}$ (обычно $\mathscr{X}$ - топологическое векторное пространство).

Наряду с исходным уравнением (6.3) рассмотрим семейство уравнений

$$
v^{\prime}(t)=A_{\sigma(t)}(v(t)) \quad(\sigma \in \Sigma),
$$

где $\Sigma$ - некоторое непустое подмножество пространства $\mathscr{X}$, называемое $n$ ространством символов уравнения (6.4) (элементы этого множества называются символами соответствующих уравнений).

Вообще говоря, пространство символов $\Sigma$ определяется по исходному символу $\sigma_{0}$, но выбор этого пространства неоднозначен и остается на усмотрении исследователя. Естественно, различным $\Sigma$ соответствуют различные семейства траекторных пространств и различные аттракторы. Разумно требовать, чтобы исходный символ $\sigma_{0}$ принадлежал $\Sigma$, и тогда наименьшее возможное пространство символов есть $\Sigma=\left\{\sigma_{0}\right\}$. Также можно потребовать, чтобы пространство символов содержало множество $\Sigma_{0}=\left\{\mathrm{T}(t) \sigma_{0}: t \geqslant 0\right\}$ (здесь $\mathrm{T}(t)$ - трансляционные операторы, которые действуют на $\mathscr{X})$, и, в частности, $\Sigma$ может совпадать с $\Sigma_{0}$ или с замыканием $\Sigma_{0}$ в сильной или слабой топологии пространства $\mathscr{X}$.

Для каждого $\sigma \in \Sigma$ определяется траекторное пространство $\mathscr{H}_{\sigma}^{+}$, состоящее из некоторых определенных на $\mathbb{R}_{+}$решений уравнения (6.4) (это пространство может выбираться с таким же произволом, как в автономном случае, т. е. используемое конкретное определение решения уравнения не имеет значения). Таким образом, получаем семейство траекторных пространств $\left\{\mathscr{H}_{\sigma}^{+}: \sigma \in \Sigma\right\}$, равномерные аттракторы которого и называются равномерными аттракторами уравнения (6.4).

6.2. Равномерные аттракторы системы Джеффриса с объективной производной. В качестве приложения теории равномерных аттракторов рассмотрим начально-краевую задачу для системы Джеффриса с объективной производной. Существование равномерных траекторного и глобального аттрактора для этой задачи было установлено в [17]. Сходным образом можно рассматривать и систему Джеффриса с полной производной [12].

6.2.1. Принцип объективности. Принцип объективности является одним из основных принципов рациональной механики. Говоря неформально, он утверждает, что вид реологических соотношений механики должен сохраняться при изменении системы отсчета.

Описание движения жидкости включает в себя некоторые тензоры (точнее говоря, тензорные функции точек пространства и времени). Нас особо интересуют тензор скоростей деформации $\mathscr{E}$ и девиатор тензора напряжений $\sigma$, поскольку они входят в рассматриваемые реологические соотношения. Если задана некоторая система отсчета (под которой мы понимаем прямоугольную систему координат в пространстве и часы), то такие тензоры задаются матрицами: например, компоненты тензора $\mathscr{E}$ выражаются формулами

$$
\mathscr{E}_{i j}=\frac{1}{2}\left(\frac{\partial v_{i}}{\partial x_{j}}+\frac{\partial v_{j}}{\partial x_{i}}\right) \quad(i, j=1,2,3) .
$$


Предположим, имеются две системы отсчета: одна - с координатами $x=$ $\left(x_{1}, x_{2}, x_{3}\right)$ и временем $t$, другая - с координатами $x^{*}=\left(x_{1}^{*}, x_{2}^{*}, x_{3}^{*}\right)$ и временем $t^{*}$. Наиболее общие соотношения, связывающие пространственно-временные координаты в обеих системах, имеют вид

$$
t^{*}=t+a, \quad x^{*}=x_{0}^{*}(t)+Q(t) x,
$$

где $a \in \mathbb{R}, x \in \mathbb{R}^{3}, Q(t) \in O(3)$. Если в одной и той же точке пространства вычислить компоненты тензора скоростей деформации по формулам (6.5) для координат $x$ и $x^{*}$, получатся матрицы $\mathscr{E}$ и $\mathscr{E} *$, связанные соотношением

$$
\mathscr{E}^{*}=Q \mathscr{E} Q^{\top}
$$

Если компоненты некоторой тензорной функции в разных системах отсчета связаны формулой

$$
T^{*}\left(t^{*}, x^{*}\right)=Q(t) T(t, x) Q(t)^{\top}
$$

говорят, что такая функция не зависит от наблюдателя. Например, тензоры $\mathscr{E}$ и $\sigma$ не зависят от наблюдателя.

В реологическое соотношение может входить производная тензорной функции по времени: например, частная $\frac{\partial}{\partial t}$ или полная $\frac{d}{d t}=\frac{\partial}{\partial t}+\sum_{i=1}^{3} v_{i} \frac{\partial}{\partial x_{i}}$. Когда тензор задан в координатах матрицей $T$, взятие производной по времени преобразует эту матрицу в новую матрицу $\dot{T}$. Может оказаться, что матрица $\dot{T}$ не преобразуется по закону (6.7), даже если исходный тензор не зависел от системы отсчета.

ОПредЕлЕниЕ 6.10. Пусть $T(t, x)$ - произвольная тензорнозначная функция, не зависящая от наблюдателя. Оператор вида

$$
\frac{D T(t, x)}{D t}=\frac{d T(t, x)}{d t}+G(\nabla v(t, x), T(t, x))
$$

где $G$ - некоторая матричнозначная функция двух матричных аргументов, называется обгективной производной, если при любом изменении системы отсчета (6.6) выполнено равенство

$$
\frac{D^{*} T^{*}}{D t^{*}}=Q(t) \frac{D T}{D t} Q(t)^{\top}
$$

для всех возможных функций $T$.

ЗАмечАниЕ 6.2 . Символ $\frac{D^{*}}{D t^{*}}$ обозначает представление оператора $\frac{D}{D t}$ в новой системе отсчета, т. е. выражение

$$
\frac{d T^{*}}{d t^{*}}+G\left(\nabla^{*} v^{*}, T^{*}\right)=\frac{\partial T^{*}}{\partial t^{*}}+\sum_{i=1}^{3} v_{i}^{*} \frac{\partial T^{*}}{\partial x_{i}^{*}}+G\left(\nabla^{*} v^{*}, T^{*}\right)
$$


Выбор функции $G$ осуществляется на основе различных механических и опытных соображений.

Ни частная, ни полная производная не являются объективными.

Самый простой пример объективной производной тензора - это производная Яуманна, или коротационная:

$$
\frac{D_{0} T(t, x)}{D t}=\frac{d T(t, x)}{d t}+T(t, x) W(t, x)-W(t, x) T(t, x),
$$

где $W=\left(W_{i j}\right)$ - тензор завихрености, компоненты которого определяются формулами

$$
W_{i j}=\frac{1}{2}\left(\frac{\partial v_{i}}{\partial x_{j}}-\frac{\partial v_{j}}{\partial x_{i}}\right) .
$$

Рассмотрим еще один пример объективной производной, с которой будем работать дальше. Это производная, полученная регуляризацией производной Яуманна: тензор завихренности, входящий в определение этой производной, заменяется своим осреднением.

Осреднение заключается в следующем. Пусть $\rho: \mathbb{R}^{n} \rightarrow \mathbb{R}$ - неотрицательная гладкая функция с компактным носителем такая, что $\rho(x)=\rho(y)$ при $|x|=|y|$ и выполнено равенство

$$
\int_{\mathbb{R}^{n}} \rho(y) d y=1
$$

Предположим, что тензор завихренности $W$ доопределен нулем вне области $\Omega$, занятой средой. Осреднение определяется формулой

$$
W_{\rho}(v)(t, x)=\int_{\mathbb{R}^{n}} \rho(x-y) W(v)(t, y) d y .
$$

Отметим, что интеграл в правой части фактически берется по ограниченной области.

Осреднение имеет смысл для суммируемого тензора завихренности, что заведомо будет иметь место, если поле скоростей принадлежит пространству $V$ соленоидальных функций, первые производные которых суммируемы в $\Omega$. Осреднение является аппроксимацией тензора завихренности, гладкой по пространственным переменным.

Мы будем рассматривать производную

$$
\frac{d T}{d t}+T W_{\rho}-W_{\rho} T
$$

близкую к производной Яуманна. Эта производная также объективна.

6.2.2. Система Джеффриса с объективной производной. Пусть $\Omega$ - ограниченная область с кусочно липшицевой границей в пространстве $\mathbb{R}^{n}$, где $n=2,3$. 
Рассмотрим начально-краевую задачу

$$
\begin{aligned}
& \frac{\partial v}{\partial t}+\sum_{i=1}^{n} v_{i} \frac{\partial v}{\partial x_{i}}+\operatorname{grad} p=\operatorname{Div} \sigma+f \\
& \sigma+\lambda_{1}\left(\frac{\partial \sigma}{\partial t}+\sum_{i=1}^{n} v_{i} \frac{\partial \sigma}{\partial x_{i}}+\sigma W_{\rho}-W_{\rho} \sigma\right) \\
& \quad=2 \eta\left(\mathscr{E}+\lambda_{2}\left(\frac{\partial \mathscr{E}}{\partial t}+\sum_{i=1}^{n} v_{i} \frac{\partial \mathscr{E}}{\partial x_{i}}+\mathscr{E} W_{\rho}-W_{\rho} \mathscr{E}\right)\right), \\
& \operatorname{div} v=0, \\
& \left.v\right|_{\partial \Omega}=0, \\
& \left.v\right|_{t=0}=a,\left.\quad \sigma\right|_{t=0}=\sigma_{0} .
\end{aligned}
$$

Здесь $v$ - неизвестное поле скоростей среды, $p$ - неизвестная функция давления, $\sigma$ - неизвестный девиатор тензора напряжений, $f$ - известная плотность внешних сил (все эти величины зависят от точки $x \in \Omega$ и момента времени $t$ ), $\mathscr{E}-$ тензор скоростей деформации, $W_{\rho}$ - регуляризованный тензор завихренности, описанный в предыдущем пункте, $\eta$ - вязкость среды, $\lambda_{1}$ - время релаксации, $\lambda_{2}$ - время ретардации: $\eta, \lambda_{1}, \lambda_{2}$ - положительные постоянные, причем $\lambda_{1}>\lambda_{2}$; наконец, $a$ и $\sigma_{0}-$ заданные функции.

В задаче (6.13)-(6.17) уравнение (6.13) является общим уравнением движения, уравнение (6.14) - реологическим соотношением Джеффриса (5.4) с производной (6.12), уравнение (6.15) - уравнением непрерывности, уравнение (6.16) выражает условие прилипания, а (6.17) - начальные условия. Выше было показано, что производная (6.12) объективна, поэтому реологическое соотношение (6.14) объективно, т. е. не меняет своего вида при замене переменных (6.6).

Будем использовать те же функциональные пространства, что и для системы Джеффриса с полной производной.

Как и при рассмотрении системы с полной производной, введем новые обозначения:

$$
\mu_{1}=\frac{\eta \lambda_{2}}{\lambda_{1}}, \quad \mu_{2}=\frac{\eta-\mu_{1}}{\lambda_{1}} \quad\left(\mu_{1}, \mu_{2}>0\right) \quad \text { и } \quad \tau=\sigma-2 \mu_{1} \mathscr{E}(v) .
$$

Таким образом, от неизвестных $(v, \sigma)$ мы переходим к неизвестным $(v, \tau)$, где $\tau$ - симметрическая матрица порядка $n$ (зависящая от $x, t$ ). Ищутся пары функций $(v, \tau)$ классов

$$
\left.\begin{array}{rl}
v & \in L_{2}(0, T ; V) \cap L_{\infty}(0, T ; H) \cap C_{w}([0, T] ; H), \\
v^{\prime} & \in L_{1}\left(0, T ; V^{*}\right) ; \\
\tau & \in L_{\infty}\left(0, T ; L_{2}\right) \cap C_{w}\left([0, T] ; L_{2}\right), \\
\tau^{\prime} & \in L_{2}\left(0, T ; H^{-2}\right),
\end{array}\right\}
$$


которые удовлетворяют интегральным тождествам

$$
\begin{gathered}
\frac{d}{d t}(\tau, \Phi)+\frac{1}{\lambda_{1}}(\tau, \Phi)-\sum_{i=1}^{n}\left(v_{i} \tau, \frac{\partial \Phi}{\partial x_{i}}\right) \\
+2 \mu_{2}(v, \operatorname{Div} \Phi)+\left(\tau W_{\rho}-W_{\rho} \tau, \Phi\right)=0, \\
\frac{d}{d t}(v, \varphi)-\sum_{i=1}^{n}\left(v_{i} v, \frac{\partial \varphi}{\partial x_{i}}\right)+\mu_{1}(\nabla v, \nabla \varphi)+(\tau, \nabla \varphi)=\langle f, \varphi\rangle
\end{gathered}
$$

для всех $\varphi \in \mathscr{V}$ и $\Phi \in C_{0}^{\infty}$ почти всюду на $(0, T)$.

Уравнения, определяющие $(v, \tau)$, снабдим начальными условиями

$$
v(0)=a, \quad \tau(0)=\tau_{0} .
$$

Сформулируем теорему существования решений системы (6.19)-(6.21), включив в нее энергетическую оценку диссипативного типа, на которой основывается существование аттракторов (см. [17]).

TeOpema 6.3. Пусть $f \in L_{2}\left(0, T ; V^{*}\right), a \in H, \tau_{0} \in L_{2}\left(\Omega, M_{S}(n)\right)$. Тогда на отрезке $[0, T]$ существует решение $(v, \tau)$ задачи (6.19)-(6.21), удовлетворяющее неравенству

$$
\begin{aligned}
& \frac{1}{4}\|v\|_{L_{\infty}(t, t+1 ; H)}^{2}+\frac{1}{8 \mu_{2}}\|\tau\|_{L_{\infty}\left(t, t+1 ; L_{2}\right)}^{2}+\frac{\mu_{1}}{2}\|v\|_{L_{2}(t, t+1 ; V)}^{2} \\
& \leqslant e^{-2 \gamma t}\left(\|a\|_{H}^{2}+\frac{1}{2 \mu_{2}}\left\|\tau_{0}\right\|_{L_{2}}^{2}\right) \\
& \quad+\frac{2 e^{4 \gamma}+e^{2 \gamma}-1}{2 \mu_{1}\left(e^{2 \gamma}-1\right)} \sup _{s \in[0, T-1]}\|f\|_{L_{2}\left(s, s+1 ; V^{*}\right)}^{2} \quad(t \in[0, T-1])
\end{aligned}
$$

с постоянной

$$
\gamma=\min \left\{\frac{1}{\lambda_{1}}, \frac{\mu_{1}}{2 K_{0}^{2}}\right\},
$$

где $K_{0}$ - постоянная из неравенств (5.15), (5.16).

Доказательство в общих чертах повторяет доказательство теоремы 5.3. Сначала неравенство (6.22) доказывается для решений некоторой аппроксимационной системы. Затем обосновывается предельный переход к решениям исходной системы.

Выразив из тождеств (6.19), (6.20) члены, содержащие производные, можно получить следующие априорные оценки производных.

Лемма 6.1. Пусть $f \in L_{2}\left(0, T ; V^{*}\right)$, и пусть пара функиий $(v, \tau)$ классов (6.18) удовлетворяет тождествам (6.19), (6.20) почти всюду на $(0, T)$ при любых $\varphi \in \mathscr{V}$ u $\Phi \in C_{0}^{\infty}$. Тогда для любого отрезка $\left[t_{1}, t_{2}\right] \subset[0, T]$ имеет место оиенка

$$
\begin{gathered}
\|v\|_{L_{4 / 3}\left(t_{1}, t_{2} ; V^{*}\right)}+\left\|\tau^{\prime}\right\|_{L_{2}\left(t_{1}, t_{2} ; H^{-2}\right)} \leqslant \mathscr{M}\left(\|v\|_{L_{\infty}\left(t_{1}, t_{2} ; H\right)},\|v\|_{L_{2}\left(t_{1}, t_{2} ; V\right)},\right. \\
\left.\|\tau\|_{L_{\infty}\left(t_{1}, t_{2} ; L_{2}\right)},\|f\|_{L_{2}\left(t_{1}, t_{2} ; V^{*}\right)}, t_{2}-t_{1}\right)
\end{gathered}
$$


где $\mathscr{M}$ - некоторая вещественная функиия, неубывающая по каждому из своих аргументов.

6.2.3. Равномерные аттракторы системы Джеффриса. Перейдем к рассмотрению равномерных аттракторов системы (6.19), (6.20), предполагая, что функция $f$ зависит от $t$ и определена почти всюду на $\mathbb{R}_{+}$. Для конечного отрезка существование решений доказано для плотности внешних сил, принадлежащей пространству $L_{2}\left(0, T ; V^{*}\right)$, поэтому при изучении аттракторов естественно считать $f$ принадлежащей пространству $L_{2}^{\text {loc }}\left(\mathbb{R}_{+} ; V^{*}\right)$.

В качестве пары банаховых пространств, требующихся для введения пространств траекторий, выберем $E=H \times L_{2}, E_{0}=V_{\alpha}^{*} \times H^{-\alpha}$.

В системе (6.19), (6.20) среди известных величин только функция $f$ зависит от времени, поэтому ее следует рассматривать в качестве символа. Следовательно, множество символов естественно рассматривать в пространстве $\mathscr{X}=L_{2}^{\text {loc }}\left(\mathbb{R}_{+} ; V^{*}\right)$. Пространство $\mathscr{X}$ банахово с нормой

$$
\|\varphi\|_{\mathscr{X}}=\sup _{t \geqslant 0}\|\varphi\|_{L_{2}\left(t, t+1 ; V^{*}\right)}
$$

Для фиксированного $f \in \mathscr{X}$ в качестве пространства символов $\Sigma$ системы (6.19), (6.20) можно выбрать одно из следующих:

1) $\Sigma=\{f\}$;

2) $\Sigma=\Sigma_{0}(f) \stackrel{\text { def }}{=}\{\mathrm{T}(t) f: t \geqslant 0\}$;

3) $\Sigma$ - замыкание множества $\Sigma_{0}$ в сильной топологии пространства $\mathscr{X}$;

4) $\Sigma$ - замыкание множества $\Sigma_{0}$ в слабой топологии пространства $\mathscr{X}$;

5) $\Sigma$ - произвольное множество в пространстве $\mathscr{X}$, содержащее $\Sigma_{0}$ и имеющее свойство

$$
\|\sigma\|_{\mathscr{X}}=\|f\|_{\mathscr{X}} \quad(\sigma \in \Sigma)
$$

6) $\Sigma$ - произвольное множество со свойством (6.24) в пространстве $\mathscr{X}$, содержащее функцию $f$.

Разному выбору множества $\Sigma$ отвечают, вообще говоря, разные равномерные аттракторы, но доказательство их существования одинаково для всех случаев. Каждый из приведенных случаев содержит предыдущий. Случай 1) соответствует наиболее простому решению, когда семейство траекторных пространств состоит из единственного пространства, образованного некоторыми слабыми решениями системы (6.19), (6.20). В случаях 2)-5) добавляются пространства траекторий, состоящие из решений системы (6.19), (6.20) с $f$, замененным на $\mathrm{T}(t) f(t \geqslant 0)$. Случай 6$)$ имеет весьма общий характер.

Предположим, что множество $\Sigma \subset \mathscr{X}$, удовлетворяющее самому слабому условию 6), выбрано и зафиксировано и для любого $\sigma \in \Sigma$ выполняется равенство (6.24). Определим семейство траекторных пространств $\left\{\mathscr{H}_{\sigma}^{+}: \sigma \in \Sigma\right\}$.

ОПРЕДЕЛЕНИЕ 6.11. Пространство траекторий $\mathscr{H}_{\sigma}^{+}$системы (6.19), (6.20), соответствующее символу $\sigma \in \Sigma$, - это множество пар функций $(v, \tau)$, удовлетворяющих следующим условиям: 
(i) функции $v$ и $\tau$ принадлежат классам

$$
v \in L_{2}^{\text {loc }}\left(\mathbb{R}_{+} ; V\right) \cap L_{\infty}\left(\mathbb{R}_{+} ; H\right), \quad \tau \in L_{\infty}\left(\mathbb{R}_{+} ; L_{2}\right) ;
$$

(ii) пара $(v, \tau)$ удовлетворяет равенству (6.19) и равенству

$$
\frac{d}{d t}(v, \varphi)-\sum_{i=1}^{n}\left(v_{i} v, \frac{\partial \varphi}{\partial x_{i}}\right)+\mu_{1}(\nabla v, \nabla \varphi)+(\tau, \nabla \varphi)=\langle\sigma, \varphi\rangle
$$

при всех $\varphi \in \mathscr{V}$ и $\Phi \in C_{0}^{\infty}$ для почти всех $t \geqslant 0$;

(iii) при всех $t \geqslant 0$ функции $v$ и $\tau$ удовлетворяют энергетическому неравенству

$$
\begin{aligned}
& \frac{1}{4}\|v\|_{L_{\infty}(t, t+1 ; H)}^{2}+\frac{1}{8 \mu_{2}}\|\tau\|_{L_{\infty}\left(t, t+1 ; L_{2}\right)}^{2}+\frac{\mu_{1}}{2}\|v\|_{L_{2}(t, t+1 ; V)}^{2} \\
& \quad \leqslant e^{-2 \gamma t}\left(\|v\|_{L_{\infty}\left(\mathbb{R}_{+} ; H\right)}^{2}+\frac{1}{2 \mu_{2}}\|\tau\|_{L_{\infty}\left(\mathbb{R}_{+} ; L_{2}\right)}^{2}\right)+\frac{2 e^{4 \gamma}+e^{2 \gamma}-1}{2 \mu_{1}\left(e^{2 \gamma}-1\right)}\|f\|_{\mathscr{X}}^{2}
\end{aligned}
$$

где $\gamma$ - постоянная из теоремы 6.3 .

Вложение

$$
\mathscr{H}_{\sigma}^{+} \subset C\left(\mathbb{R}_{+} ; E_{0}\right) \cap L_{\infty}\left(\mathbb{R}_{+} ; E\right)
$$

проверяется так же, как для системы Джеффриса с полной производной в автономном случае (п. 5.2.3).

Имеет место следующая теорема о существовании траекторий.

Теорема 6.4. Пусть $\sigma \in \Sigma-$ некоторый символ. Тогда для любых $a \in H$, $\tau_{0} \in L_{2}\left(\Omega, M_{S}(n)\right)$ существует траектория $(v, \tau) \in \mathscr{H}_{\sigma}^{+}$, для которой

$$
v(0)=a, \quad \tau(0)=\tau_{0} .
$$

ДокАзАтЕльство. Для заданного $\sigma \in \Sigma$ рассмотрим систему (6.19), (6.25), и пусть $\left(v_{m}, \tau_{m}\right)$ - существующее по теореме 6.3 решение этой системы с начальным условием $v_{m}(0)=a, \tau_{m}(0)=\tau_{0}$ на отрезке $\left[0, T_{m}\right]$, где $\left\{T_{m}\right\}-$ некоторая последовательность, возрастающая и стремящаяся к $\infty$. Эти решения продолжаются на полуось $\mathbb{R}_{+}$до функций $\widetilde{v}_{m}, \widetilde{\tau}_{m} ;$ без ограничения общности можно считать, что эти продолжения в некотором смысле сходятся к паре функций $(v, \tau)$, которая является решением системы $(6.19),(6.25)$ на $\mathbb{R}_{+}$и удовлетворяет начальным условиям (6.27); в частности,

$$
\widetilde{v}_{m} \rightarrow v \quad \text { слабо в } L_{2}(0, T ; V) \text { и } * \text {-слабо в } L_{\infty}(0, T ; H)
$$

и

$$
\widetilde{\tau}_{m} \rightarrow \tau \quad \text { *-слабо в } L_{\infty}\left(0, T ; L_{2}\right)
$$

при любом $T>0$. 
По теореме 6.3 решения $\left(v_{m}, \tau_{m}\right)$ на любом отрезке $[0, T]$ (где они определены) удовлетворяют неравенству

$$
\begin{gathered}
\frac{1}{4}\left\|v_{m}\right\|_{L_{\infty}(t, t+1 ; H)}^{2}+\frac{1}{8 \mu_{2}}\left\|\tau_{m}\right\|_{L_{\infty}\left(t, t+1 ; L_{2}\right)}^{2}+\frac{\mu_{1}}{2}\left\|v_{m}\right\|_{L_{2}(t, t+1 ; V)}^{2} \\
\leqslant e^{-2 \gamma t}\left(\|a\|_{H}^{2}+\frac{1}{2 \mu_{2}}\left\|\tau_{0}\right\|_{L_{2}}^{2}\right)+\frac{2 e^{4 \gamma}+e^{2 \gamma}-1}{2 \mu_{1}\left(e^{2 \gamma}-1\right)}\|\sigma\|_{\mathscr{X}}^{2} .
\end{gathered}
$$

Так же как и в доказательстве теоремы 5.4, переходим к пределу и за счет свойств слабой сходимости для предельных функций $v, \tau$ получаем неравенство

$$
\begin{gathered}
\frac{1}{4}\|v\|_{L_{\infty}(t, t+1 ; H)}^{2}+\frac{1}{8 \mu_{2}}\|\tau\|_{L_{\infty}\left(t, t+1 ; L_{2}\right)}^{2}+\frac{\mu_{1}}{2}\|v\|_{L_{2}(t, t+1 ; V)}^{2} \\
\leqslant e^{-2 \gamma t}\left(\|a\|_{H}^{2}+\frac{1}{2 \mu_{2}}\left\|\tau_{0}\right\|_{L_{2}}^{2}\right)+\frac{2 e^{4 \gamma}+e^{2 \gamma}-1}{2 \mu_{1}\left(e^{2 \gamma}-1\right)}\|\sigma\|_{\mathscr{X}}^{2}
\end{gathered}
$$

которое выполняется для всех $t \in \mathbb{R}_{+}$. Для того чтобы получить свойство (iii) определения 6.11 , остается только оценить левую часть этого неравенства величиной

$$
e^{-2 \gamma t}\left(\|v\|_{L_{\infty}\left(\mathbb{R}_{+} ; H\right)}+\frac{1}{2 \mu_{2}}\left\|\tau_{0}\right\|_{L_{\infty}\left(\mathbb{R}_{+} ; L_{2}\right)}^{2}\right)+\frac{2 e^{4 \gamma}+e^{2 \gamma}-1}{2 \mu_{1}\left(e^{2 \gamma}-1\right)}\|f\|_{\mathscr{X}}^{2},
$$

учитывая, что для $\sigma \in \Sigma$ выполняется неравенство $\|\sigma\|_{\mathscr{X}} \leqslant\|f\|_{\mathscr{X}}$. Теорема 6.4 доказана.

Теорема 6.5 [17]. Пусть в правой части уравнения (6.20) функиия $f$ является элементом пространства $\mathscr{X}$, и пусть множество символов $\Sigma \subset \mathscr{X}$, содержащее функиию $f$, таково, что для каждого $\sigma \in \Sigma$ выполняется условие $\|\sigma\|_{\mathscr{X}} \leqslant\|f\|_{\mathscr{X}}$. Тогда существует минимальный равномерный траекторный аттрактор $\mathscr{U}$ семейства траекторных пространств $\left\{\mathscr{H}_{\sigma}^{+}: \sigma \in \Sigma\right\}$ системы (6.19), (6.20).

ДокАЗАТЕльство. Для доказательства достаточно построить полуаттрактор семейства пространств $\left\{\mathscr{H}_{\sigma}^{+}: \sigma \in \Sigma\right\}$. Заметим, что из свойства (iii) определения 6.11 следует, что каждая траектория $(v, \tau)$ каждого из пространств $\mathscr{H}_{\sigma}^{+}$ удовлетворяет неравенству

$$
\begin{aligned}
& \|v\|_{L_{\infty}(t, t+1 ; H)}^{2}+\|\tau\|_{L_{\infty}\left(t, t+1 ; L_{2}\right)}^{2}+\|v\|_{L_{2}(t, t+1 ; V)}^{2} \\
& \quad \leqslant C^{2}\left(1+e^{-2 \gamma t}\left(\|v\|_{L_{\infty}\left(\mathbb{R}_{+} ; H\right)}^{2}+\|\tau\|_{L_{\infty}\left(\mathbb{R}_{+} ; L_{2}\right)}^{2}\right)\right)
\end{aligned}
$$

с некоторой постоянной $C>0$, не зависящей ни от пространства, ни от траектории. Рассмотрим множество $P \subset C\left(\mathbb{R}_{+} ; E_{0}\right) \cap L_{\infty}\left(\mathbb{R}_{+} ; E\right)$, состоящее из пар $(v, \tau) \in C\left(\mathbb{R}_{+} ; E_{0}\right) \cap L_{\infty}\left(\mathbb{R}_{+} ; E\right)$, для которых при всех $t \geqslant 0$ выполняются неравенства

$$
\|v\|_{L_{\infty}(t, t+1 ; H)}^{2}+\|\tau\|_{L_{\infty}\left(t, t+1 ; L_{2}\right)}^{2}+\|v\|_{L_{2}(t, t+1 ; V)}^{2} \leqslant 2 C^{2},
$$




$$
\begin{aligned}
& \left\|v^{\prime}\right\|_{L_{4 / 3}\left(t, t+1 ; V^{*}\right)}+\left\|\tau^{\prime}\right\|_{L_{2}\left(t, t+1 ; H^{-2}\right)} \\
& \quad \leqslant \mathscr{M}\left(\|v\|_{L_{\infty}(t, t+1 ; H)},\|v\|_{L_{2}(t, t+1 ; V)},\|\tau\|_{L_{\infty}\left(t, t+1 ; L_{2}\right)},\|f\|_{\mathscr{X}}, 1\right),
\end{aligned}
$$

где $\mathscr{M}$ - функция из леммы 6.1 .

Ясно, что Т $(t) P \subset P(t \geqslant 0)$. Из неравенства (6.30) следует, что множество $P$ ограничено в $L_{\infty}\left(\mathbb{R}_{+} ; H\right) \times L_{\infty}\left(\mathbb{R}_{+} ; L_{2}\right)=E$. Из неравенств $(6.30)$ и $(6.31)$ следует, что для любого $T>0$ нормы $\left\|v^{\prime}\right\|_{L_{4 / 3}\left(0, T ; V^{*}\right)}$ и $\left\|\tau^{\prime}\right\|_{L_{2}\left(0, T ; H^{-2}\right)}$ ограничены в совокупности для $(v, \tau) \in P$. Следовательно, множество $P$ относительно компактно в $E_{0}$.

Легко видеть, что $P$ является поглощающим множеством для $\mathscr{H}^{+}$. Действительно, пусть множество $B \subset \mathscr{H}^{+}$ограничено в $E$ и

$$
\|v\|_{L_{\infty}\left(\mathbb{R}_{+} ; H\right)} \leqslant R, \quad\|\tau\|_{L_{\infty}\left(\mathbb{R}_{+} ; L_{2}\right)} \leqslant R \quad \text { для }(v, \tau) \subset B .
$$

Пусть число $t_{B} \geqslant 0$ таково, что $2 R^{2} e^{-2 \gamma t_{B}} \leqslant 1$. Согласно неравенству (6.29) для $h \geqslant t_{B}$ и $t \geqslant 0$ имеем

$$
\begin{aligned}
& \|\mathrm{T}(h) v\|_{L_{\infty}(t, t+1 ; H)}^{2}+\|\mathrm{T}(h) \tau\|_{L_{\infty}\left(t, t+1 ; L_{2}\right)}^{2}+\|\mathrm{T}(h) v\|_{L_{2}(t, t+1 ; V)}^{2} \\
& \quad=\|v\|_{L_{\infty}(t+h, t+h+1 ; H)}^{2}+\|\tau\|_{L_{\infty}\left(t+h, t+h+1 ; L_{2}\right)}^{2}+\|v\|_{L_{2}(t+h, t+h+1 ; V)}^{2} \\
& \quad \leqslant C^{2}\left(1+e^{-2 \gamma t+h}\left(\|v\|_{L_{\infty}\left(\mathbb{R}_{+} ; H\right)}^{2}+\|\tau\|_{L_{\infty}\left(\mathbb{R}_{+} ; L_{2}\right)}^{2}\right)\right) \\
& \quad \leqslant C^{2}\left(1+2 R^{2} e^{-2 \gamma t_{B}}\right) \leqslant 2 C^{2}
\end{aligned}
$$

и неравенство (6.30) выполняется для функции $\mathrm{T}(h)(v, \tau)$ при всех $t \geqslant 0$. Также для этой функции выполняется неравенство (6.31), так как оно выполняется для $(v, \tau)$ при всех $t \geqslant 0$. Следовательно, $\mathrm{T}(h) B \subset P$ при $h \geqslant t_{B}$, и $P-$ поглощающее множество.

Согласно лемме 4.2 замыкание $\bar{P}$ множества $P$ в пространстве $C\left(\mathbb{R}_{+} ; E_{0}\right)$ является траекторным полуаттрактором объединенного пространства траекторий $\mathscr{H}_{\Sigma}^{+}$, т. е. семейства $\left\{\mathscr{H}_{\sigma}^{+}: \sigma \in \Sigma\right\}$. По общей теореме 6.1 отсюда следует существование минимального равномерного траекторного аттрактора. Теорема 6.5 доказана.

Теорема 6.6 [17]. В условиях теоремы 6.5 в пространстве $\mathrm{H} \times \mathrm{L}_{2}$ существует равномерный глобальный аттрактор семейства траекторных пространств $\left\{\mathscr{H}_{\sigma}^{+}: \sigma \in \Sigma\right\}$ (в топологии $V_{\alpha}^{*} \times H^{-\alpha}$ ) системы (6.19), (6.20).

ДокАзАтЕльство. Согласно теореме 6.1 для существования равномерного глобального аттрактора семейства пространства траекторий достаточно существования минимального равномерного траекторного аттрактора. Однако существование последнего устанавливается теоремой 6.5. Теорема 6.6 доказана.

\section{Список литературы}

[1] О.А. Ладыженская, "О динамической системе, порождаемой уравнениями Навье-Стокса", Краевье задачи математической физики и смежные вопросы теории функиий. 6, Зап. науч. сем. ЛОМИ, 27, Изд-во «Наука», Ленингр. отд., Л., 1972, 91-115. 
[2] О.А. Ладыженская, "О нахождении минимальных глобальных аттракторов для уравнений Навье-Стокса и других уравнений с частными производными”, УМН, 42:6(258) (1987), 25-60; англ. пер.: О. A. Ladyzhenskaya, "On the determination of minimal global attractors for the Navier-Stokes and other partial differential equations", Russian Math. Surveys, 42:6 (1987), 27-73.

[3] V. V. Chepyzhov, M. I. Vishik, "Trajectory attractors for evolution equations", C. $R$. Acad. Sci. Paris Sér. I Math., 321:10 (1995), 1309-1314.

[4] V. V. Chepyzhov, M. I. Vishik, "Evolution equations and their trajectory attractors", J. Math. Pures Appl. (9), 76:10 (1997), 913-964.

[5] G. Sell, "Global attractors for the three-dimensional Navier-Stokes equations", J. Dynam. Differential Equations, 8:1 (1996), 1-33.

[6] V.V. Chepyzhov, M.I. Vishik, Attractors for equations of mathematical physics, Amer. Math. Soc. Colloq. Publ., 49, Amer. Math. Soc., Providence, RI, 2002, xii+363 pp.

[7] М.И. Вишик, В.В. Чепыжов, "Траекторные аттракторы уравнений математической физики”, УМH, 66:4(400) (2011), 3-102; англ. пер.: M.I. Vishik, V.V. Chepyzhov, "Trajectory attractors of equations of mathematical physics", Russian Math. Surveys, 66:4 (2011), 637-731.

[8] G. R. Sell, Y. You, Dynamics of evolutionary equations, Appl. Math. Sci., 143, Springer-Verlag, New York, 2002, xiv+670 pp.

[9] А.В. Бабин, М.И. Вишик, Аттракторы эволющионных уравнений, Наука, М., 1989, 296 с.; англ. пер.: A. V. Babin, M. I. Vishik, Attractors of evolution equations, Stud. Math. Appl., 25, North-Holland Publishing Co., Amsterdam, 1992, x+532 pp.

[10] В. В. Чепыжов, "О равномерных аттракторах динамических процессов и неавтономных уравнений математической физики”, УМН, 68:2(410) (2013), 159-196; англ. пер.: V.V. Chepyzhov, "Uniform attractors of dynamical processes and non-autonomous equations of mathematical physics", Russian Math. Surveys, 68:2 (2013), 349-382.

[11] Д. А. Воротников, В. Г. Звягин, "О траекторных и глобальных аттракторах для уравнений движения вязкоупругой среды”, УМН, 61:2(368) (2006), 161-162; англ. пер.: D. A. Vorotnikov, V. G. Zvyagin, "On the trajectory and global attractors for the equations of motion of a visco-elastic medium", Russian Math. Surveys, 61:2 (2006), 368-370.

[12] D. A. Vorotnikov, V. G. Zvyagin, "Uniform attractors for non-automous motion equations of viscoelastic medium", J. Math. Anal. Appl., 325:1 (2007), 438-458.

[13] D. A. Vorotnikov, V. G. Zvyagin, "Trajectory and global attractors of the boundary value problem for autonomous motion equations of viscoelastic medium", J. Math. Fluid Mech., 10:1 (2008), 19-44.

[14] V. G. Zvyagin, D. A. Vorotnikov, Topological approximation methods for evolutionary problems of nonlinear hydrodynamics, De Gruyter Ser. Nonlinear Anal. Appl., 12, Walter de Gruyter \& Co., Berlin, 2008, xii+230 pp.

[15] С. К. Кондратьев, “Об аттракторах модели движения слабо концентрированных водных растворов полимеров", Вестн. Воронеж. гос. ун-та. Сер. Физ. Матем., 2010, № 1, 117-138.

[16] В. Г. Звягин, С. К. Кондратьев, “Аттракторы слабых решений регуляризованной системы уравнений движения жидких сред с памятью”, Изв. вузов. Матем., 2011, № 8, 86-89; англ. пер.: V. G. Zvyagin, S. K. Kondrat'ev, "Attractors of weak solutions to a regularized system of motion equations for fluids with memory", Russian Math. (Iz. VUZ), 55:8 (2011), 75-77. 
[17] V. G. Zvyagin, D. A. Vorotnikov, "Approximating-topological methods in some problems of hydrodynamics", J. Fixed Point Theory Appl., 3:1 (2008), 23-49.

[18] R. Temam, Infinite-dimensional dynamical systems in mechanics and physics, Appl. Math. Sci., 68, Springer-Verlag, New York, 1988, xvi+500 pp.

[19] В. Г. Звягин, В. Т. Дмитриенко, Аппроксимачионно-топологический подход к исследованию задач гидродинамики. Система Навъе-Стокса, Едиториал УРСС, M., 2004, 112 c.

[20] О.А. Ладыженская, Математические вопросы динамики вязкой несжимаемой жидкости, 2-е изд., Наука, М., 1970, 288 с.; англ. пер. 1-го изд.: О. А. Ladyzhenskaya, The mathematical theory of viscous incompressible flow, 2nd English ed., rev. and enlarged, Math. Appl., 2, Gordon and Breach Science Publishers, New York-London-Paris, 1969, xviii+224 pp.

[21] А. В. Бабин, М. И. Вишик, “Аттракторы системы Навье-Стокса и параболических уравнений и оценка их размерности", Краевые задачи математической физики и смежные вопросы теории функиий. 14, Зап. науч. сем. ЛОМИ, 115, Изд-во «Наука», Ленингр. отд., Л., 1982, 3-15; англ. пер.: А. V. Babin, M. I. Vishik, "Attractors of Navier-Stokes systems and of parabolic equations, and estimates for their dimensions", J. Soviet Math., 28:5 (1985), 619-627.

[22] М.И. Вишик, В. В. Чепыжов, “Траекторный и глобальный аттракторы 3D системы Навье-Стокса", Матем. заметки, 71:2 (2002), 194-213; англ. пер.: M. I. Vishik, V. V. Chepyzhov, "Trajectory and global attractors of three-dimensional Navier-Stokes systems", Math. Notes, 71:2 (2002), 177-193.

[23] А.В. Фурсиков, Оптимальное управление распределенными системами. Теория и приложения, Научная книга, Новосибирск, 1999, 352 с.; англ. пер.: A. V. Fursikov, Optimal control of distributed systems. Theory and applications, Transl. Math. Monogr., 187, Amer. Math. Soc., Providence, RI, 2000, xiv+305 pp.

[24] М. Рейнер, Реология, Физматгиз, М., 1965, 224 с.; пер. с англ.: M. Reiner, "Rheology", Handbuch der Physik, v. 6: Elastizität und Plastizität, ed. S. Flügge, Springer-Verlag, Berlin-Göttingen-Heidelberg, 1958, 434-550.

[25] D. A. Vorotnikov, V. G. Zvyagin, "On the existence of weak solutions for the initialboundary value problem in the Jeffreys model of motion of a viscoelastic medium", Abstr. Appl. Anal., 2004:10 (2004), 815-829.

[26] В. А. Павловский, "К вопросу о теоретическом описании слабых водных растворов полимеров", Докл. АН СССР, 200:4 (1971), 809-812.

[27] В. Б. Амфилохиев, Я. И. Войткунский, Н. П. Мазаева, Я. С. Ходорковский, "Течения полимерных растворов при наличии конвективных ускорений”, Тр. Ленингр. кораблестр. ин-та, 1975, № 96, 3-9.

[28] В. Г. Звягин, М. В. Турбин, Математические вопросы гидродинамики вязкоупругих сред, КРАСАНД, М., 2012, 416 с.

[29] В. Г. Звягин, М. В. Турбин, "Исследование начально-краевых задач для математических моделей движения жидкостей Кельвина-Фойгта", Гидродинамика, СМФН, 31, РУДН, М., 2009, 3-144; англ. пер.: V. G. Zvyagin, M. V. Turbin, "The study of initial-boundary value problems for mathematical models of the motion of Kelvin-Voigt fluids", J. Math. Sci. (N. Y.), 168:2 (2010), 157-308.

[30] В. Т. Дмитриенко, В.Г. Звягин, "Конструкции оператора регуляризации в моделях движения вязкоупругих сред”, Вестн. Воронеж. гос. ун-та. Сер. Физ. Матем., 2004, № 2, 148-153. 
[31] В. Г. Звягин, В. Т. Дмитриенко, "О слабых решениях начально-краевой задачи для уравнения движения вязкоупругой жидкости”, Докл. РАН, 380:3 (2001), 308-311; англ. пер.: V. G. Zvyagin, V. T. Dmitrienko, "On weak solutions of an initial-boundary value problem for the equation of motion of a viscoelastic fluid", Dokl. Math., 64:2 (2001), 190-193.

[32] В. Г. Звягин, В. Т. Дмитриенко, "О слабых решениях регуляризованной модели вязкоупругой жидкости”, Дифферени. уравнения, 38:12 (2002), 1633-1645; англ. пер.: V. G. Zvyagin, V.T. Dmitrienko, "On weak solutions of a regularized model of a viscoelastic fluid", Differ. Equ., 38:12 (2002), 1731-1744.

[33] В.Г. Звягин, С.К. Кондратьев, “Аттракторы слабых решений регуляризованной системы уравнений движения жидких сред с памятью", Матем. сб., 203:11 (2012), 83-104; англ. пер.: V. G. Zvyagin, S. K. Kondrat'ev, "Attractors of weak solutions to the regularized system of equations of motion of fluid media with memory", Sb. Math., 203:11 (2012), 1611-1630.

[34] A. N. Carvalho, J.A. Langa, J.C. Robinson, Attractors for infinite-dimensional non-autonomous dynamical systems, Appl. Math. Sci., 182, Springer, New York, 2013, xxxvi+409 pp.

\section{Виктор Григорьевич Звягин}

(Viktor G. Zvyagin)

Воронежский государственный университет

E-mail: zvg@math.vsu.ru

\section{Станислав Константинович Кондратьев \\ (Stanislav K. Kondrat'ev)}

Воронежский государственный университет

E-mail: kondratev@math.vsu.ru
Поступила в редакцию 22.06 .2014 